\title{
Clinical trial discrimination of physical function instruments for psoriatic arthritis: a
}

systematic review

Ying Ying Leung ${ }^{1}$, Richard Holland ${ }^{2}$ Ashish J Mathew ${ }^{3,4,5}$, Chris Lindsay ${ }^{6}$, Niti Goel ${ }^{7}$, Alexis Odgie ${ }^{8}$, Ana-Maria Orbai $^{9}$, Pil Hojgaard ${ }^{10}$, Jeffrey Chau ${ }^{11}$, Laura C Coates ${ }^{12}$, Vibeke Strand $^{13}$, Dafna D Gladman ${ }^{14}$, Robin Christensen ${ }^{15}$, William Tillett ${ }^{16}$, Philip Mease ${ }^{17}$

1. Singapore General Hospital, Duke-NUS Medical School, Singapore

2. Concord Repatriation General Hospital, Sydney, Australia

3. Centre for Prognosis Studies in Rheumatic Diseases, Division of Rheumatology, Department of Medicine, University of Toronto, Toronto, Ontario Canada

4. Department of Clinical Immunology \& Rheumatology, Christian Medical College, Vellore, India

5. The Copenhagen Center for Arthritis Research, Rigshospitalet Glostrup, University of Copenhagen, Copenhagen, Denmark

6. Patient research partner, Prosper, Texas USA

7. Patient Research Partner, Adjunct Assistant Professor, Duke University School of Medicine, Durham, North Carolina, USA

8. Medicine and Epidemiology, Perelman School of Medicine, University of Pennsylvania, Philadelphia, Pennsylvania, USA

9. Division of Rheumatology, Johns Hopkins University School of Medicine, Baltimore, Maryland, USA

10. Copenhagen Lupus and Vasculitis Clinic, Center for Rheumatology and Spine Diseases, Copenhagen University Hospital, Rigshospitalet, Copenhagen, Denmark.

11. Patient Research Partner 
12. National Institute for Health Research Clinician Scientist, Nuffield Department of Orthopaedics, Rheumatology and Musculoskeletal Sciences, University of Oxford, Oxford, United Kingdom

13. Division of Immunology/Rheumatology, Stanford University School of Medicine, Palo Alto, California, USA

14. Medicine, University of Toronto, Senior Scientist, Krembil Research Institute, Director, Psoriatic Arthritis Program, University Health Network, Toronto Western Hospital, Toronto, Ontario, Canada

15. Musculoskeletal Statistics Unit, The Parker Institute, Bispebjerg and Frederiksberg Hospital, University of Copenhagen, Copenhagen \& Research Unit of Rheumatology, Department of Clinical Research, University of Southern Denmark, Odense University Hospital, Denmark.

16. Royal National Hospital for Rheumatic Diseases, University of Bath, Bath, United Kingdom

17. Rheumatology Research, Swedish Medical Center and University of Washington School of Medicine, Seattle, Washington, USA

Correspondence to: Ying-Ying Leung, MD; Department of Rheumatology and Immunology, Singapore General Hospital, The Academia, level 4, 20 College Road, Singapore 169856, Contact No.: +65 63265276, Fax no.: +65 62203321, E-mail: katyccc@hotmail.com

Key words: Systematic Review, psychometric, physical function, psoriatic arthritis 


\section{ABSTRACT}

Objectives. Physical function (PF) is a core domain to be measured in randomized controlled trials (RCTs) of psoriatic arthritis (PsA), yet the discriminative performance of patient reported outcome measures (PROMs) for PF in RCTs has not been evaluated systematically. In this systematic review, we aimed to evaluate the clinical trial discrimination of PF-PROMs in PsA RCTs.

Methods. We searched PubMed and Scopus databases in English to identify all original RCTs on biological and targeted synthetic disease modifying anti-rheumatic drugs (DMARDs) conducted in PsA. We assessed quality in each article using the OMERACT good method checklist. Effect sizes (ES) for the PF-PROMs were calculated and appraised using a priori hypotheses. Evidence supporting clinical trial discrimination for each PFPROM was summarized to derive recommendations.

Results. 35 articles from 31 RCTs were included. Four PF-PROMs had data for evaluation: HAQ-Disability Index (DI), HAQ-Spondyloarthritis (S), and Short Form 36-item Health Survey Physical Component Summary (SF-36 PCS) and Physical Functioning domain (SF$36 \mathrm{PF})$. As anticipated, higher ES values were observed for intervention groups than the control groups. Across all studies, for HAQ-DI, the median ES were -0.73 and -0.24 for intervention and control groups, respectively. Whereas for SF-36 PCS, the median ES were 0.77 and 0.23 . for intervention and control groups, respectively.

Conclusion. Clinical trial discrimination was supported for HAQ-DI and SF-36 PCS in PsA with low risk of bias; and for SF-36 PF and HAQ-S with some caution. More studies are required for HAQ-S.

Keywords: Psoriatic arthritis, physical function, responsiveness, clinical trials, psychometric 


\section{Clinical significance}

- This is the first paper that systematically appraised the clinical trial discrimination properties for PF-PROMs in PsA.

- Data for appraisal of clinical trial discrimination were available for only four PFPROMs (HAQ-DI, HAQ-S, SF-36 PCS and SF-36 PF).

- The HAQ-DI and SF-36 PCS demonstrated clinical trial discrimination with low risk of bias.

- Clinical trial discrimination with SF-36 PF and HAQ-S are supported with caution. More studies are needed for SF-36 PF and HAQ-S. 


\section{INTRODUCTION}

Psoriatic arthritis (PsA) is a chronic inflammatory disease involving peripheral arthritis, enthesitis, dactylitis, spondylitis, psoriasis and nail disease (1). It has a profound impact on patients' physical, psychological and social well-being. The inflammation of axial and peripheral joints as well as entheses causes pain as well as joint erosion and destruction over the longer term (2). Both the acute inflammation and joint damage from PsA cause loss of physical function (PF) and disability (3). Physical function is a key concern from numerous qualitative studies among PsA patients $(4,5)$, and it is recognized as one of the core domains to be measured in every randomized controlled trial (RCT) and longitudinal observational study (6).

There have been several patient-reported outcome measures (PROMs) that assess PF in PsA (7), the most commonly used in RCTs of PsA being the Health Assessment Questionnaire Disability Index (HAQ-DI) (8) and the physical functioning domain within the Medical Outcomes Study 36-item Short Form Survey (SF-36 PF) (9). A few additional ones have also been validated and evaluated for use in PsA (10). The discriminative performance of these PF-PROMs in RCTs have not been evaluated systematically. In this systematic review, we aimed to evaluate the clinical trial discrimination properties of PF-PROMs in RCTs of PsA. The data derived from this study contribute to the concerted effort of the Group for Research And Assessment of Psoriasis and Psoriatic Arthritis (GRAPPA) Outcome Measures in Rheumatology (OMERACT) initiative to standardize an outcome measurement set for PsA (11). 


\section{METHODS}

The protocol of this systematic review was registered with the International Prospective Register of Systematic Reviews (PROSPERO) prior to initiation (CRD42019129557). The report of this systematic review adheres to the Preferred Reporting Items for Systematic reviews and Meta-Analysis (PRISMA statement) (12).

\subsection{Literature search and eligibility of articles}

We performed a database search through 21 March 2019 using PubMed and Scopus. The search aimed to identify all original full text articles of RCTs conducted in PsA with published data in the English language. The detail of search terms is summarized in Appendices (Table A.1). We included articles on RCTs conducted in PsA only. If the RCT was conducted in a mixed arthritis population, we only included those with separate subgroup analyses in PsA. We excluded RCTs that were not double blind in study design. For the purpose of deriving responsiveness data for the PF-PROMs, we limited the review to RCTs involving biological (b) disease modifying anti-rheumatic drugs (DMARDs) and targeted synthetic (ts)DMARDs. For RCTs with multiple publications, only one article from each unique RCT was included, unless a publication provided new information, such as subgroup analyses. We also excluded articles that did not report data for PF-PROMs.

\subsection{Selection of articles}

One researcher (YYL) removed duplicates from the searches in the two databases. Two researchers (YYL, RH) independently screened the titles, abstracts and full text (if appropriate) for eligibility. Disputes were resolved by consensus of the two researchers and a third researcher, if needed (AMO). Additional studies identified by the co-authors were 
considered for inclusion. The stepwise eligibility and inclusion of articles are summarized in a flow diagram (Figure 1).

\subsection{Extraction of study characteristics and description of PROMs}

YYL and AO independently extracted data on the characteristics of the studies including gender, disease duration of participants, proportion of participants taking methotrexate (MTX) and previous exposure (13) to tumor necrosis factor inhibitors (TNFi), active interventions, comparators, primary outcomes and their time points. Researchers worked in pairs (YYL and RH; YYL and AJM; YYL and NG; YYL and CL) to extract data independently for PF-PROMs at baseline and the time point of assessment of the primary outcome (or end of the blinded controlled period). These assessment time points were chosen as that they represented the time to measure responsiveness of the PF-PROMs for the active interventional from the control groups, as most RCTs offered cross-over to the active intervention after the primary end point. One researcher (YYL) calculated the effect sizes (ESs) for the PF-PROMs based on published data and online supplementary materials if available. Effect sizes of PF-PROMs comparing the active interventional and control groups were evaluated using the following methods wherever data were available (3): 1) Glass's effect size $\left(E S_{1}\right)$ : calculated by change scores divided by standard deviations (SDs) of baseline scores; 2) Cohen's effect size ( $\left.\mathrm{ES}_{2}\right)$, calculated by change scores divided by pooled SDs; and 3) standardized response means (SRMs), calculated by change scores divided by SDs of the change scores when no comparator group was available.

For papers that provided the standard error (SE) for individual groups, SD was

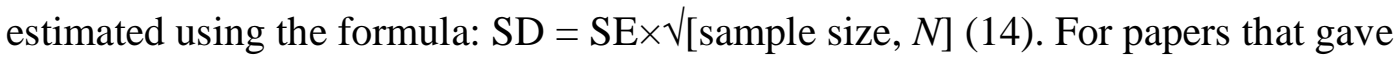
information on median and interquartile ranges, means (SD) were calculated using an estimation formula suggested by Wan et al (13). 


\subsection{Quality Assessment}

We assessed the clinical trial discrimination of PF-PROMs in each article using the OMERACT good method checklist (15). Two researchers in groups (YYL and RH; YYL and AJM; YYL and NG; YYL and CL) appraised each instrument for the following categories using (+) yes, good methods; (+/-) some cautions; or (-) no, not achieved:

1. Was the time interval between testing stated and appropriate?

2. Was there a proportion of participants expected to change in one or both groups?

3. Were hypotheses regarding the anticipated mean differences in change scores between subgroups described a priori?

4. Were the statistical methods adequate for the hypotheses tested?

5. Otherwise good methods? (free of any other important flaws)

The two-researcher groups independently assessed a final decision for each article rated as 1) Green, likely low risk of bias; 2) Amber, some cautions but can be used as evidence and 3) Red, not to use as evidence. Consensus between the two researchers in the group was sought and disputes were resolved with a third member of the team, if necessary.

\subsection{Appraisal of effect sizes using a priori hypotheses}

The calculated ESs of each article were appraised with the following a priori hypotheses. These hypotheses were developed based on general knowledge of the therapeutic efficacy of various treatments:

1. At the assessment time point (primary outcome or end of blinded controlled period), patients treated with bDMARDs have significant changes in PF-PROMs, whereas 
patients receiving the control intervention do not (except for alefacept [ALC] and clazakizumab [CLZ] where no significant differences were expected).

2. PF-PROM change scores among patients treated with bDMARDs are significantly better than those of the control group.

3. Within an individual trial, the ESs of PF-PROMs are higher in the bDMARD groups compared to the control groups that were treated with placebo, MTX or conventional synthetic (cs) DMARDs, but do not differ significantly from groups treated with different bDMARD doses (or groups treated with TNFi as a comparator).

4. If data for subgroup analyses are available, the ESs of change scores of the PF-PROM are higher in TNFi-naïve versus TNFi-exposed subgroups.

We assessed whether the hypotheses were satisfied using symbols of (+), when hypothesis satisfied; (+/-), if partially satisfied; or (-), not satisfied. As per OMERACT Filter 2.1 guidelines (15), we synthesized and summarized the overall evidence to support clinical trial discrimination for each PF-PROM in a Summary of Measurement Properties evidence (SOMP) table.

\subsection{Evidence synthesis}

In the SOMP table, we presented the number of articles available for measurement properties and evidence synthesis. For each article, color coding of Green/Amber/Red indicated the quality assessment and (+), (+/-) or (-) was given to indicate the a priori hypotheses were satisfied, partially satisfied or not satisfied.

For each PF-PROM, a final rating for clinical trial discrimination was given as GREEN (Good to go)/AMBER (Some concern but good to go)/RED (Stop, not to use this for 
evidence synthesis), or WHITE for no information available. This final rating follows the OMERACT Filter 2.1 recommendation for the quality assessment of articles, the number of good quality articles available, the consistency across articles and the PF-PROM performance in RCT discrimination (16). A PF-PROM that was supported by at least two good quality articles showing consistent findings with adequate performance in RCT discrimination would achieve GREEN. A PROM having at least two articles, but inconsistent findings; or having only one article with inadequate performance would be given a RED. In all other situations, a final rating of AMBER would be given.

\section{RESULTS}

\subsection{Literature search results}

Our literature search identified a total of 676 articles. After removal of duplicates, 608 articles remained; three articles were identified through cross-referencing check. There were 439 articles excluded for the following reasons: not RCTs (344 articles), open-label trials (10 articles), not in PsA (65 articles), mixed population of arthritis without separate data reported for PsA (18 articles) and trial protocols (2 articles). We retained 172 articles for full text review as RCTs of PsA. We excluded 48 articles as trials for interventions other than bDMARDs or tsDMARDs and 70 articles as secondary analyses of RCTs in PsA. From the 45 articles of 41 unique RCTs in PsA for bDMARDs and tsDMARDs, we further excluded 10 articles without reported data on PF-PROMs. Finally, 35 relevant articles from 31 RCTs were retained for qualitative assessment (Figure 1).

The characteristics of the included articles are summarized in Table 1 . There were 10 articles that did not report data on the PF domains. Among them, one article was from a phase $1 \mathrm{~b}$ and three articles were from phase 2 trials. Among the six phase $3 \mathrm{RCT}$ s that did not 
include PF data, two were small pilot "proof of concept" studies with a molecular and tissue focus, three were RCTs with a primary focus on psoriasis, while one was in PsA.

Among the included 31 RCTs, PF was evaluated using four PF-PROMs, including the HAQ-DI (8), HAQ-Spondyloarthritis (HAQ-S) (17), Physical Component Summary Score of the SF-36 (SF-36 PCS) (9), and the SF-36 PF (9). New potential PF-PROMs shortlisted by GRAPPA for further evaluation (18), including the multidimensional HAQ (MDHAQ) and the Patient-Reported Outcomes Measurement Information System (PROMIS)-Short Form Physical Function 10a (PROMIS-PF10a), were not utilized in the RCTs included in this review.

\subsection{Clinical trial discrimination for PF-PROMs}

\subsubsection{HAQ-DI}

Results were reported for HAQ-DI in 31 articles from 30 unique RCTs. The ESs calculated for both interventional and comparator groups are shown in Table 2. Effect sizes for HAQ-DI could not be calculated from two published articles (Appendices, Table A.2) and data were not used for evidence synthesis. Quality assessment using the OMERACT good method checklist was generally affirmative (Appendices, Table A.3). Minor concerns for quality were noted in 10 articles, predominantly resulting from not clearly stating expected change scores in HAQ-DI (although most expected changes were implied), estimating ESs from median/ interquartile ranges or using percentage changes that raised concern for introduction of minor errors. There were 29 articles included for evidence synthesis as shown in the SOMP table (Table 6). Results aligned with the a priori hypotheses that statistically significant change scores were reported in interventional but not in control groups with higher ESs for interventional compared to control groups (Table 2). The median (interquartile ranges, IQR) 
ES were $-0.73(-0.84,-0.62)$ and $-0.24(-0.39,-0.09)$ for intervention and control groups, respectively. Three articles included subgroup analyses, where one demonstrated higher ESs for HAQ-DI in TNFi-naïve compared with TNFi-exposed groups (19), while two articles did not $(20,21)$. The working group recommended a final rating of GREEN to support HAQ-DI for clinical trial discrimination in PsA, indicating a low risk of bias and results aligned with hypotheses.

\subsubsection{HAQ-S}

There was only one article that reported results for HAQ-S (22), showing higher ESs for the ABT-122 and adalimumab groups compared to control which aligned with the $a$ priori hypotheses (Table 3). Detail of the quality assessment is given in Appendices (Table A.4) The working group recommended AMBER for HAQ-S (Table 6), recognizing that more data are required for better evidence synthesis.

\section{$\underline{\text { 3.2.3 SF-36 PCS }}$}

The SF-36 was included in 26 out of 31 (83.9\%) RCTs in PsA. All 26 RCTs reported SF-36 PCS results, but only four reported on SF-36 PF. Of the articles included for evidence synthesis for SF-36 PCS, one did not pass the OMERACT checklist for evidence synthesis (23) for two reasons. It failed to include adequate data for ES calculations. Compared to control groups, statistically significant differences in change scores were only evident with the higher dose but not the lower dose interventional groups (Table 4). For the 25 articles included for evidence synthesis for SF-36 PCS, most results aligned with the a priori hypotheses. The median (IQR) ES were $0.77(0.60,0.93)$ and $0.23(0.09,0.36)$ for intervention and control groups, respectively. Minor quality concerns were noted in seven 
articles, that were similar to those stated for HAQ-DI (Appendix, Table A.5). The working group recommended GREEN supporting clinical trial discrimination with SF-36 PCS.

\section{$\underline{\text { 3.2.4 SF-36 PF }}$}

Four articles reported data for SF-36 PF. Two did not have adequate data for ES estimation and were excluded from evidence synthesis (Table 5) (Appendix, Table A.6). Of the remaining two articles, both on tofacitinib $(24,25)$, higher ESs in the interventional groups were shown compared with the control (Table 5). However, due to other methodologic concerns, these two articles were judged to be Amber (+) in the quality assessment (Appendix, Table A.6). Based on the limited evidence, the working group recommended AMBER for clinical trial discrimination with SF-36 PF (Table 6).

\section{DISCUSSION}

In this systematic review, we summarized the clinical trial discrimination of the available PF-PROMs in PsA. Of the 41 unique RCTs in PsA with bDMARDs or tsDMARDs, 31 (75.6\%) reported results of at least one measurement of the PF domain. This is the first paper to our knowledge that systematically appraised the clinical trial discrimination properties for PF-PROMs. Numerous instruments are available for assessing PF in PsA (7, 10). However, data for appraisal of clinical trial discrimination was available for only four PF-PROMs (HAQ-DI, HAQ-S, SF-36 PCS and SF-36 PF). Most of the studies reported data for HAQ-DI and SF-36 PCS, while only four and one reported data for SF-36 PF and HAQ-S, respectively. This systematic review supports clinical trial discrimination with HAQ-DI and SF-36 PCS with low risk of bias. Clinical trial discrimination with SF-36 PF and HAQ-S are supported with caution due to the limited studies available for data synthesis. Further research 
is warranted. There are no data published to date to support clinical trial discrimination for potential PF-PROMs shortlisted by GRAPPA such as the MDHAQ and PROMIS-PF (18).

Physical function is one of the domains included in the core outcome set for reporting data in PsA RCTs and longitudinal observational studies (6). However, variations in reporting of outcomes in PsA clinical trials have been recognized and GRAPPA and OMERACT are committed to standardize the outcome measures with evidence (11). To appropriately evaluate the measurement properties of instruments using the OMERACT Filter 2.1, multiple measurement properties are considered, including domain match, feasibility, validity, testretest reliability, longitudinal construct validity, clinical trial discrimination and threshold of meaning (15). In this study, we aimed to evaluate only the clinical trial discrimination of PF-PROMs. This represents an intermediate but important step in standardizing the outcome measurement set for PF in PsA.

The strength of the current work is the combined effort of investigators and patient research partners. The investigators are familiar with the measurement of PF in PsA with representation from 4 continents. The patient research partners have participated in a wide range of research activities, including data extraction, quality assessment of articles and appraisal of ESs. We followed the methods recommended by the OMERACT Filter 2.1 methodology in quality assessment of each article, calculating the ESs using appropriate statistics to synthesize the evidence to support clinical trial discrimination (15). This was further strengthened by setting a priori hypotheses on the expected magnitude of ESs of intervention groups compared with control groups.

Some limitations are recognized. We limited the evidence from RCTs of bDMARDs and tsDMARDs and excluded RCTs evaluating solely csDMARDs to best represents RCTs in the 
modern era that include the appropriate core domains. We calculated the ESs of PF-PROMs from published data as access to the original dataset from the RCTs was not available. Where applicable, we used formulae to estimate the means (SD) from reported medians (IQR), which may result in variability. Nonetheless, this variability in ES estimations has been recognized and addressed in the quality assessment using the OMERACT good method checklist; and the detailed calculations are shown in the supplementary documents. Few definitions of estimated ESs $\left(\mathrm{ES}_{1}, \mathrm{ES}_{2}\right.$ or $\left.\mathrm{SRM}\right)$ were tabulated depending on availability of published data, which may not be directly comparable. This would attribute only to minor issues as all hypothesis testing was performed intra- rather than inter- trials using the same type of ESs. We have not compared the responsiveness of one PF-PROM with another, which may be an interesting topic to pursue. Finally, we used data from published RCTs in PsA which generally enroll patients with higher disease activity (and greater limitations in physical function) than the average patient seen in clinical practice to undergo potential highly efficacious treatment, and therefore higher responsiveness for the PF-PROMs are expected. It is thus unclear if these results would be directly applicable to pragmatic trials and longitudinal observational studies.

\section{CONCLUSION}

This systemic review supports clinical trial discrimination with HAQ-DI and SF-36 PCS with low risk of bias, while clinical trial discrimination with SF-36 PF and HAQ-S is supported with some caution. More studies are required for SF-36 PF and HAQ-S. 


\section{ACKNOWLEDGEMENTS AND FUNDING}

YYL is funded by the Clinician Scientist award of the National Medical Research Council, Singapore (NMRC/CSA-INV/0022/2017). The views expressed are those of the author(s) and not necessarily those of the NMRC. AMO is funded by the Jerome L. Greene Foundation Scholar Award, the Staurulakis Family Discovery Award, the Rheumatology Research Foundation, and the National Institutes of Health (NIH) through the Rheumatic Diseases Resource-based Core Center (P30-AR053503 Cores A and D, and P30-AR070254, Cores A and B). All statements in this report, including its findings and conclusions, are solely those of the authors and do not necessarily represent the views of the NIH, the National Institute of Arthritis Musculoskeletal and Skin Diseases (NIAMS), or the Rheumatology Research Foundation (RRF). LCC is funded by a National Institute for Health Research Clinician Scientist award. The research was supported by the National Institute for Health Research (NIHR) Oxford Biomedical Research Centre (BRC). The views expressed are those of the author(s) and not necessarily those of the NHS, the NIHR, or the Department of Health. AO is funded by the Rheumatology Research Foundation and NIH/NIAMS R01 AR072363. The Parker Institute, Bispebjerg and Frederiksberg Hospital (PH and RC) is supported by a core grant from the Oak Foundation (OCAY-18-774-OFIL). 


\section{LIST OF ABBREVIATIONS}

ABT: abatacept;

ADA: adalimumab;

ALC: alefacept;

bDMARDs: biological disease modifying anti-rheumatic drugs;

BIW: twice a week;

BRO: brodalumab;

CI: confidence interval;

CLZ: clazakizumab;

csDMARDs: conventional synthetic disease modifying anti-rheumatic drugs;

CZP: certolizumab pegol;

ES: effect size;

ETN: etanercept;

EULAR: European League Against Rheumatism;

FIL: filgotinib;

GOL: golimumab;

GRAPPA: Group for Research and Assessment of Psoriasis and Psoriatic Arthritis

GUS: guselkumab;

HAQ-DI: Health Assessment Questionnaire - Disability Index;

HAQ-S: Health Assessment Questionnaire - Spondyloarthritis;

IFX: infliximab;

IL: interleukin;

IXE: ixekizumab;

IV: intravenous

IQR: interquartile range;

LS: least squares;

MDHAQ: multidimensional HAQ

MTX: methotrexate;

NA: not available;

NS: not significant;

OMERACT: Outcome Measures in Rheumatology

PASI: Psoriasis Area and Severity index;

PCB: placebo;

PCS: physical component summary of SF-36;

PF: physical functioning domain of SF-36;

PROM: patient reported outcome measure;

PROMIS: Patient-Reported Outcomes Measurement Information System;

PROSPERO: the International Prospective Register of Systematic Reviews;

PRISMA: Preferred Reporting Items for Systematic reviews and Meta-Analysis;

PsA: psoriatic arthritis;

Q2W: once every 2-week;

QW: once a week;

RCTs: randomized controlled trials

SD: standard deviation;

SE: standard error;

SEC: secukinumab;

SF-36: Medical Outcomes Study 36-item Short Form Survey;

TOF: tofacitinib; 
TNFi: tumor necrosis factor inhibitors;

tsDMARDs: targeted synthetic disease modifying anti-rheumatic drugs;

UST: ustekinumab;

vs.: versus. 


\section{TABLE AND FIGURE LEGEND}

Figure 1. Flow diagram for article selection

Table 1. Characteristics of included studies

Legend:

*early escape at Week 16;

Abbreviations: $\Delta$ : change; ACR: American College of Rheumatology Response criteria;

ABT: abatacept; ADA: adalimumab; ALC: alefacept; BIW: twice a week; BRO: brodalumab; CI: confidence interval; CLZ: clazakizumab; csDMARD: conventional synthetic disease modifying anti-rheumatic drug; CZP: certolizumab pegol; ETN: etanercept; EULAR: European League Against Rheumatism; FIL: filgotinib; GOL: golimumab; GUS:

guselkumab; HAQ-DI: Health Assessment Questionnaire - Disability index; HAQ-S: Health Assessment Questionnaire - Spondyloarthritis; IFX: infliximab; IXE: ixekizumab; IV: intravenous; IL=interleukin; IR=inadequate response; MTX: methotrexate; NA: not available; NS: not significant; PASI: Psoriasis Area and Severity Index; PCB: placebo; PCS: physical component summary of SF-36; PF: physical functioning domain of SF-36; PsA=psoriatic arthritis; PsARC: Psoriatic arthritis Response Criteria; QW: once a week; Q2W: once every 2 weeks; SD: standard deviation; SE: standard error; SEC: secukinumab; SF-36: Medical Outcomes Study 36-item Short Form Survey; SPARCC: Spondyloarthritis Research Consortium of Canada enthesitis index; TOF: tofacitinib; TNFi: tumor necrosis factor inhibitor; UST: ustekinumab; vs.: versus.

Table 2. Effect sizes estimation for studies reporting HAQ-DI Legend:

II SRM calculated using percentage change score and SD of percentage change; $\delta$ SRM for improvement, a negative value indicate deterioration; ¥ Effect sizes estimated based on mean and SD of change scores calculated from median and IQR from original publication; * early escape for patients with inadequate response in the PCB group to active treatment group at Week 16; ** option to switch TNFi at Week 24.

Abbreviations: $\Delta$ : change; ACR: American College of Rheumatology Response criteria; ABT: abatacept; ADA: adalimumab; ALC: alefacept; BIW: twice a week; BRO: brodalumab; CI: confidence interval; CLZ: clazakizumab; CZP: certolizumab pegol; ES2: Effect size 2 (the mean difference divided by the pooled standard deviation, i.e Cohen's d); ETN: etanercept; EULAR: European League Against Rheumatism; FIL: filgotinib; GOL: golimumab; GUS: guselkumab; HAQ-DI: Health Assessment Questionnaire - Disability Index; IFX: infliximab; IXE: ixekizumab; IV: intravenous; IQR: interquartile range; MTX: methotrexate; NA: not available; NS: not significant; PASI: Psoriasis Area and Severity Index; PCB: placebo; PsARC: Psoriatic arthritis Response Criteria; QW: once a week; Q2W: once every 2-week; SD: standard deviation; SE: standard error; SEC: secukinumab; SPARCC: Spondyloarthritis Research Consortium of Canada enthesitis index; SRM: Standardized response mean (mean difference divided by the standard deviation of the differences between baseline and assessment end point); TOF: tofacitinib; TNFi: tumor necrosis factor inhibitor; UST: ustekinumab; vs.: versus. 
Table 3. Effect sizes estimation for studies reported HAQ-S

Legend:

Abbreviations: ACR: American College of Rheumatology Response criteria; ADA: adalimumab; ES1: Effect size 1 (the mean difference divided by standard deviation of baseline score); HAQ-S: Health Assessment Questionnaire - Spondyloarthritis; PCB: placebo; vs.: versus.

Table 4. Effect sizes estimation for studies reported SF-36 PCS

Legend:

I SRM calculated using percentage change score and SD of percentage change; $\delta$ SRM for improvement, a negative value indicate deterioration; ¥ Effect sizes estimated based on mean and SD of change scores calculated from median and IQR from original publication; * early escape for patients with inadequate response in the PCB group to active treatment group at Week 16; ** option to switch TNFi at Week 24.

Abbreviations: $\Delta$ : change.ACR: American College of Rheumatology Response criteria; ABT: abatacept; ADA: adalimumab; ALC: alefacept; BIW: twice a week; BRO: brodalumab; CI: confidence interval; CLZ: clazakizumab; CZP: certolizumab pegol; ES2: Effect size 2 (the mean difference divided by the pooled standard deviation, i.e Cohen's d); ETN: etanercept; EULAR: European League Against Rheumatic Diseases; GOL: golimumab; GUS:

guselkumab; IFX: infliximab; IXE: ixekizumab; IV: intravenous; IQR: interquartile range; MTX: methotrexate; NA: not available; PASI: psoriasis area and severity index; PCB: placebo; PCS: physical component summary of SF-36; PF: physical functioning domain of SF-36; QW: once a week; Q2W: once every 2-week; SD: standard deviation; SE: standard error; SEC: secukinumab; SF-36: Medical Outcome Survey Short Form 36 items; SPARCC: Spondyloarthritis Research Consortium of Canada enthesitis index; SRM: Standardized response mean (mean difference divided by the standard deviation of the differences between baseline and assessment end point); TNFi: tumor necrosis factor inhibitor; UST:

ustekinumab; vs.: versus.

Table 5. Effect sizes estimation for studies reporting SF-36 PF.

Legend:

I SRM calculated using percentage change score and SD of percentage change; $\delta$ SRM for improvement, a negative value indicate deterioration; ¥ Effect sizes estimated based on mean and SD of change scores calculated from median and IQR from original publication; * early escape for patients with inadequate response in the control group to active treatment group at week 16; ** option to switch TNFi at Week 24.

Abbreviations: $\Delta$ : change; ACR: American College of Rheumatology Response criteria; ADA: adalimumab; ES2: Effect size 2 (the mean difference divided by the pooled standard deviation, i.e Cohen's d); FIL: filgotinib; HAQ-DI: Health Assessment Questionnaire Disability Index; IFX: infliximab; IQR: interquartile range; PCB: placebo; PF: physical functioning domain of SF-36; SD: standard deviation; SF-36: Medical Outcomes Study 36item Short Form Survey; SRM: Standardized response mean (mean difference divided by the standard deviation of the differences between baseline and assessment end point); TOF: tofacitinib; vs.: versus. 
Table 6. Summary of Measurement Properties Table for clinical trial discrimination Legend:

Color code in each box indicate study quality assessed by OMERACT good methods.

GREEN means "yes, likely low risk of bias"; AMBER means "some cautions but can be used as evidence" and RED means "No, don't use as evidence". WHITE (empty boxes), indicates absence of information on that property from that study. (+) indicates findings of the study had adequate performance of the instrument; (+/-) indicates equivocal performance; (-) indicates poor performance (less than adequate). 


\section{DECLARATION OF INTEREST}

This research did not receive any specific grant from funding agencies in the public, commercial, or not-for-profit sectors.

YYL is funded by the Clinician Scientist award of the National Medical Research Council, Singapore (NMRC/CSA-INV/0022/2017). AMO is funded by the Jerome L. Greene Foundation Scholar Award, the Staurulakis Family Discovery Award, the Rheumatology Research Foundation, and the National Institutes of Health (NIH) through the Rheumatic Diseases Resource-based Core Center (P30-AR053503 Cores A and D, and P30-AR070254, Cores A and B). PH and RC (The Parker Institute, Bispebjerg and Frederiksberg Hospital) is supported by a core grant from the Oak Foundation (OCAY-18-774-OFIL). LCC is funded by a National Institute for Health Research Clinician Scientist award, and the National Institute for Health Research (NIHR) Oxford Biomedical Research Centre (BRC). AO is funded by the Rheumatology Research Foundation and NIH/NIAMS R01 AR072363. All statements in this report, including its findings and conclusions, are solely those of the authors and do not necessarily represent the views of the funding agencies.

\section{AUTHOR CONTRIBUTIONS}

Ying Ying Leung: Conceptualization; Data curation; Formal analysis; Investigation; Methodology; Project administration; Resources; Software; Supervision; Validation; Visualization; Writing original draft; review \& editing. Richard Holland: Conceptualization; Data curation; Formal analysis; Investigation; Methodology; Project administration; Resources; Validation; Visualization; Writing - review \& editing. Ashish J Mathew: Data curation; Formal analysis; Investigation; Methodology; Resources; Validation; Writing - review \& editing. Chris Lindsay: Data curation; Formal analysis; 
Investigation; Methodology; Validation; Writing - review \& editing. Niti Goel: Data curation; Formal analysis; Investigation; Methodology; Validation; Visualization; Writing review \& editing. Alexis Odgie: Conceptualization; Data curation; Validation; Writing review \& editing. Ana-Maria Orbai: Conceptualization; Data curation; Methodology; Validation; Writing - review \& editing. Pil Hoejgaard: Methodology; Writing - review \& editing. Jeffrey Chau: Methodology; Writing - review \& editing. Laura C Coates: Methodology; Supervision; Writing - review \& editing. Vibeke Strand: Methodology; Supervision; Writing - review \& editing. Dafna D Gladman: Conceptualization; Methodology; Supervision; Writing - review \& editing. Robin Christensen: Formal analysis; Methodology; Resources; Supervision; Validation; Writing - review \& editing. William Tillett: Methodology; Supervision; Validation; Visualization; Writing - review \& editing. Philip Mease: Methodology; Supervision; Validation; Visualization; Writing review \& editing. 


\section{REFERNCES}

1. Gladman DD, Antoni C, Mease P, Clegg DO, Nash P. Psoriatic arthritis: epidemiology, clinical features, course, and outcome. Annals of the rheumatic diseases. 2005;64 Suppl 2:ii14-7.

2. Gladman DD, Farewell VT, Nadeau C. Clinical indicators of progression in psoriatic arthritis: multivariate relative risk model. The Journal of rheumatology. 1995;22(4):675-9.

3. Husted JA, Tom BD, Farewell VT, Schentag CT, Gladman DD. Description and prediction of physical functional disability in psoriatic arthritis: a longitudinal analysis using a Markov model approach. Arthritis and rheumatism. 2005;53(3):404-9.

4. Orbai AM, de Wit M, Mease PJ, Callis Duffin K, Elmamoun M, Tillett W, et al. Updating the Psoriatic Arthritis (PsA) Core Domain Set: A Report from the PsA Workshop at OMERACT 2016. The Journal of rheumatology. 2017;44(10):1522-8.

5. Dures E, Hewlett S, Lord J, Bowen C, McHugh N, Group PS, et al. Important Treatment Outcomes for Patients with Psoriatic Arthritis: A Multisite Qualitative Study. Patient. 2017;10(4):45562.

6. Orbai AM, de Wit M, Mease P, Shea JA, Gossec L, Leung YY, et al. International patient and physician consensus on a psoriatic arthritis core outcome set for clinical trials. Annals of the rheumatic diseases. 2017;76(4):673-80.

7. Mease P, Strand V, Gladman D. Functional impairment measurement in psoriatic arthritis: Importance and challenges. Seminars in arthritis and rheumatism. 2018;48(3):436-48.

8. Fries JF, Spitz P, Kraines RG, Holman HR. Measurement of patient outcome in arthritis. Arthritis and rheumatism. 1980;23(2):137-45.

9. Ware JE, Jr., Sherbourne CD. The MOS 36-item short-form health survey (SF-36). I. Conceptual framework and item selection. Med Care. 1992;30(6):473-83.

10. Hojgaard P, Klokker L, Orbai AM, Holmsted K, Bartels EM, Leung YY, et al. A systematic review of measurement properties of patient reported outcome measures in psoriatic arthritis: $A$ GRAPPA-OMERACT initiative. Seminars in arthritis and rheumatism. 2018;47(5):654-65.

11. Tillett W, Orbai AM, Ogdie A, Leung YY, Strand V, Gladman DD, et al. GRAPPA-OMERACT initiative to standardise outcomes in psoriatic arthritis clinical trials and longitudinal observational studies. Ann Rheum Dis. 2018;77(5):e23.

12. Liberati A, Altman DG, Tetzlaff J, Mulrow C, Gotzsche PC, loannidis JP, et al. The PRISMA statement for reporting systematic reviews and meta-analyses of studies that evaluate healthcare interventions: explanation and elaboration. BMJ (Clinical research ed). 2009;339:b2700.

13. Wan X, Wang W, Liu J, Tong T. Estimating the sample mean and standard deviation from the sample size, median, range and/or interquartile range. BMC Med Res Methodol. 2014;14:135.

14. Collaboration TC. Cochrane Handbook for Systematic Reviews of Interventions Version 5.1.0. In: Higgins JPT, Green S, editors. 2011.

15. Beaton DE, Maxwell L, Shea BJ, Wells GA, Boers M, Grosskleg S, et al. Instrument Selection Using the OMERACT Filter 2.1: The OMERACT Methodology. The Journal of rheumatology. 2019;46(8):1028-35.

16. Boers M, Kirwan JR, Tugwell P, Beaton D, Bingham III CO, Conaghan PG, et al. The OMERACT Handbook. 2017.

17. Daltroy LH, Larson MG, Roberts NW, Liang MH. A modification of the Health Assessment Questionnaire for the spondyloarthropathies. The Journal of rheumatology. 1990;17(7):946-50.

18. Leung YY, Orbai AM, Ogdie A, Hojgaard P, Holland R, Goel N, et al. Appraisal of candidate instruments for assessment of the physical function domain in patients with psoriatic arthritis. The Journal of rheumatology. 2020.

19. Kavanaugh A, McInnes IB, Mease PJ, Hall S, Chinoy H, Kivitz AJ, et al. Efficacy of Subcutaneous Secukinumab in Patients with Active Psoriatic Arthritis Stratified by Prior Tumor Necrosis Factor Inhibitor Use: Results from the Randomized Placebo-controlled FUTURE 2 Study. The Journal of rheumatology. 2016;43(9):1713-7. 
20. Ritchlin C, Rahman P, Kavanaugh A, McInnes IB, Puig L, Li S, et al. Efficacy and safety of the anti-IL-12/23 p40 monoclonal antibody, ustekinumab, in patients with active psoriatic arthritis despite conventional non-biological and biological anti-tumour necrosis factor therapy: 6-month and 1-year results of the phase 3, multicentre, double-blind, placebo-controlled, randomised PSUMMIT 2 trial. Annals of the rheumatic diseases. 2014;73(6):990-9.

21. Mease PJ, Gottlieb AB, van der Heijde D, FitzGerald O, Johnsen A, Nys M, et al. Efficacy and safety of abatacept, a T-cell modulator, in a randomised, double-blind, placebo-controlled, phase III study in psoriatic arthritis. Annals of the rheumatic diseases. 2017;76(9):1550-8.

22. Mease PJ, Genovese MC, Weinblatt ME, Peloso PM, Chen K, Othman AA, et al. Phase II Study of ABT-122, a Tumor Necrosis Factor- and Interleukin-17A-Targeted Dual Variable Domain Immunoglobulin, in Patients With Psoriatic Arthritis With an Inadequate Response to Methotrexate. Arthritis and Rheumatology. 2018;70(11):1778-89.

23. Mease PJ, Genovese MC, Greenwald MW, Ritchlin CT, Beaulieu AD, Deodhar A, et al. Brodalumab, an anti-IL17RA monoclonal antibody, in psoriatic arthritis. The New England journal of medicine. 2014;370(24):2295-306.

24. Mease P, Hall S, FitzGerald O, van der Heijde D, Merola JF, Avila-Zapata F, et al. Tofacitinib or Adalimumab versus Placebo for Psoriatic Arthritis. The New England journal of medicine.

2017;377(16):1537-50.

25. Gladman D, Rigby W, Azevedo VF, Behrens F, Blanco R, Kaszuba A, et al. Tofacitinib for Psoriatic Arthritis in Patients with an Inadequate Response to TNF Inhibitors. The New England journal of medicine. 2017;377(16):1525-36.

26. Antoni CE, Kavanaugh A, Kirkham B, Tutuncu Z, Burmester GR, Schneider U, et al. Sustained benefits of infliximab therapy for dermatologic and articular manifestations of psoriatic arthritis: results from the infliximab multinational psoriatic arthritis controlled trial (IMPACT). Arthritis and rheumatism. 2005;52(4):1227-36.

27. Antoni C, Krueger GG, de Vlam K, Birbara C, Beutler A, Guzzo C, et al. Infliximab improves signs and symptoms of psoriatic arthritis: results of the IMPACT 2 trial. Annals of the rheumatic diseases. 2005;64(8):1150-7.

28. Kavanaugh A, Antoni C, Krueger GG, Yan S, Bala M, Dooley LT, et al. Infliximab improves health related quality of life and physical function in patients with psoriatic arthritis. Annals of the rheumatic diseases. 2006;65(4):471-7.

29. Mease PJ, Ory P, Sharp JT, Ritchlin CT, Van den Bosch F, Wellborne F, et al. Adalimumab for long-term treatment of psoriatic arthritis: 2-year data from the Adalimumab Effectiveness in Psoriatic Arthritis Trial (ADEPT). Annals of the rheumatic diseases. 2009;68(5):702-9.

30. Genovese MC, Mease PJ, Thomson GT, Kivitz AJ, Perdok RJ, Weinberg MA, et al. Safety and efficacy of adalimumab in treatment of patients with psoriatic arthritis who had failed disease modifying antirheumatic drug therapy. The Journal of rheumatology. 2007;34(5):1040-50.

31. Mease PJ, Goffe BS, Metz J, VanderStoep A, Finck B, Burge DJ. Etanercept in the treatment of psoriatic arthritis and psoriasis: a randomised trial. Lancet (London, England). 2000;356(9227):38590.

32. Mease PJ, Woolley JM, Singh A, Tsuji W, Dunn M, Chiou CF. Patient-reported outcomes in a randomized trial of etanercept in psoriatic arthritis. The Journal of rheumatology. 2010;37(6):1221-7.

33. Gniadecki R, Robertson D, Molta CT, Freundlich B, Pedersen R, Li W, et al. Self-reported health outcomes in patients with psoriasis and psoriatic arthritis randomized to two etanercept regimens. Journal of the European Academy of Dermatology and Venereology : JEADV. 2012;26(11):1436-43.

34. Mease PJ, Gladman DD, Collier DH, Ritchlin CT, Helliwell PS, Liu L, et al. Etanercept and Methotrexate as Monotherapy or in Combination for Psoriatic Arthritis: Primary Results From a Randomized, Controlled Phase 3 Trial. Arthritis \& rheumatology (Hoboken, NJ). 2019.

35. Kavanaugh A, McInnes I, Mease P, Krueger GG, Gladman D, Gomez-Reino J, et al. Golimumab, a new human tumor necrosis factor alpha antibody, administered every four weeks as a 
subcutaneous injection in psoriatic arthritis: Twenty-four-week efficacy and safety results of a randomized, placebo-controlled study. Arthritis and rheumatism. 2009;60(4):976-86.

36. Kavanaugh A, Husni ME, Harrison DD, Kim L, Lo KH, Leu JH, et al. Safety and Efficacy of Intravenous Golimumab in Patients With Active Psoriatic Arthritis: Results Through Week TwentyFour of the GO-VIBRANT Study. Arthritis \& rheumatology (Hoboken, NJ). 2017;69(11):2151-61. 37. Gladman D, Fleischmann R, Coteur G, Woltering F, Mease PJ. Effect of certolizumab pegol on multiple facets of psoriatic arthritis as reported by patients: 24-week patient-reported outcome results of a phase III, multicenter study. Arthritis care \& research. 2014;66(7):1085-92.

38. McInnes IB, Sieper J, Braun J, Emery P, van der Heijde D, Isaacs JD, et al. Efficacy and safety of secukinumab, a fully human anti-interleukin-17A monoclonal antibody, in patients with moderateto-severe psoriatic arthritis: a 24-week, randomised, double-blind, placebo-controlled, phase II proof-of-concept trial. Annals of the rheumatic diseases. 2014;73(2):349-56.

39. Mease PJ, Mclnnes IB, Kirkham B, Kavanaugh A, Rahman P, van der Heijde D, et al. Secukinumab Inhibition of Interleukin-17A in Patients with Psoriatic Arthritis. The New England journal of medicine. 2015;373(14):1329-39.

40. Mclnnes IB, Mease PJ, Kirkham B, Kavanaugh A, Ritchlin CT, Rahman P, et al. Secukinumab, a human anti-interleukin-17A monoclonal antibody, in patients with psoriatic arthritis (FUTURE 2): a randomised, double-blind, placebo-controlled, phase 3 trial. Lancet (London, England). 2015;386(9999):1137-46.

41. Nash P, Mease PJ, Mclnnes IB, Rahman P, Ritchlin CT, Blanco R, et al. Efficacy and safety of secukinumab administration by autoinjector in patients with psoriatic arthritis: Results from a randomized, placebo-controlled trial (FUTURE 3). Arthritis Research and Therapy. 2018;20(1). 42. Mease PJ, van der Heijde D, Ritchlin CT, Okada M, Cuchacovich RS, Shuler CL, et al. Ixekizumab, an interleukin-17A specific monoclonal antibody, for the treatment of biologic-naive patients with active psoriatic arthritis: results from the 24-week randomised, double-blind, placebocontrolled and active (adalimumab)-controlled period of the phase III trial SPIRIT-P1. Annals of the rheumatic diseases. 2017;76(1):79-87.

43. Nash P, Kirkham B, Okada M, Rahman P, Combe B, Burmester GR, et al. Ixekizumab for the treatment of patients with active psoriatic arthritis and an inadequate response to tumour necrosis factor inhibitors: results from the 24-week randomised, double-blind, placebo-controlled period of the SPIRIT-P2 phase 3 trial. Lancet (London, England). 2017;389(10086):2317-27.

44. Gottlieb A, Menter A, Mendelsohn A, Shen YK, Li S, Guzzo C, et al. Ustekinumab, a human interleukin 12/23 monoclonal antibody, for psoriatic arthritis: randomised, double-blind, placebocontrolled, crossover trial. Lancet (London, England). 2009;373(9664):633-40.

45. McInnes IB, Kavanaugh A, Gottlieb AB, Puig L, Rahman P, Ritchlin C, et al. Efficacy and safety of ustekinumab in patients with active psoriatic arthritis: 1 year results of the phase 3 , multicentre, double-blind, placebo-controlled PSUMMIT 1 trial. Lancet (London, England). 2013;382(9894):780-9. 46. Araujo EG, Englbrecht M, Hoepken S, Finzel S, Kampylafka E, Kleyer A, et al. Effects of ustekinumab versus tumor necrosis factor inhibition on enthesitis: Results from the enthesial clearance in psoriatic arthritis (ECLIPSA) study. Seminars in arthritis and rheumatism. 2019;48(4):632-7.

47. Deodhar A, Gottlieb AB, Boehncke WH, Dong B, Wang Y, Zhuang Y, et al. Efficacy and safety of guselkumab in patients with active psoriatic arthritis: a randomised, double-blind, placebocontrolled, phase 2 study. Lancet (London, England). 2018;391(10136):2213-24.

48. Mease P, Genovese MC, Gladstein G, Kivitz AJ, Ritchlin C, Tak PP, et al. Abatacept in the treatment of patients with psoriatic arthritis: results of a six-month, multicenter, randomized, double-blind, placebo-controlled, phase II trial. Arthritis and rheumatism. 2011;63(4):939-48.

49. Mease PJ, Gladman DD, Keystone EC. Alefacept in combination with methotrexate for the treatment of psoriatic arthritis: results of a randomized, double-blind, placebo-controlled study. Arthritis and rheumatism. 2006;54(5):1638-45. 
50. Strand V, de Vlam K, Covarrubias-Cobos JA, Mease PJ, Gladman DD, Graham D, et al. Tofacitinib or adalimumab versus placebo: patient-reported outcomes from OPAL Broaden-a phase III study of active psoriatic arthritis in patients with an inadequate response to conventional synthetic disease-modifying antirheumatic drugs. RMD Open. 2019;5(1):e000806.

51. Strand V, de Vlam K, Covarrubias-Cobos JA, Mease PJ, Gladman DD, Chen L, et al. Effect of tofacitinib on patient-reported outcomes in patients with active psoriatic arthritis and an inadequate response to tumour necrosis factor inhibitors in the phase III, randomised controlled trial: OPAL Beyond. RMD Open. 2019;5(1):e000808.

52. Mease P, Coates LC, Helliwell PS, Stanislavchuk M, Rychlewska-Hanczewska A, Dudek A, et al. Efficacy and safety of filgotinib, a selective Janus kinase 1 inhibitor, in patients with active psoriatic arthritis (EQUATOR): results from a randomised, placebo-controlled, phase 2 trial. Lancet (London, England). 2018;392(10162):2367-77.

53. Mease PJ, Gottlieb AB, Berman A, Drescher E, Xing J, Wong R, et al. The Efficacy and Safety of Clazakizumab, an Anti-Interleukin-6 Monoclonal Antibody, in a Phase Ilb Study of Adults With Active Psoriatic Arthritis. Arthritis \& rheumatology (Hoboken, NJ). 2016;68(9):2163-73.

54. Mease PJ, Gladman DD, Ritchlin CT, Ruderman EM, Steinfeld SD, Choy EH, et al. Adalimumab for the treatment of patients with moderately to severely active psoriatic arthritis: results of a double-blind, randomized, placebo-controlled trial. Arthritis and rheumatism. 2005;52(10):3279-89. 
Table 1. Characteristics of included studies

\begin{tabular}{|c|c|c|c|c|c|c|c|c|c|c|c|}
\hline \multirow[t]{2}{*}{$\begin{array}{l}\text { Author / year/ } \\
\text { (study acronyms) }\end{array}$} & \multirow[t]{2}{*}{$\begin{array}{l}\text { Intervention/ } \\
\text { Comparator }\end{array}$} & \multirow[t]{2}{*}{$\begin{array}{l}\text { Sample size } \\
\text { (\% women) }\end{array}$} & \multirow{2}{*}{$\begin{array}{c}\text { PsA } \\
\text { duration } \\
\text { (years) }\end{array}$} & \multirow[t]{2}{*}{$\begin{array}{c}\text { TNFi } \\
\text { IR }\end{array}$} & \multirow[t]{2}{*}{ MTX use } & \multirow{2}{*}{$\begin{array}{c}\text { Primary } \\
\text { outcome/ } \\
\text { time point }\end{array}$} & \multirow{2}{*}{$\begin{array}{c}\text { Baseline } \\
\text { HAQ-DI } \\
\text { (mean) }\end{array}$} & \multicolumn{4}{|c|}{$\begin{array}{l}\text { Reported data for physical } \\
\text { function -PROM }\end{array}$} \\
\hline & & & & & & & & $\begin{array}{l}\text { HAQ- } \\
\text { DI }\end{array}$ & $\begin{array}{l}\text { HAQ } \\
-S\end{array}$ & $\begin{array}{l}\text { SF-36 } \\
\text { PCS }\end{array}$ & $\begin{array}{c}\text { SF-36 } \\
\text { PF }\end{array}$ \\
\hline \multicolumn{12}{|l|}{ TNF inhibitors } \\
\hline $\begin{array}{l}\text { Antoni, et al. } 2005 \\
\text { (IMPACT) (26) }\end{array}$ & $\begin{array}{l}\text { IFX } 5 \mathrm{mg} / \mathrm{kg} \\
\text { vs. PCB }\end{array}$ & $\begin{array}{l}\mathrm{N}=104 \\
(42.3)\end{array}$ & 11.4 & $0 \%$ & NA & $\begin{array}{l}\text { ACR20/ } \\
\text { Week 16 }\end{array}$ & 1.2 & Yes & No & No & No \\
\hline $\begin{array}{l}\text { Antoni, et al. 2005 } \\
\text { (IMPACT2) (27) }\end{array}$ & \multirow[t]{2}{*}{$\begin{array}{l}\text { IFX } 5 \mathrm{mg} / \mathrm{kg} \\
\text { vs. PCB }\end{array}$} & \multirow[t]{2}{*}{$\begin{array}{l}\mathrm{N}=200 \\
(39.0)\end{array}$} & \multirow[t]{2}{*}{8} & \multirow[t]{2}{*}{$0 \%$} & \multirow[t]{2}{*}{$46 \%$} & \multirow[t]{2}{*}{$\begin{array}{l}\text { ACR20/ } \\
\text { Week 14 }\end{array}$} & \multirow[t]{2}{*}{1.1} & Yes & No & - & No \\
\hline $\begin{array}{l}\text { Kavanaugh, et al. } \\
2006 \text { (IMPACT2) } \\
\text { (28) }\end{array}$ & & & & & & & & - & - & Yes & - \\
\hline $\begin{array}{l}\text { Mease, et al. } 2005 \\
\text { (ADEPT) (29) }\end{array}$ & $\begin{array}{l}\text { ADA 40mg Q2W } \\
\text { vs. PCB }\end{array}$ & $\begin{array}{l}\mathrm{N}=313 \\
(44.4)\end{array}$ & 9.5 & $0 \%$ & $51 \%$ & $\begin{array}{c}\text { ACR20/ } \\
\text { Week 12 } \\
\\
\Delta \text { in modified } \\
\text { Total Sharp } \\
\text { Score/ } \\
\text { Week 24 }\end{array}$ & 1.0 & Yes & No & Yes & No \\
\hline $\begin{array}{l}\text { Genovese, et al. } \\
2007(30)\end{array}$ & $\begin{array}{l}\text { ADA 40mg Q2W } \\
\text { vs. PCB }\end{array}$ & $\begin{array}{l}\mathrm{N}=100 \\
(46.0)\end{array}$ & 7.4 & $0 \%$ & $\begin{array}{c}47 \% \\
\text { (cs-DAMRD } \\
66 \% \text { ) }\end{array}$ & $\begin{array}{l}\text { ACR20/ } \\
\text { Week 12 }\end{array}$ & 1.0 & Yes & No & Yes & No \\
\hline $\begin{array}{l}\text { Mease, et al. } 2000 \\
(31)\end{array}$ & $\begin{array}{l}\text { ETN 25mg BIW } \\
\text { vs. PCB }\end{array}$ & $\begin{array}{l}\mathrm{N}=60 \\
(43.0)\end{array}$ & 9.3 & $0 \%$ & $47 \%$ & $\begin{array}{l}\text { PsARC/ } \\
\text { Week } 12\end{array}$ & 1.3 & Yes & No & No & No \\
\hline $\begin{array}{l}\text { Mease, et al. } 2010 \\
(32)\end{array}$ & $\begin{array}{l}\text { ETN 25mg BIW } \\
\text { vs. PCB }\end{array}$ & $\begin{array}{l}\mathrm{N}=205 \\
(49.0)\end{array}$ & 9.1 & $0 \%$ & $41.5 \%$ & $\begin{array}{l}\text { ACR20/ } \\
\text { Week 12 }\end{array}$ & 1.1 & Yes & No & Yes & No \\
\hline $\begin{array}{l}\text { Gniadecki, et al. } \\
2012 \\
\text { (PRESTA) (33) }\end{array}$ & $\begin{array}{l}\text { ETN 50mg } \\
\text { BIW/QW } \\
\text { vs. ETN 50mg } \\
\text { QW/QW }\end{array}$ & $\begin{array}{l}\mathrm{N}=752 \\
(37.0)\end{array}$ & 7.0 & NA & NA & $\begin{array}{c}\text { Psoriasis clear } \\
\text { or almost } \\
\text { clear/ } \\
\text { Week } 12\end{array}$ & 0.92 & Yes & No & No & No \\
\hline $\begin{array}{l}\text { Mease, et al. } 2019 \\
\text { (SEAM-PsA) (34) }\end{array}$ & $\begin{array}{l}\text { ETN 50mg QW } \\
\text { vs. ETN 50mg } \\
\text { QW plus MTX } \\
\text { vs. MTX alone }\end{array}$ & $\begin{array}{l}\mathrm{N}=851 \\
(50.8)\end{array}$ & 3.2 & $0 \%$ & $\begin{array}{c}100 \% \\
\text { (prior MTX use } \\
0 \% \text { ) }\end{array}$ & $\begin{array}{l}\text { ACR20/ } \\
\text { Week } 24\end{array}$ & 1.2 & Yes & No & Yes & No \\
\hline $\begin{array}{l}\text { Kavanaugh, et al. } \\
2009\end{array}$ & $\begin{array}{l}\text { GOL ( } 2 \text { doses }) \\
\text { vs. PCB }\end{array}$ & $\begin{array}{l}\mathrm{N}=405 \\
(39.8)\end{array}$ & 7.5 & $0 \%$ & $48 \%$ & $\begin{array}{l}\text { ACR20/ } \\
\text { Week 14 }\end{array}$ & 1.3 & Yes & No & Yes & No \\
\hline
\end{tabular}




\begin{tabular}{|c|c|c|c|c|c|c|c|c|c|c|c|}
\hline $\begin{array}{l}\text { (GO-REVEAL) } \\
\text { (35) }\end{array}$ & & & & & & & & & & & \\
\hline $\begin{array}{l}\text { Kavanaugh, et al. } \\
2017 \\
\text { (GO-VIBRANT) } \\
(36)\end{array}$ & $\begin{array}{l}\text { GOL IV 2mg/kg } \\
\text { vs. PCB }\end{array}$ & $\begin{array}{l}\mathrm{N}=480 \\
(48.0)\end{array}$ & 5.8 & $0 \%$ & $70 \%$ & $\begin{array}{l}\text { ACR20/ } \\
\text { Week 14 }\end{array}$ & 1.3 & Yes & No & Yes & No \\
\hline $\begin{array}{l}\text { Gladman, et al. } \\
2014 \\
\text { (RAPID-PsA) (37) }\end{array}$ & $\begin{array}{l}\text { CZP (2 doses) } \\
\text { vs. PCB }\end{array}$ & $\begin{array}{c}\mathrm{N}=409 \\
(55.3)\end{array}$ & 8.6 & $19.1 \%$ & $64.1 \%$ & $\begin{array}{c}\text { ACR20, } \\
\text { EULAR } \\
\text { response/ } \\
\text { Week 12 }\end{array}$ & 1.3 & Yes & No & Yes & No \\
\hline \multicolumn{12}{|l|}{ IL17 inhibitors } \\
\hline $\begin{array}{l}\text { McInnes, } 2014 \\
\text { (Phase II) (38) }\end{array}$ & $\begin{array}{l}\text { SEC }(10 \mathrm{mg} / \mathrm{kg}) \\
\text { vs. PCB }(\mathrm{N}=42)\end{array}$ & $\begin{array}{l}\mathrm{N}=42 \\
(64.0)\end{array}$ & NA & $35 \%$ & $\begin{array}{c}49 \% \\
\text { (any csDMARD } \\
51 \% \text { ) }\end{array}$ & $\begin{array}{l}\text { ACR20/ } \\
\text { Week } 6\end{array}$ & 1.5 & Yes & No & Yes & No \\
\hline $\begin{array}{l}\text { Mease, et al. } 2015 \\
\text { (FUTURE I) (39) }\end{array}$ & $\begin{array}{l}\text { SEC ( } 2 \text { doses }) \text { vs. } \\
\text { PCB }(\mathrm{N}=606)\end{array}$ & $\begin{array}{c}\mathrm{N}=606 \\
(54.5)\end{array}$ & NA & $29.4 \%$ & $60.7 \%$ & $\begin{array}{c}\text { ACR20/ } \\
\text { Week 24* }\end{array}$ & 1.2 & Yes & No & Yes & No \\
\hline $\begin{array}{l}\text { McInnes, et al. } \\
2015 \\
\text { (FUTURE II) (40) }\end{array}$ & $\begin{array}{l}\text { SEC (3 doses) } \\
\text { vs. PCB }\end{array}$ & \multirow[t]{2}{*}{$\begin{array}{l}\mathrm{N}=397 \\
(51.6)\end{array}$} & \multirow[t]{2}{*}{ NA } & \multirow[t]{2}{*}{$35 \%$} & \multirow[t]{2}{*}{$47 \%$} & \multirow[t]{2}{*}{$\begin{array}{c}\text { ACR20/ } \\
\text { Week 24* }\end{array}$} & \multirow[t]{2}{*}{1.2} & \multirow[t]{2}{*}{ Yes } & \multirow[t]{2}{*}{ No } & \multirow[t]{2}{*}{ Yes } & \multirow[t]{2}{*}{ No } \\
\hline $\begin{array}{l}\text { Kavanaugh, et al. } \\
2016 . \\
\text { (FUTURE II) (19) } \\
\text {-subgroup analysis }\end{array}$ & $\begin{array}{l}\text { TNFi-naïve } \\
\text { vs. TNFi-exposed }\end{array}$ & & & & & & & & & & \\
\hline $\begin{array}{l}\text { Nash, et al. } 2018 \\
\text { (FUTURE III) (41) }\end{array}$ & $\begin{array}{l}\text { SEC ( } 2 \text { doses }) \\
\text { vs. PCB }\end{array}$ & $\begin{array}{c}\mathrm{N}=414 \\
(54.8)\end{array}$ & 7.5 & $32 \%$ & $47.6 \%$ & $\begin{array}{l}\text { ACR20/ } \\
\text { Week 24 }\end{array}$ & 1.2 & Yes & No & Yes & No \\
\hline $\begin{array}{l}\text { Mease, et al. } 2017 \\
\text { (SPIRIT-P1) (42) }\end{array}$ & $\begin{array}{l}\text { IXE (2 doses }) \\
\text { vs. PCB } \\
\text { vs. ADA }\end{array}$ & $\begin{array}{l}\mathrm{N}=417 \\
(54.0)\end{array}$ & 6.7 & $0 \%$ & $\begin{array}{c}54.2 \% \\
\text { (any csDMARD } \\
64 \% \text { ) }\end{array}$ & $\begin{array}{c}\text { ACR20/ } \\
\text { Week 24* }\end{array}$ & 1.2 & Yes & No & Yes & No \\
\hline $\begin{array}{l}\text { Nash, et al. } 2017 \\
\text { (SPIRIT-P2) (43) }\end{array}$ & $\begin{array}{l}\text { IXE (2 doses) } \\
\text { vs. PCB }\end{array}$ & $\begin{array}{l}\mathrm{N}=363 \\
(53.4)\end{array}$ & 10.0 & $100 \%$ & $41 \%$ & $\begin{array}{c}\text { ACR20/ } \\
\text { Week 24* }\end{array}$ & 1.2 & Yes & No & Yes & No \\
\hline $\begin{array}{l}\text { Mease, et al. } 2014 \\
\text { Phase II (23) }\end{array}$ & $\begin{array}{l}\text { BRO ( } 2 \text { doses }) \\
\text { vs. PCB }\end{array}$ & $\begin{array}{l}\mathrm{N}=168 \\
(64.0)\end{array}$ & 8.7 & $51 \%$ & $50 \%$ & $\begin{array}{l}\text { ACR20/ } \\
\text { Week 12 }\end{array}$ & 1.3 & Yes & No & Yes & No \\
\hline \multicolumn{12}{|l|}{ IL12/23 inhibitors } \\
\hline $\begin{array}{l}\text { Gottlieb, et al. } 2009 \\
(44)\end{array}$ & $\begin{array}{l}\text { UST ( } 2 \text { doses }) \\
\text { vs. PCB }\end{array}$ & $\begin{array}{c}\mathrm{N}=146 \\
(43.8)\end{array}$ & 5.6 & $27.4 \%$ & $20.5 \%$ & $\begin{array}{l}\text { ACR20/ } \\
\text { Week 12 }\end{array}$ & 0.9 & Yes & No & No & No \\
\hline $\begin{array}{l}\text { McInnes, et al. } \\
2013 \\
\text { (PSUMMIT I) (45) }\end{array}$ & $\begin{array}{l}\text { UST ( } 2 \text { doses }) \\
\text { vs. PCB }\end{array}$ & $\begin{array}{l}N=615 \\
(46.3)\end{array}$ & 4.0 & $0 \%$ & $48 \%$ & $\begin{array}{l}\text { ACR20, } \\
\text { EULAR } \\
\text { response }\end{array}$ & 1.3 & Yes & No & Yes & No \\
\hline
\end{tabular}




\begin{tabular}{|c|c|c|c|c|c|c|c|c|c|c|c|}
\hline & & & & & & $\begin{array}{c}\text { PASI75/ } \\
\text { Week 24* }\end{array}$ & & & & & \\
\hline $\begin{array}{l}\text { Ritchlin, et al. } 2014 \\
\text { (PSUMMIT II) (20) }\end{array}$ & $\begin{array}{l}\text { UST } \\
(2 \text { doses }) \\
\text { vs. PCB }\end{array}$ & $\begin{array}{c}N=312 \\
(52.6)\end{array}$ & 5.1 & $57.7 \%$ & $49.7 \%$ & $\begin{array}{c}\text { ACR20, } \\
\text { EULAR } \\
\text { response } \\
\text { PASI75/ } \\
\text { Week 24* }\end{array}$ & 1.0 & Yes & No & Yes & No \\
\hline $\begin{array}{l}\text { Araugo, et al. } 2019 \\
\text { (ECLIPSA) (46) }\end{array}$ & $\begin{array}{l}\text { UST ( } 45 \mathrm{mg} \text { or } \\
100 \mathrm{mg} \text { if body } \\
\text { weight }>100 \mathrm{~kg} \text { ) } \\
\text { vs. TNFi }\end{array}$ & $\begin{array}{l}\mathrm{N}=47 \\
(40.4)\end{array}$ & 2.5 & $0 \%$ & $91.5 \%$ & $\begin{array}{c}\text { Enthesitis } \\
\text { resolution } \\
(\mathrm{SPARCC}=0) / \\
\text { Week } 24\end{array}$ & 1.0 & Yes & No & Yes & No \\
\hline \multicolumn{12}{|l|}{ IL23 inhibitors } \\
\hline $\begin{array}{l}\text { Deodhar, et al. } \\
2018(47)\end{array}$ & $\begin{array}{l}\text { GUS 100mg } \\
\text { vs. PCB }\end{array}$ & $\begin{array}{c}\mathrm{N}=149 \\
(49.0)\end{array}$ & 7.0 & $8.7 \%$ & $44.3 \%$ & $\begin{array}{c}\text { ACR20/ } \\
\text { Week24* }\end{array}$ & 1.4 & Yes & No & Yes & No \\
\hline \multicolumn{12}{|l|}{ T cell inhibition } \\
\hline $\begin{array}{l}\text { Mease, et al. } 2011 \\
\text { Phase II (48) }\end{array}$ & $\begin{array}{l}\text { ABT ( } 3 \text { doses }) \\
\text { vs. PCB }\end{array}$ & $\begin{array}{l}\mathrm{N}=170 \\
(44.2)\end{array}$ & 8.2 & $37.4 \%$ & $58.3 \%$ & $\begin{array}{l}\text { ACR20/ } \\
\text { Day } 169\end{array}$ & 1.2 & Yes & No & Yes & No \\
\hline $\begin{array}{l}\text { Mease, et al. } 2017 \\
\text { (ASTRAEA) (21) }\end{array}$ & $\begin{array}{l}\text { ABT } \\
\text { vs. PCB }\end{array}$ & $\begin{array}{c}\mathrm{N}=424 \\
(45.0)\end{array}$ & 8.5 & $61 \%$ & $60 \%$ & $\begin{array}{c}\text { ACR20/ } \\
\text { Week 24* }\end{array}$ & 1.3 & Yes & No & Yes & No \\
\hline $\begin{array}{l}\text { Mease, et al. } 2006 \\
(49)\end{array}$ & $\begin{array}{l}\text { ALC/MTX } \\
\text { vs. PCB/MTX }\end{array}$ & $\begin{array}{l}\mathrm{N}=185 \\
(61.1)\end{array}$ & 5 & $0 \%$ & $100 \%$ & $\begin{array}{l}\text { ACR20/ } \\
\text { Week 12 }\end{array}$ & 1.1 & Yes & No & No & No \\
\hline \multicolumn{12}{|l|}{ JAK inhibitors } \\
\hline $\begin{array}{l}\text { Mease, et al. } 2017 \\
\text { (OPAL Broaden) } \\
(24) \\
\text { Strand, et al. } 2019 \\
\text { (for SF-36 PCS) } \\
(50) \\
\end{array}$ & $\begin{array}{l}\text { TOF ( } 2 \text { doses }) \\
\text { vs. PCB } \\
\text { vs. ADA }\end{array}$ & $\begin{array}{c}\mathrm{N}=422 \\
(53.0)\end{array}$ & 6.1 & $0 \%$ & $83.9 \%$ & $\begin{array}{l}\text { ACR20/ } \\
3 \text { months }\end{array}$ & 1.1 & Yes & No & Yes & No \\
\hline $\begin{array}{l}\text { Gladman, et al. } \\
2017 \\
\text { (OPAL Beyond) } \\
\text { (25) } \\
\text { Strand, et al. } 2019 \\
\text { (for SF-36 PCS) } \\
\text { (51) }\end{array}$ & $\begin{array}{l}\text { TOF (2 doses) } \\
\text { vs. PCB }\end{array}$ & $\begin{array}{c}N=395 \\
(55.0)\end{array}$ & 9.4 & $100 \%$ & $73.6 \%$ & $\begin{array}{c}\text { ACR20, } \\
\Delta \text { in HAQ-DI/ } \\
3 \text { months }\end{array}$ & 1.3 & Yes & No & No & Yes \\
\hline $\begin{array}{l}\text { Mease, et al. } 2018 \\
\text { (EQUATOR) (52) } \\
\text { Phase II }\end{array}$ & $\begin{array}{l}\text { FIL 200mg } \\
\text { vs. PCB }\end{array}$ & $\begin{array}{c}N=131 \\
(50.4)\end{array}$ & 7.0 & $0 \%$ & $\begin{array}{c}54.2 \% \\
\text { (any csDMARD } \\
74 \% \text { ) } \\
\end{array}$ & $\begin{array}{l}\text { ACR20/ } \\
\text { Week } 16\end{array}$ & 1.4 & Yes & No & No & Yes \\
\hline
\end{tabular}




\begin{tabular}{|c|c|c|c|c|c|c|c|c|c|c|c|}
\hline \multicolumn{12}{|l|}{ IL6 inhibitors } \\
\hline $\begin{array}{l}\text { Mease, et al. } 2016 \\
\text { Phase II (53) }\end{array}$ & $\begin{array}{l}\text { CLZ ( } 3 \text { doses) } \\
\text { vs. PCB }\end{array}$ & $\begin{array}{l}\mathrm{N}=165 \\
(59.4)\end{array}$ & 7.1 & $0 \%$ & $69.1 \%$ & $\begin{array}{l}\text { ACR20/ } \\
\text { Week 16 }\end{array}$ & 1.4 & Yes & No & Yes & No \\
\hline \multicolumn{12}{|l|}{ Others } \\
\hline $\begin{array}{l}\text { Mease, et al. } 2018 \\
\text { Phase II (22) }\end{array}$ & $\begin{array}{l}\text { ABT-122 (2 } \\
\text { doses) } \\
\text { vs. PCB } \\
\text { vs. ADA }\end{array}$ & $\begin{array}{l}\mathrm{N}=240 \\
(49.6)\end{array}$ & 7.3 & $0 \%$ & $100 \%$ & $\begin{array}{l}\text { ACR20/ } \\
\text { Week } 12\end{array}$ & 1.3 & No & Yes & No & No \\
\hline
\end{tabular}

*early escape at Week 16;

Abbreviations: $\Delta$ : change; ACR: American College of Rheumatology Response criteria; ABT: abatacept; ADA: adalimumab; ALC: alefacept; BIW: twice a week; BRO: brodalumab; CI: confidence interval; CLZ: clazakizumab; csDMARD: conventional synthetic disease modifying anti-rheumatic drug; CZP: certolizumab pegol; ETN:

etanercept; EULAR: European League Against Rheumatism; FIL: filgotinib; GOL: golimumab; GUS: guselkumab; HAQ-DI: Health Assessment Questionnaire - Disability index; HAQ-S: Health Assessment Questionnaire - Spondyloarthritis; IFX: infliximab; IXE: ixekizumab; IV: intravenous; IL=interleukin; IR=inadequate response; MTX: methotrexate; NA: not available; NS: not significant; PASI: Psoriasis Area and Severity Index; PCB: placebo; PCS: physical component summary of SF-36; PF: physical

functioning domain of SF-36; PsA: psoriatic arthritis; PsARC: Psoriatic arthritis Response Criteria; QW: once a week; Q2W: once every 2 weeks; SD: standard deviation; SE: standard error; SEC: secukinumab; SF-36: Medical Outcomes Study 36-item Short Form Survey; SPARCC: Spondyloarthritis Research Consortium of Canada enthesitis index; TOF: tofacitinib; TNFi: tumor necrosis factor inhibitor; UST: ustekinumab; vs.: versus. 
Table 2. Effect size estimation for studies reporting HAQ-DI

\begin{tabular}{|c|c|c|c|c|}
\hline $\begin{array}{l}\text { Author / year/ } \\
\text { (study acronyms) }\end{array}$ & $\begin{array}{l}\text { Intervention/ comparator } \\
\text { (sample size, } \mathbf{N} \text { ) }\end{array}$ & $\begin{array}{l}\text { Primary outcome/ } \\
\text { time point }\end{array}$ & Effect sizes at primary endpoint (unless specified) & $\begin{array}{c}\text { a priori } \\
\text { hypothesis } \\
\text { satisfied } \\
\end{array}$ \\
\hline $\begin{array}{l}\text { Antoni, et al. 2005 } \\
\text { (IMPACT) (26) }\end{array}$ & $\begin{array}{l}\text { IFX 5mg/kg } \\
\text { vs. PCB } \\
(\mathrm{N}=104)\end{array}$ & $\begin{array}{l}\text { ACR20/ } \\
\text { Week } 16\end{array}$ & $\begin{array}{c}\text { SRM }^{\mathbb{I}} \text { (for improvement) }^{\delta} \text { at Week 16: } \\
\text { IFX }=6.07 \\
\text { PCB }=-0.19\end{array}$ & $1,2,3$ \\
\hline $\begin{array}{l}\text { Antoni, et al. 2005 } \\
\text { (IMPACT2) (27) }\end{array}$ & $\begin{array}{l}\text { IFX } 5 \mathrm{mg} / \mathrm{kg} \\
\text { vs. PCB } \\
(\mathrm{N}=200) \\
\end{array}$ & $\begin{array}{l}\text { ACR20/ } \\
\text { Week 14 }\end{array}$ & $\begin{array}{c}\text { SRM }^{\mathbb{I}} \text { (for improvement) }^{\delta} \text { at Week 14: } \\
\text { IFX }=1.08 \\
\text { PCB }=-0.19\end{array}$ & $1,2,3$ \\
\hline $\begin{array}{l}\text { Mease, et al. } 2005 \\
\text { (ADEPT) (54) }\end{array}$ & $\begin{array}{l}\text { ADA 40mg Q2W } \\
\text { vs. PCB } \\
(\mathrm{N}=313)\end{array}$ & $\begin{array}{c}\text { ACR20/ } \\
\text { Week 12; } \\
\Delta \text { in modified Total } \\
\text { Sharp Score/ } \\
\text { Week 24 } \\
\end{array}$ & $\begin{array}{c}\text { SRM at Week 12: } \\
\text { ADA }=-0.8 \\
\text { PCB }=0.2\end{array}$ & $1,2,3$ \\
\hline Genovese, et al. 2007 (30) & $\begin{array}{l}\text { ADA 40mg Q2W } \\
\text { vs. PCB } \\
(\mathrm{N}=100)\end{array}$ & $\begin{array}{l}\text { ACR20/ } \\
\text { Week } 12\end{array}$ & $\begin{array}{l}\text { SRM at Week 12: } \\
\text { ADA }=-0.6 \\
\text { PCB }=-0.33\end{array}$ & $1,2,3$ \\
\hline Mease, et al. 2000 (31) & $\begin{array}{l}\text { ETN 25mg BIW } \\
\text { vs. PCB } \\
(\mathrm{N}=60)\end{array}$ & $\begin{array}{l}\text { PsARC/ } \\
\text { Week } 12\end{array}$ & $\begin{array}{c}\mathrm{ES}_{2}{ }^{\ddagger} \text { at Week 12: } \\
\mathrm{ETN}=-0.547 \\
\mathrm{PCB}=-0.237\end{array}$ & $1,2,3$ \\
\hline Mease, et al. 2010 (32) & $\begin{array}{l}\text { ETN 25mg BIW } \\
\text { vs. PCB } \\
(\mathrm{N}=205)\end{array}$ & $\begin{array}{l}\text { ACR20/ } \\
\text { Week 12 }\end{array}$ & $\begin{array}{c}\text { Effect size for Week 12: NA } \\
\text { ES }_{2} \text { at Week } 24 \text { (end of double-blind phase): } \\
\text { ETN }=-0.597 \\
\text { PCB }=-0.098\end{array}$ & $1,2,3$ \\
\hline $\begin{array}{l}\text { Gniadecki, et al. } 2012 \\
\text { (PRESTA) (33) }\end{array}$ & $\begin{array}{l}\text { ETN 50mg BIW/QW } \\
\text { vs. ETN 50mg QW/QW } \\
(\mathrm{N}=752)\end{array}$ & $\begin{array}{l}\text { Psoriasis clear or } \\
\text { almost clear/ } \\
\text { Week } 12\end{array}$ & $\begin{array}{c}\text { ES}_{2} \text { at Week 12: } \\
\text { ETN 50mg BIW/QW: }-0.74 \\
\text { ETN 50mg QW/QW: }-0.69 \\
\end{array}$ & 1,3 \\
\hline $\begin{array}{l}\text { Mease, et al. } 2019 \\
\text { (SEAM-PsA) (34) }\end{array}$ & $\begin{array}{l}\text { ETN 50mg QW } \\
\text { vs. ETN 50mg QW plus MTX } \\
\text { vs. MTX alone } \\
(\mathrm{N}=851)\end{array}$ & $\begin{array}{l}\text { ACR20/ } \\
\text { Week 24 }\end{array}$ & $\begin{array}{c}\text { SRM at Week 24: } \\
\text { ETN }=-0.733 \\
\text { ETN plus MTX }=-0.685 \\
\text { MTX alone }=-0.646\end{array}$ & 1,3 \\
\hline $\begin{array}{l}\text { Kavanaugh, et al. } 2009 \\
\text { (GO-REVEAL) (35) }\end{array}$ & $\begin{array}{l}\text { GOL ( } 2 \text { doses }) \text { vs. PCB } \\
(\mathrm{N}=405)\end{array}$ & $\begin{array}{l}\text { ACR20/ } \\
\text { Week } 14\end{array}$ & $\begin{array}{c}\text { SRM at Week 14: } \\
\text { GOL } 100 \mathrm{mg}=-0.75 \\
\text { GOL } 50 \mathrm{mg}=-0.62 \\
\text { PCB }=-0.09\end{array}$ & $1,2,3$ \\
\hline Kavanaugh, et al. 2017 & GOL IV $2 \mathrm{mg} / \mathrm{kg}$ & ACR20/ & SRM at Week 14: & $1,2,3$ \\
\hline
\end{tabular}




\begin{tabular}{|c|c|c|c|c|}
\hline$(\mathrm{GO}-\mathrm{VIBRANT})(36)$ & $\begin{array}{l}\text { vs. PCB } \\
(\mathrm{N}=480)\end{array}$ & Week 14 & $\begin{array}{c}\text { GOL IV }=-1.13 \\
\text { PCB }=-0.26\end{array}$ & \\
\hline $\begin{array}{l}\text { Gladman, et al. } 2014 \\
\text { (RAPID-PsA) (37) }\end{array}$ & $\begin{array}{l}\text { CZP (2 doses }) \\
\text { vs. PCB }(\mathrm{N}=409)\end{array}$ & $\begin{array}{l}\text { ACR20, EULAR } \\
\text { response/ } \\
\text { Week } 12\end{array}$ & $\begin{array}{c}\text { SRM at Week 12: } \\
\text { CZP 400mg Q4W = -0.83 } \\
\text { CZP 200mg Q2W = -0.80 } \\
\text { PCB }=-0.44\end{array}$ & $1,2,3$ \\
\hline $\begin{array}{l}\text { McInnes, } 2014 \\
\text { (Phase II) (38) }\end{array}$ & $\begin{array}{l}\text { SEC }(10 \mathrm{mg} / \mathrm{kg}) \\
\text { vs. PCB }(\mathrm{N}=42)\end{array}$ & $\begin{array}{l}\text { ACR20/ } \\
\text { Week } 6\end{array}$ & $\begin{array}{c}\text { SRM at Week 6: } \\
\text { SEC }=-0.680 \\
\text { PCB }=0.018\end{array}$ & $1,2,3$ \\
\hline $\begin{array}{l}\text { Mease, et al. 2015 } \\
\text { (FUTURE I) (39) }\end{array}$ & $\begin{array}{l}\text { SEC ( } 2 \text { doses with loading) } \\
\text { vs. PCB }(\mathrm{N}=606)\end{array}$ & $\begin{array}{c}\text { ACR20/ } \\
\text { Week 24* }\end{array}$ & $\begin{array}{c}\text { SRM at Week 24: } \\
\text { SEC 150mg = -0.703 } \\
\text { SEC 75mg = -0.721 } \\
\text { PCB }=0.239\end{array}$ & $1,2,3$ \\
\hline $\begin{array}{l}\text { McInnes, et al. } 2015 \\
\text { FUTURE II) (40) }\end{array}$ & $\begin{array}{l}\text { SEC (3 doses) } \\
\text { vs. PCB }(\mathrm{N}=397)\end{array}$ & $\begin{array}{c}\text { ACR20/ } \\
\text { Week 24* }\end{array}$ & $\begin{array}{c}\text { SRM at Week 24: } \\
\text { SEC 300mg }=-1.12 \\
\text { SEC 150mg }=-0.96 \\
\text { SEC 75mg }=-0.644 \\
\text { PCB }=-0.522\end{array}$ & $1,2,3$ \\
\hline $\begin{array}{l}\text { Kavanaugh, et al. } 2016 \\
\text { (FUTURE II) (19) } \\
\text {-subgroup analysis }\end{array}$ & $\begin{array}{l}\text { TNFi-naïve } \\
\text { vs. TNFi-exposed }\end{array}$ & $\begin{array}{c}\text { ACR20/ } \\
\text { Week 24* }\end{array}$ & $\begin{array}{c}\text { SRM at Week } 24 \\
\text { (TNFi-naïve vs. -exposed): } \\
\text { SEC 300mg: }-1.20 \text { vs. }-1.02 \\
\text { SEC 150mg: }-1.15 \text { vs. }-0.71 \\
\text { SEC 75mg: }-0.77 \text { vs. }-0.44 \\
\text { PCB: }-0.63 \text { vs }-0.35\end{array}$ & 4 \\
\hline $\begin{array}{l}\text { Nash, et al. } 2018 \\
\text { (FUTURE III) (41) }\end{array}$ & $\begin{array}{l}\text { SEC (2 doses }) \\
\text { vs. PCB } \\
(\mathrm{N}=414)\end{array}$ & $\begin{array}{c}\text { ACR20/ } \\
\text { Week 24* }\end{array}$ & $\begin{array}{c}\text { SRM at Week 24: } \\
\text { SEC 300mg = -0.81 } \\
\text { SEC 150mg = -0.57 } \\
\text { PCB }=-0.24\end{array}$ & $1,2,3$ \\
\hline $\begin{array}{l}\text { Mease, et al. } 2017 \\
\text { (SPIRIT-P1) (42) }\end{array}$ & $\begin{array}{l}\text { IXE (2 doses) } \\
\text { vs. PCB } \\
\text { vs. ADA } \\
(\mathrm{N}=417)\end{array}$ & $\begin{array}{c}\text { ACR20/ } \\
\text { Week 24* }\end{array}$ & $\begin{array}{c}\text { SRM at Week 24: } \\
\text { IXE Q2W = } 0.98 \\
\text { IXE Q4W }=-0.85 \\
\text { PCB }=-0.35 \\
\text { ADA }=-0.74\end{array}$ & $1,2,3$ \\
\hline $\begin{array}{l}\text { Nash, et al. } 2017 \\
\text { (SPIRIT-P2) (43) }\end{array}$ & $\begin{array}{l}\text { IXE }(2 \text { doses }) \\
\text { vs. PCB } \\
(\mathrm{N}=363)\end{array}$ & $\begin{array}{c}\text { ACR20/ } \\
\text { Week 24* }\end{array}$ & $\begin{array}{c}\text { SRM at Week 24: } \\
\text { IXE Q2W = -0.36 } \\
\text { IXE Q4W = -0.55 } \\
\text { PCB }=-0.18\end{array}$ & $1,2,3$ \\
\hline
\end{tabular}




\begin{tabular}{|c|c|c|c|c|}
\hline $\begin{array}{l}\text { Mease, et al. } 2014 \\
\text { Phase II (23) }\end{array}$ & $\begin{array}{l}\text { BRO (2 doses) } \\
\text { vs. PCB }(\mathrm{N}=168)\end{array}$ & $\begin{array}{l}\text { ACR20/ } \\
\text { Week } 12\end{array}$ & $\begin{array}{c}\mathrm{SRM}^{\ddagger} \text { at Week 12: } \\
\text { BRO 280mg }=-0.60 \\
\text { BRO 140mg }=-0.38 \\
\quad P C B=-0.21\end{array}$ & $1,2,3$ \\
\hline Gottlieb, et al. 2009 (44) & $\begin{array}{l}\text { UST (2 doses) } \\
\text { vs. PCB }(\mathrm{N}=146)\end{array}$ & $\begin{array}{l}\text { ACR20/ } \\
\text { Week 12 }\end{array}$ & $\begin{array}{l}\text { SRM }^{\ddagger} \text { at Week 12: } \\
\text { UST }=-0.66 \\
\text { PCB }=-0.14 \\
\end{array}$ & $1,2,3$ \\
\hline $\begin{array}{l}\text { McInnes, et al. } 2013 \\
\text { (PSUMMIT I) (45) }\end{array}$ & $\begin{array}{l}\text { UST ( } 2 \text { doses }) \\
\text { vs. PCB } \\
(\mathrm{N}=615)\end{array}$ & $\begin{array}{c}\text { ACR20, } \\
\text { EULAR response } \\
\text { PASI75/ } \\
\text { Week 24* }\end{array}$ & $\begin{array}{l}\mathrm{SRM}^{\ddagger} \text { at Week 24: } \\
\text { UST 90mg }=-0.59 \\
\text { UST } 45 \mathrm{mg}=-0.62 \\
\mathrm{PCB}=-0.21\end{array}$ & $1,2,3$ \\
\hline $\begin{array}{l}\text { Ritchlin, et al. } 2014 \\
\text { (PSUMMIT II) (20) }\end{array}$ & $\begin{array}{l}\text { UST } \\
(2 \text { doses }) \\
\text { vs. PCB } \\
(\mathrm{N}=312)\end{array}$ & $\begin{array}{l}\text { ACR20, EULAR } \\
\text { response } \\
\text { PASI75/ } \\
\text { Week 24* }\end{array}$ & $\begin{array}{c}\text { SRM }^{¥} \text { at Week 24: } \\
\text { UST 90mg }=-0.66 \\
\text { UST } 45 \mathrm{mg}=-0.59 \\
\text { PCB }=0.00 \\
\text { SRM }{ }^{¥} \text { at Week } 24 \\
\text { Subgroups analysis (TNFi-naïve vs. -exposed): } \\
\text { UST 90mg }=-0.66 \text { vs. }-0.66 \\
\text { UST 45mg }=-0.66 \text { vs. }-0.59 \\
\text { PCB }=0 \text { vs. } 0\end{array}$ & $1,2,3$ \\
\hline $\begin{array}{l}\text { Araugo, et al. } 2019 \\
\text { (ECLIPSA) (46) }\end{array}$ & $\begin{array}{l}\text { UST }(45 \mathrm{mg} \text { or } 100 \mathrm{mg} \text { if body } \\
\text { weight }>100 \mathrm{~kg}) \\
\text { vs. TNFi }(\mathrm{N}=47)\end{array}$ & $\begin{array}{c}\text { Enthesitis resolution } \\
(\text { SPARCC }=0) \\
\text { at Week } 24\end{array}$ & $\begin{array}{c}\mathrm{ES}_{2}{ }^{\ddagger} \text { at Week 24: } \\
\mathrm{UST}=-1.81 \\
\mathrm{TNFi}=-1.74\end{array}$ & $1,2,3$ \\
\hline Deodhar, et al. 2018 (47) & $\begin{array}{l}\text { GUS 100mg } \\
\text { vs. PCB } \\
(\mathrm{N}=149)\end{array}$ & $\begin{array}{c}\text { ACR20/ } \\
\text { Week 24* }\end{array}$ & $\begin{array}{c}\text { SRM at Week 24: } \\
\text { GUS }=-0.82 \\
\text { PCB }=-0.11\end{array}$ & $1,2,3$ \\
\hline $\begin{array}{l}\text { Mease, et al. } 2011 \\
\text { Phase II (48) }\end{array}$ & $\begin{array}{l}\text { ABT (3 doses) } \\
\text { vs. PCB }(\mathrm{N}=170)\end{array}$ & Day 169 & Insufficient data for effect size calculation & none \\
\hline $\begin{array}{l}\text { Mease, et al. } 2017 \\
(\text { ASTRAEA) (21) }\end{array}$ & $\begin{array}{l}\text { ABT } \\
\text { vs. PCB }(\mathrm{N}=424)\end{array}$ & $\begin{array}{c}\text { ACR20/ } \\
\text { Week 24* }\end{array}$ & $\begin{array}{c}\mathrm{SRM}^{¥} \text { at Week } 24: \\
\mathrm{ABT}=-0.69 \\
\mathrm{PCB}=-0.40 \\
\\
\text { SRM }^{¥} \text { at Week } 24: \\
\text { Subgroup analysis: } \\
\text { (TNFi-naïve vs. -exposed) } \\
\mathrm{ABT}=-0.62 \text { vs. }-0.64 \\
\mathrm{PCB}=-0.39 \text { vs. }-0.34\end{array}$ & 1,3 \\
\hline Mease, et al. 2006 (49) & ALC/MTX & ACR20/ & $\% \Delta$ in HAQ-DI: & none \\
\hline
\end{tabular}




\begin{tabular}{|c|c|c|c|c|}
\hline & $\begin{array}{l}\text { vs. PCB/MTX } \\
(\mathrm{N}=185)\end{array}$ & Week 12 & $\begin{array}{c}\text { ALC/MTX }=-24.5 \% \\
\text { PCB/MTX }=-7.7 \% \\
(\mathrm{NS}) \\
\text { Inadequate data for ES calculations }\end{array}$ & \\
\hline $\begin{array}{l}\text { Mease, et al. } 2017 \\
\text { (OPAL Broaden) (24) }\end{array}$ & $\begin{array}{l}\text { TOF ( } 2 \text { doses }) \\
\text { vs. PCB } \\
\text { vs. ADA } \\
(\mathrm{N}=422)\end{array}$ & $\begin{array}{l}\text { ACR20/ } \\
3 \text { months }\end{array}$ & $\begin{array}{c}\text { SRM at } 3 \text { months: } \\
\text { TOF } 10 \mathrm{mg}=-0.78 \\
\text { TOF } 5 \mathrm{mg}=-0.69 \\
\text { PCB }=-0.36 \\
\text { ADA }=-0.76\end{array}$ & $1,2,3$ \\
\hline $\begin{array}{l}\text { Gladman, et al. } 2017 \\
\text { (OPAL Beyond) (25) }\end{array}$ & $\begin{array}{l}\text { TOF ( } 2 \text { doses }) \\
\text { vs. PCB } \\
(\mathrm{N}=395)\end{array}$ & $\begin{array}{c}\text { ACR20, } \\
\Delta \text { in HAQ-DI/ } \\
3 \text { months }\end{array}$ & $\begin{array}{l}\text { SRM at } 3 \text { months: } \\
\text { TOF } 10 \mathrm{mg}=-0.64 \\
\text { TOF }=-0.70 \\
\text { PCB }=-0.26\end{array}$ & $1,2,3$ \\
\hline $\begin{array}{l}\text { Mease, et al. } 2018 \\
\text { (EQUATOR) (52) } \\
\text { Phase II }\end{array}$ & $\begin{array}{l}\text { FIL 200mg } \\
\text { vs. PCB } \\
(\mathrm{N}=131)\end{array}$ & $\begin{array}{l}\text { ACR20/ } \\
\text { Week 16 }\end{array}$ & $\begin{array}{c}\text { SRM at Week 16: } \\
\text { FIL }=-1.14 \\
\text { PCB }=-0.56 \\
\text { ES }_{2} \text { at Week 16: } \\
\text { FIL }=-1.04 \\
\text { PCB }=-0.47\end{array}$ & $1,2,3$ \\
\hline $\begin{array}{l}\text { Mease, et al. } 2016 \\
\text { Phase II (53) }\end{array}$ & $\begin{array}{l}\text { CLZ (3 doses) } \\
\text { vs. PCB } \\
(\mathrm{N}=165)\end{array}$ & $\begin{array}{l}\text { ACR20/ } \\
\text { Week } 16\end{array}$ & $\begin{array}{c}\text { SRM }^{\ddagger} \text { at Week 16: } \\
\text { CLZ 200mg }=-0.51 \\
\text { CLZ 100mg }=-0.77 \\
\text { CLZ 50mg }=-0.83 \\
\text { PCB }=-0.52\end{array}$ & $1,2,3$ \\
\hline
\end{tabular}

${ }^{\text {II }}$ SRM calculated using percentage change score and SD of percentage change; ${ }^{\delta}$ SRM for improvement, a negative value indicate deterioration; ${ }^{\ddagger}$ Effect sizes estimated based on mean and SD of change scores calculated from median and IQR from original publication; * early escape for patients with inadequate response in the PCB group to active treatment group at Week 16; ** option to switch TNFi at Week 24.

Abbreviations: $\Delta$ : change; ACR: American College of Rheumatology Response criteria; ABT: abatacept; ADA: adalimumab; ALC: alefacept; BIW: twice a week; BRO: brodalumab; CI: confidence interval; CLZ: clazakizumab; CZP: certolizumab pegol; ES 2 : Effect size 2 (the mean difference divided by the pooled standard deviation, i.e Cohen's $d$ ); ETN: etanercept; EULAR: European League Against Rheumatism; FIL: filgotinib; GOL: golimumab; GUS: guselkumab; HAQ-DI: Health Assessment Questionnaire - Disability Index; IFX: infliximab; IXE: ixekizumab; IV: intravenous; IQR: interquartile range; MTX: methotrexate; NA: not available; NS: not significant; PASI: Psoriasis Area and Severity Index; PCB: placebo; PsARC: Psoriatic arthritis Response Criteria; QW: once a week; Q2W: once every 2-week; SD: standard deviation; SE: standard error; SEC: secukinumab; SPARCC: Spondyloarthritis Research Consortium of Canada enthesitis index; SRM: Standardized response mean (mean difference divided by the standard deviation of the differences between baseline and assessment end point); TOF: tofacitinib; TNFi: tumor necrosis factor inhibitor; UST: ustekinumab; vs.: versus. 
Table 3. Effect size estimation for studies reported HAQ-S

\begin{tabular}{|l|l|c|c|c|}
\hline $\begin{array}{l}\text { Author / year/ } \\
\text { (study acronyms) }\end{array}$ & $\begin{array}{l}\text { Intervention/ comparator } \\
\text { (sample size, N) }\end{array}$ & $\begin{array}{c}\text { Primary } \\
\text { outcome/ } \\
\text { time point }\end{array}$ & PROM & $\begin{array}{c}\text { E priori } \\
\text { hypothesis } \\
\text { satisfied }\end{array}$ \\
\hline Mease, et al. 2018 & ABT-122 (2 doses) & ACR20/ & HAQ-S & ES at $_{1}$ Week 12: \\
Phase II (22) & vs. PCB & Week 12 & ABT-122 240mg: -0.93 \\
& vs. ADA & & ABT-122 120mg: -0.92 \\
& (N=240) & & PCB: -0.47 \\
ADA: -0.97 & \\
\hline
\end{tabular}

Abbreviations: ACR: American College of Rheumatology Response criteria; ADA: adalimumab; ES 1 : Effect size 1 (the mean difference divided by standard deviation of baseline score); HAQ-S: Health Assessment Questionnaire - Spondyloarthritis; PCB: placebo; vs.: versus. 
Table 4. Effect size estimation for studies reporting SF-36 PCS

\begin{tabular}{|c|c|c|c|c|}
\hline $\begin{array}{l}\text { Author / year/ } \\
\text { (study acronyms) }\end{array}$ & $\begin{array}{l}\text { Intervention/ } \\
\text { comparator } \\
\text { (sample size, } \mathbf{N})\end{array}$ & $\begin{array}{l}\text { Primary Outcome/ } \\
\text { Time point }\end{array}$ & Effect sizes at primary end point (unless specified) & $\begin{array}{c}\text { Fulfilling of } a \\
\text { priori hypothesis }\end{array}$ \\
\hline $\begin{array}{l}\text { Antoni, et al. 2005 } \\
\text { (IMPACT2) (27) }\end{array}$ & $\begin{array}{l}\text { IFX 5mg/kg } \\
\text { vs. PCB } \\
(\mathrm{N}=200)\end{array}$ & $\begin{array}{l}\text { ACR20/ } \\
\text { Week 14 }\end{array}$ & $\begin{array}{c}\text { SRM at Week 14: } \\
\text { IFX }=0.98 \\
\text { PCB }=0.13\end{array}$ & $1,2,3$ \\
\hline $\begin{array}{l}\text { Mease, et al. } 2005 \\
(\mathrm{ADEPT})(54)\end{array}$ & $\begin{array}{l}\text { ADA 40mg Q2W } \\
\text { vs. PCB } \\
(\mathrm{N}=313)\end{array}$ & $\begin{array}{c}\text { ACR20/ } \\
\text { Week 12; } \\
\Delta \text { in modified Total Sharp } \\
\text { Score/ } \\
\text { Week 24 }\end{array}$ & $\begin{array}{c}\text { SRM at Week 12: } \\
\text { ADA }=0.93 \\
\text { PCB }=0.16\end{array}$ & $1,2,3$ \\
\hline Genovese, et al. 2007 (30) & $\begin{array}{l}\text { ADA 40mg Q2W } \\
\text { vs. PCB } \\
(\mathrm{N}=100)\end{array}$ & $\begin{array}{l}\text { ACR20/ } \\
\text { Week 12 }\end{array}$ & $\begin{array}{c}\text { SRM at Week 12: } \\
\text { ADA }=0.67 \\
\text { PCB }=0.39\end{array}$ & $1,2,3$ \\
\hline Mease, et al. 2010 (32) & $\begin{array}{l}\text { ETN 25mg BIW } \\
\text { vs. PCB } \\
(\mathrm{N}=205)\end{array}$ & $\begin{array}{l}\text { ACR20/ } \\
\text { Week 12 }\end{array}$ & $\begin{array}{c}\text { SRM at Week 12: NA } \\
\text { ES }_{2} \text { at Week 24 (end of double-blind phase): } \\
\text { ETN }=0.880 \\
\text { PCB }=0.073\end{array}$ & $1,2,3$ \\
\hline $\begin{array}{l}\text { Mease, et al. } 2019 \\
\text { (SEAM-PsA) (34) }\end{array}$ & $\begin{array}{l}\text { ETN 50mg QW } \\
\text { vs. ETN 50mg QW plus } \\
\text { MTX } \\
\text { vs. MTX alone } \\
(\mathrm{N}=851) \\
\end{array}$ & $\begin{array}{l}\text { ACR20/ } \\
\text { Week 24 }\end{array}$ & $\begin{array}{c}\text { SRM at Week 24: } \\
\text { ETN }=0.832 \\
\text { ETN plus MTX }=0.813 \\
\text { MTX alone }=0.629\end{array}$ & $1,2,3$ \\
\hline $\begin{array}{l}\text { Kavanaugh, et al. } 2009 \\
\text { (GO-REVEAL) (35) }\end{array}$ & $\begin{array}{l}\text { GOL ( } 2 \text { doses }) \\
\text { vs. } \mathrm{PCB} \\
(\mathrm{N}=405)\end{array}$ & $\begin{array}{l}\text { ACR20/ } \\
\text { Week 14 }\end{array}$ & $\begin{array}{c}\text { SRM at Week 14: } \\
\text { GOL 100mg = 0.82 } \\
\text { GOL 50mg }=0.74 \\
\text { PCB }=0.08\end{array}$ & $1,2,3$ \\
\hline $\begin{array}{l}\text { Kavanaugh, et al. } 2017 \\
\text { (GO-VIBRANT) (36) }\end{array}$ & $\begin{array}{l}\text { GOL IV } 2 \mathrm{mg} / \mathrm{kg} \\
\text { vs. PCB } \\
(\mathrm{N}=480)\end{array}$ & $\begin{array}{l}\text { ACR20/ } \\
\text { Week 14 }\end{array}$ & $\begin{array}{c}\text { SRM at Week 14: } \\
\text { GOL IV = 1.14 } \\
\text { PCB }=0.46\end{array}$ & $1,2,3$ \\
\hline $\begin{array}{l}\text { Gladman, et al. } 2014 \\
\text { (RAPID-PsA) (37) }\end{array}$ & $\begin{array}{l}\text { CZP (2 doses }) \\
\text { vs. PCB } \\
(\mathrm{N}=409)\end{array}$ & $\begin{array}{l}\text { ACR20, EULAR response/ } \\
\text { Week } 12\end{array}$ & $\begin{array}{c}\text { SRM at Week 12: } \\
\text { CZP 400mg Q4W =0.87 } \\
\text { CZP 200mg Q2W = } 0.82 \\
\text { PCB }=0.30\end{array}$ & $1,2,3$ \\
\hline
\end{tabular}




\begin{tabular}{|c|c|c|c|c|}
\hline $\begin{array}{l}\text { McInnes IB, } 2014 \\
\text { (Phase II) (38) }\end{array}$ & $\begin{array}{l}\text { SEC }(10 \mathrm{mg} / \mathrm{kg}) \\
\text { vs. PCB } \\
(\mathrm{N}=42) \\
\end{array}$ & $\begin{array}{l}\text { ACR20/ } \\
\text { Week } 6\end{array}$ & $\begin{array}{c}\text { SRM at Week 6: } \\
\text { SEC }=0.541 \\
\text { PCB }=-0.017\end{array}$ & $1,2,3$ \\
\hline $\begin{array}{l}\text { Mease, et al. } 2015 \\
\text { (FUTURE I) (39) }\end{array}$ & $\begin{array}{l}\text { SEC }(2 \text { doses with } \\
\text { loading }) \\
\text { vs. PCB } \\
(\mathrm{N}=606)\end{array}$ & $\begin{array}{c}\text { ACR20/ } \\
\text { Week 24* }\end{array}$ & $\begin{array}{c}\text { SRM at Week 24: } \\
\text { SEC } 150 \mathrm{mg}=0.785 \\
\text { SEC } 75 \mathrm{mg}=0.732 \\
\text { PCB }=0.178\end{array}$ & $1,2,3$ \\
\hline $\begin{array}{l}\text { McInnes, et al. } 2015 \\
\text { FUTURE II) (40) }\end{array}$ & $\begin{array}{l}\text { SEC (3 doses) } \\
\text { vs. PCB } \\
(\mathrm{N}=397)\end{array}$ & $\begin{array}{c}\text { ACR20/ } \\
\text { Week 24* }\end{array}$ & $\begin{array}{c}\text { SRM at Week 24: } \\
\text { SEC 300mg }=0.98 \\
\text { SEC } 150 \mathrm{mg}=0.875 \\
\text { SEC } 75 \mathrm{mg}=0.587 \\
\text { PCB }=0.203\end{array}$ & $1,2,3$ \\
\hline $\begin{array}{l}\text { Kavanaugh, et al. } 2016 . \\
\text { (FUTURE II) (19) } \\
\text {-subgroup analysis }\end{array}$ & $\begin{array}{l}\text { TNFi-naïve } \\
\text { vs. TNFi-exposed }\end{array}$ & $\begin{array}{c}\text { ACR20/ } \\
\text { Week 24* }\end{array}$ & $\begin{array}{c}\text { SRM at Week } 24: \\
\text { (TNFi-naïve vs. -exposed): } \\
\text { SEC 300mg: } 1.07 \text { vs. } 0.95 \\
\text { SEC 150mg: } 1.07 \text { vs. } 0.60 \\
\text { SEC 75mg: } 0.71 \text { vs. } 0.45 \\
\text { PCB: } 0.22 \text { vs } 0.27\end{array}$ & 4 \\
\hline $\begin{array}{l}\text { Nash, et al. } 2018 \\
\text { (FUTURE III) (41) }\end{array}$ & $\begin{array}{l}\text { SEC (2 doses }) \\
\text { vs. PCB } \\
(\mathrm{N}=414)\end{array}$ & $\begin{array}{c}\text { ACR20/ } \\
\text { Week 24* }\end{array}$ & $\begin{array}{c}\text { SRM at Week 24: } \\
\text { SEC 300mg }=0.93 \\
\text { SEC } 150 \mathrm{mg}=0.49 \\
\text { PCB }=0.30\end{array}$ & $1,2,3$ \\
\hline $\begin{array}{l}\text { Mease, et al. } 2017 \\
\text { (SPIRIT-P1) (42) }\end{array}$ & $\begin{array}{l}\text { IXE }(2 \text { doses }) \\
\text { vs. PCB } \\
\text { vs. ADA } \\
(\mathrm{N}=417)\end{array}$ & $\begin{array}{c}\text { ACR20/ } \\
\text { Week 24* }\end{array}$ & $\begin{array}{c}\text { SRM at Week 24: } \\
\text { IXE Q2W }=1.79 \\
\text { IXE Q4W = } 1.33 \\
\text { PCB }=0.55 \\
\text { ADA }=1.78\end{array}$ & $1,2,3$ \\
\hline $\begin{array}{l}\text { Nash, et al. } 2017 \\
\text { (SPIRIT-P2) (43) }\end{array}$ & $\begin{array}{l}\text { IXE ( } 2 \text { doses }) \\
\text { vs. PCB } \\
(\mathrm{N}=363)\end{array}$ & $\begin{array}{c}\text { ACR20/ } \\
\text { Week 24* }\end{array}$ & $\begin{array}{l}\text { SRM at Week 24: } \\
\text { IXE Q2W =0.30 } \\
\text { IXE Q4W =0.27 } \\
\quad \text { PCB }=0.06\end{array}$ & $1,2,3$ \\
\hline $\begin{array}{l}\text { Mease, et al. } 2014 \\
\text { Phase II (23) }\end{array}$ & $\begin{array}{l}\text { BRO }(2 \text { doses }) \\
\text { vs. PCB } \\
(\mathrm{N}=168)\end{array}$ & $\begin{array}{l}\text { ACR20/ } \\
\text { Week 12 }\end{array}$ & $\begin{array}{c}\text { Difference from PCB (95\% CI): } \\
\text { BRO 280mg: } 2.4(0.1 \text { to } 4.6) \\
\text { BRO 140mg: } 1.4(-0.8 \text { to } 3.6) \\
\text { Insufficient data for effect size calculation }\end{array}$ & none \\
\hline $\begin{array}{l}\text { McInnes, et al. } \\
2013 \\
\text { (PSUMMIT I) (45) }\end{array}$ & $\begin{array}{l}\text { UST ( } 2 \text { doses }) \\
\text { vs. PCB } \\
(\mathrm{N}=615)\end{array}$ & $\begin{array}{l}\text { ACR20, EULAR response, } \\
\text { PASI75/ } \\
\text { Week } 24^{*} \\
\end{array}$ & $\begin{array}{c}\text { SRM }^{¥} \text { at Week 24: } \\
\text { UST } 90 \mathrm{mg}=0.75 \\
\text { UST } 45 \mathrm{mg}=0.48 \\
\end{array}$ & $1,2,3$ \\
\hline
\end{tabular}




\begin{tabular}{|c|c|c|c|c|}
\hline & & & $\mathrm{PCB}=0.24$ & \\
\hline $\begin{array}{l}\text { Ritchlin, et al. } 2014 \\
\text { (PSUMMIT II) (20) }\end{array}$ & $\begin{array}{l}\text { UST ( } 2 \text { doses }) \\
\text { vs. PCB } \\
(\mathrm{N}=312)\end{array}$ & $\begin{array}{c}\text { ACR20, EULAR response, } \\
\text { PASI75/ } \\
\text { Week 24* }\end{array}$ & $\begin{array}{c}\text { SRM }^{\ddagger} \text { at Week 24: } \\
\text { UST 90mg }=0.60 \\
\text { UST } 45 \mathrm{mg}=0.50 \\
\text { PCB }=0.30\end{array}$ & $1,2,3$ \\
\hline $\begin{array}{l}\text { Araugo, et al. } 2019 \\
\text { (ECLIPSA) (46) }\end{array}$ & $\begin{array}{l}\text { UST }(45 \mathrm{mg} \text { or } 100 \mathrm{mg} \text { if } \\
\text { body weight }>100 \mathrm{~kg}) \\
\text { vs. TNFi } \\
(\mathrm{N}=47)\end{array}$ & $\begin{array}{l}\text { Enthesitis resolution } \\
(\text { SPARCC }=0) / \\
\text { Week } 24\end{array}$ & $\begin{array}{c}\mathrm{ES}_{2}{ }^{\ddagger} \text { at Week 24: } \\
\mathrm{UST}=2.95 \\
\mathrm{TNFi}=1.56\end{array}$ & 1,3 \\
\hline Deodhar, et al. 2018 (47) & $\begin{array}{l}\text { GUS 100mg } \\
\text { vs. PCB } \\
(\mathrm{N}=149) \\
\end{array}$ & $\begin{array}{c}\text { ACR20/ } \\
\text { Week 24* }\end{array}$ & $\begin{array}{c}\text { SRM at Week 24: } \\
\text { GUS }=0.88 \\
\text { PCB }=0.06\end{array}$ & $1,2,3$ \\
\hline $\begin{array}{l}\text { Mease, et al. } 2011 \\
\text { Phase II (48) }\end{array}$ & $\begin{array}{l}\text { ABT ( } 3 \text { doses }) \\
\text { vs. PCB } \\
(\mathrm{N}=170)\end{array}$ & $\begin{array}{l}\text { ACR20/ } \\
\text { Day } 169\end{array}$ & $\begin{array}{c}\text { SRM at Day 169: } \\
\text { ABT 30/10 mg/kg: } 0.59 \\
\text { ABT 10mg/kg: } 0.77 \\
\text { ABT } 3 \mathrm{mg} / \mathrm{kg}: 0.53 \\
\text { PCB: } 0.02\end{array}$ & $1,2,3$ \\
\hline $\begin{array}{l}\text { Mease, et al. } 2017 \\
\text { (ASTRAEA) (21) }\end{array}$ & $\begin{array}{l}\text { ABT } \\
\text { vs. PCB } \\
(\mathrm{N}=424) \\
\end{array}$ & $\begin{array}{c}\text { ACR20/ } \\
\text { Week 24* }\end{array}$ & $\begin{array}{l}\text { SRM }^{\ddagger} \text { at Week } 24: \\
\text { ABT: } 0.72 \\
\text { PCB: } 0.53 \\
\end{array}$ & $1,2,3$ \\
\hline $\begin{array}{l}\text { Strand, et al. } \\
2019 \text { (OPAL Broaden) } \\
(50)\end{array}$ & $\begin{array}{l}\text { TOF ( } 2 \text { doses }) \\
\text { vs. PCB } \\
\text { vs. ADA } \\
(\mathrm{N}=422)\end{array}$ & $\begin{array}{l}\text { ACR20/ } \\
3 \text { months }\end{array}$ & $\begin{array}{c}\text { SRM at } 3 \text { months: } \\
\text { TOF } 10 \mathrm{mg}=0.75 \\
\text { TOF } 5 \mathrm{mg}=0.73 \\
\text { PCB }=0.33 \\
\text { ADA }=0.81\end{array}$ & $1,2,3$ \\
\hline $\begin{array}{l}\text { Strand, et al. } \\
2019 \text { (OPAL Beyond) (51) }\end{array}$ & $\begin{array}{l}\text { TOF ( } 2 \text { doses }) \\
\text { vs. PCB } \\
(\mathrm{N}=395)\end{array}$ & $\begin{array}{c}\text { ACR20, } \\
\Delta \text { in HAQ-DI/ } \\
3 \text { months }\end{array}$ & $\begin{array}{l}\text { SRM at } 3 \text { month: } \\
\text { TOF } 10 \mathrm{mg}=0.67 \\
\text { TOF } 5 \mathrm{mg}=0.67 \\
\text { PCB }=0.22\end{array}$ & $1,2,3$ \\
\hline $\begin{array}{l}\text { Mease, et al. } 2016 \\
\text { Phase II (53) }\end{array}$ & $\begin{array}{l}\text { CLZ (3 doses) } \\
\text { vs. PCB } \\
(\mathrm{N}=165)\end{array}$ & $\begin{array}{l}\text { ACR20/ } \\
\text { Week 16 }\end{array}$ & $\begin{array}{c}\text { SRM }^{¥} \text { at Week 16: } \\
\text { CLZ 200mg }=0.52 \\
\text { CLZ 100mg }=0.59 \\
\text { CLZ 50mg }=0.82 \\
\text { PCB }=0.57\end{array}$ & $1,2,3$ \\
\hline
\end{tabular}

${ }^{\pi}$ SRM calculated using percentage change score and SD of percentage change; ${ }^{\delta}$ SRM for improvement, a negative value indicate deterioration; ${ }^{¥}$ Effect sizes estimated based on mean and SD of change scores calculated from median and IQR from original publication; * early escape for patients with inadequate response in the PCB group to active treatment group at Week 16;** option to switch TNFi at Week 24. 
Abbreviations: $\Delta$ : change. ACR: American College of Rheumatology Response criteria; ABT: abatacept; ADA: adalimumab; ALC: alefacept; BIW: twice a week; BRO: brodalumab; CI: confidence interval; CLZ: clazakizumab; CZP: certolizumab pegol; ES 2 : Effect size 2 (the mean difference divided by the pooled standard deviation, i.e Cohen's $d$ ); ETN: etanercept; EULAR: European League Against Rheumatic Diseases; GOL: golimumab; GUS: guselkumab; IFX: infliximab; IXE: ixekizumab; IV:

intravenous; IQR: interquartile range; MTX: methotrexate; NA: not available; PASI: psoriasis area and severity index; PCB: placebo; PCS: physical component summary of

SF-36; PF: physical functioning domain of SF-36; QW: once a week; Q2W: once every 2-week; SD: standard deviation; SE: standard error; SEC: secukinumab; SF-36:

Medical Outcome Survey Short Form 36 items; SPARCC: Spondyloarthritis Research Consortium of Canada enthesitis index; SRM: Standardized response mean (mean difference divided by the standard deviation of the differences between baseline and assessment end point); TNFi: tumor necrosis factor inhibitor; UST: ustekinumab; vs.: versus. 
Table 5. Effect size estimation for studies reporting SF-36 PF.

\begin{tabular}{|c|c|c|c|c|}
\hline $\begin{array}{l}\text { Author / year/ } \\
\text { (study acronyms) }\end{array}$ & $\begin{array}{l}\text { Intervention/ } \\
\text { comparator } \\
(\text { sample size, } \mathbf{N})\end{array}$ & $\begin{array}{c}\text { Primary } \\
\text { outcome/ } \\
\text { time point }\end{array}$ & $\begin{array}{c}\text { Effect sizes at primary } \\
\text { endpoint (unless specified) }\end{array}$ & $\begin{array}{c}\text { Fulfilling } \\
\text { of } a \text { priori } \\
\text { hypothesis }\end{array}$ \\
\hline $\begin{array}{l}\text { Antoni, et al. } 2007 \\
\text { (IMPACT2) (27) }\end{array}$ & $\begin{array}{l}\text { IFX } 5 \mathrm{mg} / \mathrm{kg} \\
\text { vs. PCB } \\
(\mathrm{N}=200)\end{array}$ & $\begin{array}{l}\text { ACR20/ } \\
\text { Week 14 }\end{array}$ & $\begin{array}{l}\text { Insufficient data for effect } \\
\text { size calculation }\end{array}$ & 1,2 \\
\hline $\begin{array}{l}\text { Mease, et al. } 2017 \\
\text { (OPAL Broaden) }\end{array}$ & $\begin{array}{l}\text { TOF ( } 2 \text { doses }) \\
\text { vs. PCB } \\
\text { vs. ADA } \\
(\mathrm{N}=422)\end{array}$ & $\begin{array}{l}\text { ACR20/ } \\
3 \text { months }\end{array}$ & $\begin{array}{c}\text { SRM at } 3 \text { months: } \\
\text { TOF } 10 \mathrm{mg}=0.64 \\
\text { TOF } 5 \mathrm{mg}=0.64 \\
\text { PCB }=0.23 \\
\text { ADA }=0.58\end{array}$ & 1,3 \\
\hline $\begin{array}{l}\text { Gladman, et al. } \\
2017 \\
\text { (OPAL Beyond) }\end{array}$ & $\begin{array}{l}\text { TOF ( } 2 \text { doses }) \\
\text { vs. } \mathrm{PCB} \\
(\mathrm{N}=395)\end{array}$ & $\begin{array}{c}\text { ACR20, } \\
\Delta \text { in HAQ-DI/ } \\
3 \text { months }\end{array}$ & $\begin{array}{c}\text { SRM at } 3 \text { months: } \\
\text { TOF } 10 \mathrm{mg}=0.53 \\
\text { TOF } 5 \mathrm{mg}=0.64 \\
\text { PCB }=0.22\end{array}$ & 1,3 \\
\hline $\begin{array}{l}\text { Mease, et al. } 2018 \\
\text { (EQUATOR) } \\
\text { Phase II }\end{array}$ & $\begin{array}{l}\text { FIL 200mg } \\
\text { vs. PCB } \\
(\mathrm{N}=131)\end{array}$ & $\begin{array}{l}\text { ACR20/ } \\
\text { Week } 16\end{array}$ & $\begin{array}{l}\text { Insufficient data for effect } \\
\text { size calculation }\end{array}$ & 1,2 \\
\hline
\end{tabular}

II SRM calculated using percentage change score and SD of percentage change; ${ }^{\delta}$ SRM for improvement, a negative value indicate deterioration; ${ }^{¥}$ Effect sizes estimated based on mean and SD of change scores calculated from median and IQR from original publication; * early escape for patients with inadequate response in the control group to active treatment group at week 16; ** option to switch TNFi at Week 24.

Abbreviations: $\Delta$ : change; ACR: American College of Rheumatology Response criteria; ADA: adalimumab; $\mathrm{ES}_{2}$ : Effect size 2 (the mean difference divided by the pooled standard deviation, i.e Cohen's $d$ ); FIL: filgotinib; HAQ-DI: Health Assessment Questionnaire - Disability Index; IFX: infliximab; IQR: interquartile range; PCB: placebo; PF: physical functioning domain of SF-36; SD: standard deviation; SF-36: Medical Outcomes Study 36-item Short Form Survey; SRM: Standardized response mean (mean difference divided by the standard deviation of the differences between baseline and assessment end point); TOF: tofacitinib; vs.: versus. 
Table 6. Summary of Measurement Properties Table for clinical trial discrimination

\begin{tabular}{|c|c|c|c|c|}
\hline $1^{\text {st }}$ Author / year/ (study acronyms/ drug) & $\begin{array}{l}\text { HAQ- } \\
\text { DI }\end{array}$ & HAQ-S & $\begin{array}{l}\text { SF-36 } \\
\text { PCS }\end{array}$ & $\begin{array}{l}\text { SF-36 } \\
\text { PF }\end{array}$ \\
\hline Antoni, 2005 (IMPACT) & + & & & \\
\hline Antoni, 2005 (IMPACT2) & + & & + & \\
\hline Kavanaugh, 2006 (IMPACT2) & & & & + \\
\hline Mease, 2005 (ADEPT) & + & & + & \\
\hline Genovese, 2007 (ADA) & + & & + & \\
\hline Mease, $2000(\mathrm{ETN})$ & + & & + & \\
\hline Mease. $2010(\mathrm{ETN})$ & + & & + & \\
\hline Gniadecki, 2012 (PRESTA) & + & & 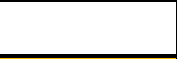 & \\
\hline Mease. 2019 (SEAM-PsA) & $+/-$ & & + & \\
\hline Kavanaugh, 2009 (GO-REVEAL) & + & & + & \\
\hline Kavanaugh, 2017 (GO-VIBRANT) & + & & + & \\
\hline Gladman, 2014 (RAPID-PsA) & + & & + & \\
\hline McInnes, 2014 (SEC) & + & & + & \\
\hline Mease, 2015 (FUTURE I) & + & & + & \\
\hline McInnes, 2015 FUTURE II) & + & & + & \\
\hline $\begin{array}{l}\text { Kavanaugh, } 2016 \text { (FUTURE II) } \\
\text {-subgroup analysis }\end{array}$ & + & & + & \\
\hline Nash P, 2018 (FUTURE III) & + & & + & \\
\hline Mease, 2017 (SPIRIT-P1) & + & & + & \\
\hline Nash, 2017 (SPIRIT-P2) & + & & + & \\
\hline Mease, 2014 (BRO) & + & & + & \\
\hline Gottlieb, 2009 (UST) & + & & 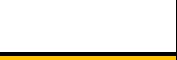 & \\
\hline McInnes, 2013 (PSUMMIT I) & + & & + & \\
\hline Ritchlin, 2014 (PSUMMIT II) & + & & + & \\
\hline Araugo, 2019 (ECLIPSA) & + & & + & \\
\hline Deodhar, 2018 (GUS) & + & & + & \\
\hline Mease, $2011(\mathrm{ABT})$ & $+/-$ & & + & \\
\hline Mease, 2017 (ASTRAEA) & + & & + & \\
\hline Mease, 2006 (ALC) & $+/-$ & & & \\
\hline Mease, 2017 (OPAL Broaden) & + & & & + \\
\hline Strand, 2019 (OPAL Broaden) & & & + & \\
\hline Gladman, 2017 (OPAL Beyond) & + & & & + \\
\hline Strand, 2019 (OPAL Beyond) & & & + & \\
\hline Mease, 2018 (EQUATOR) & + & & & + \\
\hline Mease, 2016 (CLZ) & + & & + & \\
\hline Mease, 2018 (ABT-122) & & + & & \\
\hline Total available articles & 31 & 1 & 26 & 4 \\
\hline Total articles for evidence synthesis & 29 & 1 & 25 & 2 \\
\hline Final rating & GREEN & AMBER & GREEN & AMBER \\
\hline
\end{tabular}

Color code in each box indicate study quality assessed by OMERACT good methods. GREEN means "yes, likely low risk of bias"; AMBER means "some cautions but can be used as evidence" and RED means "No, 
don't use as evidence". WHITE (empty boxes), indicates absence of information on that property from that study. (+) indicates findings of the study had adequate performance of the instrument; (+/-) indicates equivocal performance; (-) indicates poor performance (less than adequate). 


\section{Appendices}

Table A.1. Detailed search terms of the systematic review

Pubmed:

"Arthritis, Psoriatic"[Mesh] AND "randomized controlled trial"[publication type] AND

English[lang]

Scopus:

( TITLE-ABS-KEY ( psoriatic AND arthritis ) AND TITLE-ABS-

KEY ( randomized AND controlled AND trial) ) AND DOCTYPE (ar ) AND (LIMI

T-TO ( PUBSTAGE, "final" ) ) AND ( LIMIT-TO ( LANGUAGE, "English" ) ) 
Table A.2. Effect sizes estimation of all studies.

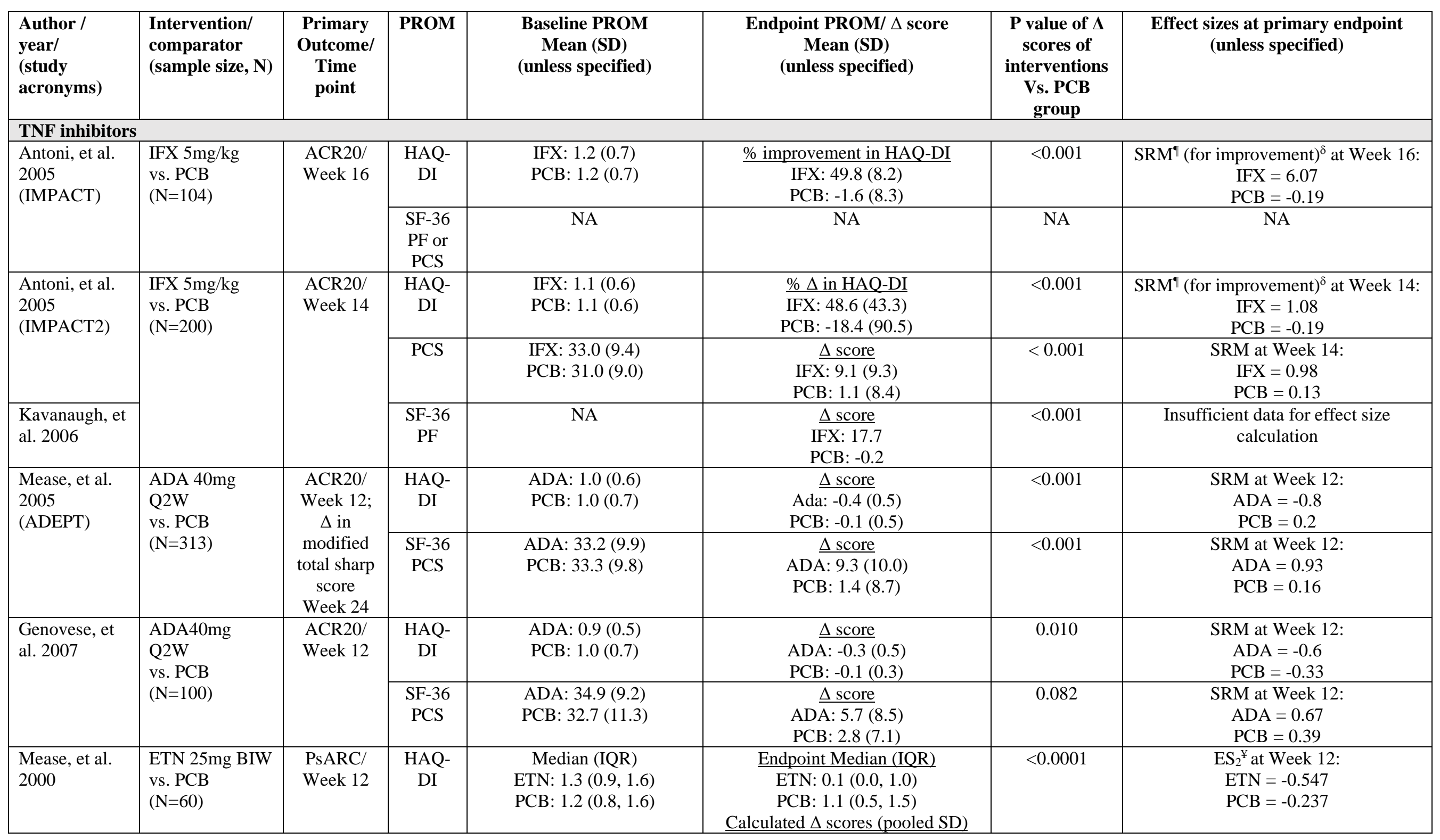




\begin{tabular}{|c|c|c|c|c|c|c|c|}
\hline & & & & & $\begin{array}{l}\text { ETN: }-0.367(0.671) \\
\text { PCB: }-0.167(0.705)\end{array}$ & & \\
\hline & & & $\begin{array}{l}\text { SF-36 } \\
\text { PF or } \\
\text { PCS }\end{array}$ & NA & NA & NA & NA \\
\hline \multirow[t]{2}{*}{$\begin{array}{l}\text { Mease, et al. } \\
2010\end{array}$} & \multirow[t]{2}{*}{$\begin{array}{l}\text { ETN 25mg BIW } \\
\text { vs. PCB } \\
(\mathrm{N}=205)\end{array}$} & \multirow[t]{2}{*}{$\begin{array}{c}\text { ACR20/ } \\
\text { Week } 12 \\
\text { Week } 24 \text { is } \\
\text { the end of } \\
\text { double- } \\
\text { blind phase }\end{array}$} & $\begin{array}{l}\text { HAQ- } \\
\text { DI }\end{array}$ & $\begin{array}{c}\text { Mean }[\mathrm{SE}] \\
\text { ETN: } 1.1[0.1] \\
\text { PCB: } 1.1[0.1]\end{array}$ & $\begin{array}{c}\text { Week } 4: \\
\text { ETN: } 35.1 \% \Delta \text { from BL } \\
\text { PCB: } 8.0 \% \Delta \text { from BL } \\
\text { Week 12: } \\
\text { ETN: } 53.5 \% \Delta \text { from BL } \\
\text { PCB: } 6.3 \% \Delta \text { from BL } \\
\text { Endpoint score at Week } 24 \\
\text { Mean [SE] } \\
\text { ETN: } 0.5[0.1] \\
\text { PCB: } 1.0[0.1] \\
\\
\text { Calculated } \Delta \text { sores (pooled SD) at } \\
\text { Week 24 } \\
\text { ETN: }-0.6(1.005) \\
\text { PCB: }-0.1(1.02)\end{array}$ & $\begin{array}{c}\text { Week 4: } \\
<0.001 \text { for } \\
\% \Delta \text { from BL } \\
\text { Week } 12 \text { : } \\
<0.001 \text { for } \\
\% \Delta \text { from BL } \\
\text { Week } 24 \text { : } \\
<0.001\end{array}$ & $\begin{array}{l}\text { Effect size for Week 12: } \\
\text { Similar to that at Week } 24 \text { as per } \\
\text { graph in paper } \\
E_{2} \text { at Week } 24 \\
\text { (end of double-blind phase): } \\
\text { Etanercept }=-0.597 \\
\text { PCB }=-0.098\end{array}$ \\
\hline & & & $\begin{array}{l}\text { SF-36 } \\
\text { PCS }\end{array}$ & $\begin{array}{c}\text { Mean [SE] } \\
\text { ETN: } 35.8[1.0] \\
\text { PCB: } 35.7[0.9]\end{array}$ & $\begin{array}{c}\text { Week 4: } \Delta \text { from BL } \\
\text { ETN: } 5.8 \\
\text { PCB: } 0.5 \\
\text { Week 12: } \\
\text { similar to Week 24 as per graph } \\
\text { Endpoint score at Week 24 } \\
\text { Mean [SE] } \\
\text { ETN: } 45.1[1.1] \\
\text { PCB: } 36.4[1.0] \\
\text { Calculated } \Delta \text { sores (pooled SD) at } \\
\text { Week 24 } \\
\text { ETN: } 9.3(10.564) \\
\text { PCB: } 0.7(9.178)\end{array}$ & $\begin{array}{l}\text { Week } 24 \\
<0.001\end{array}$ & $\begin{array}{c}\text { SRM at Week 12: NA } \\
\text { ES }{ }_{2} \text { at Week } 24 \\
\text { (end of double-blind phase): } \\
\text { ETN }=0.880 \\
\text { PCB }=0.073\end{array}$ \\
\hline $\begin{array}{l}\text { Gniadecki, et } \\
\text { al. } 2012 \\
\text { (PRESTA) }\end{array}$ & $\begin{array}{l}\text { ETN 50mg } \\
\text { BIW/QW } \\
\text { vs. }\end{array}$ & $\begin{array}{l}\text { Psoriasis } \\
\text { clear or } \\
\text { almost } \\
\text { clear/ }\end{array}$ & $\begin{array}{l}\text { HAQ- } \\
\text { DI }\end{array}$ & $\begin{array}{l}\text { ETN BIW/QW: } 0.90(0.69) \\
\text { ETN QW/QW: } 0.93(0.70)\end{array}$ & $\begin{array}{l}\text { Endpoint score at Week 24 } \\
\text { ETN BIW/QW: } 0.48(0.60) \\
\text { ETN QW/QW: } 0.49(0.57)\end{array}$ & 0.792 & $\begin{array}{c}\mathrm{ES}_{2} \text { at Week 12: } \\
\text { ETN 50mg BIW/QW: }-0.74 \\
\text { ETN 50mg QW/QW: }-0.69\end{array}$ \\
\hline
\end{tabular}




\begin{tabular}{|c|c|c|c|c|c|c|c|}
\hline & $\begin{array}{l}\text { ETN 50mg } \\
\text { QW/QW } \\
(\mathrm{N}=752)\end{array}$ & week 12 & & & $\begin{array}{c}\text { Calculated } \Delta \text { sores }(\text { pooled SD) } \\
\text { ETN BIW/QW: } \\
-0.42(0.65) \\
\text { ETN QW/QW: }-0.44(0.64)\end{array}$ & & \\
\hline & & & $\begin{array}{l}\text { SF-36 } \\
\text { PCS or } \\
\text { PF }\end{array}$ & NA & NA & NA & NA \\
\hline \multirow[t]{2}{*}{$\begin{array}{l}\text { Mease, et al. } \\
2019 \\
\text { (SEAM-PsA) }\end{array}$} & \multirow[t]{2}{*}{$\begin{array}{l}\text { ETN 50mg QW } \\
\text { plus MTX } \\
\text { vs. } \\
\text { ETN 50mg QW } \\
\text { vs. } \\
\text { MTX alone } \\
(\mathrm{N}=851)\end{array}$} & \multirow[t]{2}{*}{$\begin{array}{l}\text { ACR20/ } \\
\text { Week 24 }\end{array}$} & $\begin{array}{l}\text { HAQ- } \\
\text { DI }\end{array}$ & $\begin{array}{c}\text { Mean [SE] (Calculated SD) } \\
\text { ETN/MTX: } 1.15[0.04] \\
(0.672) \\
\text { ETN: } 1.15[0.04](0.674) \\
\text { MTX: } 1.27[0.04](0.673)\end{array}$ & $\begin{array}{c}\Delta \text { score } \\
\text { Mean [SE] (Calculated SD) } \\
\text { ETN plus MTX: }-0.47[0.04] \\
(0.641) \\
\text { ETN: }-0.44[0.04](0.642) \\
\text { MTX: }-0.41[0.04](0.635)\end{array}$ & $\begin{array}{c}0.67 \text { for ETN } \\
0.34 \text { for } \\
\text { ETN/MTX }\end{array}$ & $\begin{array}{c}\text { SRM at Week 24: } \\
\text { ETN }=-0.733 \\
\text { ETN plus MTX }=-0.685 \\
\text { MTX alone }=-0.646\end{array}$ \\
\hline & & & $\begin{array}{l}\text { SF36 } \\
\text { PCS }\end{array}$ & $\begin{array}{c}\text { Mean [SE] (Calculated SD) } \\
\text { ETN/MTX: } 37.4[0.6] \\
(10.058) \\
\text { ETN: } 37.8[0.5](8.426) \\
\text { MTX: } 35.6[0.5](8.396) \\
\end{array}$ & $\begin{array}{c}\Delta \text { score } \\
\text { Mean [SE] (Calculated SD) } \\
\text { ETN/MTX: } 8.0[0.6](9.619) \\
\text { ETN: } 7.8[0.6](9.60) \\
\text { MTX: } 6.0[0.6](9.544)\end{array}$ & $\begin{array}{c}0.033 \text { for ETN } \\
0.015 \text { for } \\
\text { ETN/MTX }\end{array}$ & $\begin{array}{c}\text { SRM at Week 24: } \\
\text { ETN }=0.832 \\
\text { ETN plus MTX }=0.813 \\
\text { MTX alone }=0.629\end{array}$ \\
\hline \multirow[t]{3}{*}{$\begin{array}{l}\text { Kavanaugh, et } \\
\text { al. } 2009 \\
\text { (GO- } \\
\text { REVEAL) }\end{array}$} & \multirow[t]{3}{*}{$\begin{array}{l}\text { GOL }(2 \text { doses }) \\
\text { vs. PCB } \\
(\mathrm{N}=405)\end{array}$} & \multirow[t]{3}{*}{$\begin{array}{l}\text { ACR20/ } \\
\text { Week } 14\end{array}$} & $\begin{array}{l}\text { HAQ- } \\
\text { DI }\end{array}$ & $\begin{array}{l}\text { GOL 100mg: } 1.05(0.62) \\
\text { GOL 50mg: } 0.98(0.65) \\
\text { PCB: } 1.03(0.55)\end{array}$ & $\begin{array}{l}\Delta \text { score at Week 14: } \\
\text { GOL 100mg: }-0.38(0.51) \\
\text { GOL 50mg: }-0.31(0.50) \\
\text { PCB: }-0.04(0.44)\end{array}$ & $<0.001$ & $\begin{array}{l}\text { SRM at Week 14: } \\
\text { GOL 100mg = -0.75 } \\
\text { GOL 50mg = }-0.62 \\
\text { PCB }=-0.09\end{array}$ \\
\hline & & & & & $\begin{array}{l}\Delta \text { score at Week } 24^{\pi} \\
\text { GOL 100mg: }-0.39(0.50) \\
\text { GOL 50mg: }-0.33(0.55) \\
\text { PCB: } 0.01(0.49)\end{array}$ & & $\begin{array}{c}\text { SRM at Week } 24^{*}: \\
\text { GOL 100mg }=-0.78 \\
\text { GOL 50mg }=-0.60 \\
\text { PCB }=0.02\end{array}$ \\
\hline & & & $\begin{array}{l}\text { SF36 } \\
\text { PCS }\end{array}$ & $\begin{array}{l}\text { GOL 100mg: } 32.79(8.90) \\
\text { GOL 50mg: } 33.03(10.68) \\
\text { PCB: } 31.93(9.25)\end{array}$ & $\begin{array}{c}\Delta \text { score } \\
\text { GOL 100mg: } 7.85(9.55) \\
\text { GOL 50mg: } 6.53(8.88) \\
\text { PCB: } 0.63(7.68)\end{array}$ & $<0.001$ & $\begin{array}{c}\text { SRM at Week 14: } \\
\text { GOL } 100 \mathrm{mg}=0.82 \\
\text { GOL } 50 \mathrm{mg}=0.74 \\
\text { PCB }=0.08\end{array}$ \\
\hline \multirow{2}{*}{$\begin{array}{l}\text { Kavanaugh, et } \\
\text { al. } 2017 \\
\text { (GO- } \\
\text { VIBRANT) }\end{array}$} & \multirow[t]{2}{*}{$\begin{array}{l}\text { GOL ivi } 2 \mathrm{mg} / \mathrm{kg} \\
\text { vs. PCB } \\
(\mathrm{N}=480)\end{array}$} & \multirow[t]{2}{*}{$\begin{array}{l}\text { ACR20/ } \\
\text { Week 14 }\end{array}$} & $\begin{array}{l}\text { HAQ- } \\
\text { DI }\end{array}$ & $\begin{array}{l}\text { GOL: } 1.3(0.6) \\
\text { PCB: } 1.3(0.6)\end{array}$ & $\begin{array}{c}\frac{\Delta \text { score }}{\text { GOL: }-0.60(0.53)} \\
\text { PCB: }-0.12(0.47)\end{array}$ & $<0.001$ & $\begin{array}{c}\text { SRM at Week 14: } \\
\text { GOL ivi }=-1.13 \\
\text { PCB }=-0.26\end{array}$ \\
\hline & & & $\begin{array}{l}\text { SF-36 } \\
\text { PCS }\end{array}$ & $\begin{array}{l}\text { GOL: } 33.1(6.9) \\
\text { PCB: } 34.0(7.2)\end{array}$ & $\begin{array}{c}\Delta \text { score } \\
\text { GOL: } 8.7(7.6) \\
\text { PCB: } 2.7(5.9)\end{array}$ & $<0.001$ & $\begin{array}{c}\text { SRM at Week 14: } \\
\text { GOL ivi }=1.14 \\
\text { PCB }=0.46\end{array}$ \\
\hline $\begin{array}{l}\text { Gladman, et } \\
\text { al. } 2014 \\
\text { (RAPID-PsA) }\end{array}$ & $\begin{array}{l}\text { CZP (2 doses }) \\
\text { vs. PCB } \\
(\mathrm{N}=409) \\
\end{array}$ & $\begin{array}{l}\text { ACR20, } \\
\text { EULAR }\end{array}$ & $\begin{array}{l}\text { HAQ- } \\
\text { DI }\end{array}$ & $\begin{array}{l}\text { CZP 400 Q4W: } 1.3(0.6) \\
\text { CZP 200 Q2W: } 1.3(0.7) \\
\text { PCB: } 1.3(0.7) \\
\end{array}$ & $\begin{array}{c}\stackrel{\Delta \text { score }}{ } \\
\text { CZP 400 Q4W: }-0.39(0.47) \\
\text { CZP 200 Q2W: }-0.45(0.56)\end{array}$ & $<0.001$ & $\begin{array}{c}\text { SRM at Week 12: } \\
\text { CZP 400mg Q4W = }-0.83 \\
\text { CZP 200mg Q2W }=-0.80\end{array}$ \\
\hline
\end{tabular}




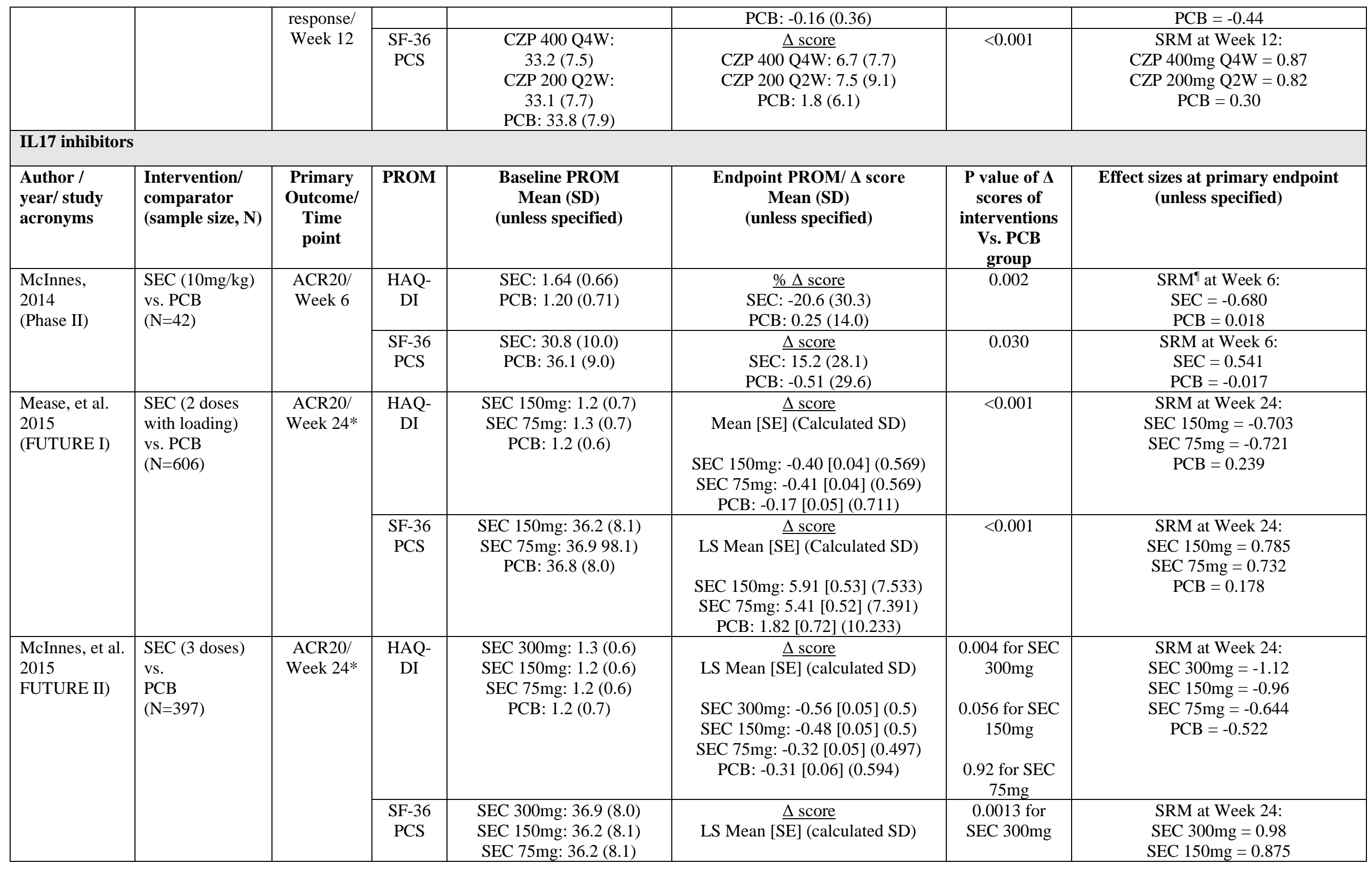




\begin{tabular}{|c|c|c|c|c|c|c|c|}
\hline & & & & PCB: 37.4 (8.8) & $\begin{array}{l}\text { SEC300mg: } 7.25[0.74](7.40) \\
\text { SEC150mg: } 6.39[0.73](7.30) \\
\text { SEC75mg: } 4.38[0.75](7.462) \\
\text { PCB: } 1.95[0.97](9.603)\end{array}$ & $\begin{array}{l}0.0057 \text { for } \\
\text { SEC } 150 \mathrm{mg} \\
0.6421 \text { for } \\
\text { SEC } 75 \mathrm{mg}\end{array}$ & $\begin{array}{c}\text { SEC 75mg }=0.587 \\
\text { PCB }=0.203\end{array}$ \\
\hline \multirow[t]{2}{*}{$\begin{array}{l}\text { Kavanaugh, et } \\
\text { al. } 2016 . \\
\text { (FUTURE II) } \\
\text {-subgroup } \\
\text { analysis }\end{array}$} & \multirow[t]{2}{*}{$\begin{array}{l}\text { TNFi naïve } \\
\text { vs. TNFi } \\
\text { exposed }\end{array}$} & \multirow[t]{2}{*}{$\begin{array}{c}\text { ACR20/ } \\
\text { Week 24* }\end{array}$} & $\begin{array}{c}\text { HAQ- } \\
\text { DI }\end{array}$ & $\begin{array}{l}\text { TNFi naïve: } \\
\text { Pooled SEC: } 1.2(0.6) \\
\text { PCB: } 1.2(0.7) \\
\text { TNFi exposed: } \\
\text { Pooled SEC: } 1.3(0.6) \\
\text { PCB: } 1.1(0.7)\end{array}$ & $\begin{array}{c}\underline{\Delta \text { score }} \\
\text { LS Mean [SE] (calculated SD) } \\
\text { TNFi naïve: } \\
\text { SEC 300mg: }-0.59[0.06](0.49) \\
\text { SEC 150mg: }-0.55[0.06](0.48) \\
\text { SEC 75mg: }-0.37[0.06](0.48) \\
\text { PCB: }-0.35[0.07](0.56) \\
\text { TNFi exposed: } \\
\text { SEC 300mg: }-0.53[0.09](0.52) \\
\text { SEC 150mg: }-0.35[0.08](0.49) \\
\text { SEC 75mg: }-0.23[0.09](0.52) \\
\text { PCB: }-0.23[0.11](0.65)\end{array}$ & $\begin{array}{c}\frac{\text { TNFi naïve: }}{<0.05 \text { for }} \\
\text { 300mg and } \\
150 \mathrm{mg} \\
\\
\frac{\text { TNFi exposed: }}{<0.05 \text { for }} \\
300 \mathrm{mg} \\
\text { NS for } 150 \mathrm{mg}\end{array}$ & $\begin{array}{c}\text { SRM at Week } 24 \\
\text { (TNFi naïve vs. exposed): } \\
\text { SEC 300mg: }-1.20 \text { vs. }-1.02 \\
\text { SEC 150mg: }-1.15 \text { vs. }-0.71 \\
\text { SEC 75mg: }-0.77 \text { vs. }-0.44 \\
\text { PCB: }-0.63 \text { vs }-0.35\end{array}$ \\
\hline & & & $\begin{array}{l}\text { SF-36 } \\
\text { PCS }\end{array}$ & & $\begin{array}{c}\Delta \Delta \text { score } \\
\text { LS Mean [SE] (calculated SD) } \\
\text { TNFi naïve: } \\
\text { SEC 300mg: } 8.05[0.92](7.53) \\
\text { SEC 150mg: } 7.91[0.93](7.38) \\
\text { SEC 75mg: } 5.37[0.94](7.58) \\
\text { PCB: } 2.08 \text { [1.20] (9.52) } \\
\text { TNFi exposed: } \\
\text { SEC 300mg: } 6.56[1.20](6.89) \\
\text { SEC 150mg: } 4.21[1.15](6.995) \\
\text { SEC 75mg: } 3.15[1.20](7.00) \\
\text { PCB: } 2.65[1.66](9.82)\end{array}$ & $\begin{array}{c}\text { TNFi naïve: } \\
<0.0001 \text { for } \\
300 \mathrm{mg} \\
<0.001 \text { for } \\
150 \mathrm{mg} \\
<0.05 \text { for } \\
75 \mathrm{mg} \\
\\
\text { TNFi exposed: } \\
\text { NS for all } \\
\text { doses }\end{array}$ & $\begin{array}{c}\text { SRM at Week 24: } \\
\text { (TNFi naïve Vs. exposed): } \\
\text { SEC 300mg: } 1.07 \text { Vs. } 0.95 \\
\text { SEC 150mg: } 1.07 \text { Vs. } 0.60 \\
\text { SEC 75mg: } 0.71 \text { Vs. } 0.45 \\
\text { PCB: } 0.22 \text { Vs. } 0.27\end{array}$ \\
\hline \multirow[t]{2}{*}{$\begin{array}{l}\text { Nash, et al. } \\
2018 \\
\text { (FUTURE III) }\end{array}$} & \multirow[t]{2}{*}{$\begin{array}{l}\text { SEC (2 doses }) \\
\text { vs. PCB } \\
(\mathrm{N}=414)\end{array}$} & \multirow[t]{2}{*}{$\begin{array}{c}\text { ACR20/ } \\
\text { Week 24* }\end{array}$} & $\begin{array}{l}\text { HAQ- } \\
\text { DI }\end{array}$ & $\begin{array}{l}\text { SEC 300mg: } 1.1(0.7) \\
\text { SEC 150mg: } 1.2(0.6) \\
\text { PCB: } 1.2(0.6)\end{array}$ & $\begin{array}{c}\underline{\Delta \text { score }} \\
\text { Mean [SE] (calculated SD) } \\
\text { SEC 300mg: }-0.38[0.04](0.47) \\
\text { SEC 150mg: }-0.27[0.04](0.47) \\
\text { PCB: }-0.17[0.06](0.70)\end{array}$ & $<0.01$ & $\begin{array}{c}\text { SRM at week 24: } \\
\text { SEC 300mg = -0.81 } \\
\text { SEC } 150 \mathrm{mg}=-0.57 \\
\text { PCB }=-0.24\end{array}$ \\
\hline & & & $\begin{array}{l}\text { SF-36 } \\
\text { PCS }\end{array}$ & $\begin{array}{l}\text { SEC 300mg: } 39.2(8.4) \\
\text { SEC 150mg: } 37.9(7.6)\end{array}$ & $\frac{\Delta \text { score }}{\text { Mean [SE] (calculated SD) }}$ & $<0.01$ & $\begin{array}{l}\text { SRM at week 24: } \\
\text { SEC } 300 \mathrm{mg}=0.93\end{array}$ \\
\hline
\end{tabular}




\begin{tabular}{|c|c|c|c|c|c|c|c|}
\hline & & & & PCB: $37.4(8.5)$ & $\begin{array}{c}\text { SEC 300mg: } 6.46[0.59](6.96) \\
\text { SEC 150mg: } 3.42[0.60](7.05) \\
\text { PCB: } 2.94[0.83](9.71)\end{array}$ & & $\begin{array}{c}\text { SEC 150mg }=0.49 \\
\text { PCB }=0.30\end{array}$ \\
\hline \multirow[t]{2}{*}{$\begin{array}{l}\text { Mease, et al. } \\
2017 \\
\text { (SPIRIT-P1) }\end{array}$} & \multirow[t]{2}{*}{$\begin{array}{l}\text { IXE }(2 \text { doses }) \\
\text { vs. PCB } \\
\text { vs. ADA } \\
(\mathrm{N}=417)\end{array}$} & \multirow[t]{2}{*}{$\begin{array}{c}\text { ACR20/ } \\
\text { Week 24* }\end{array}$} & $\begin{array}{l}\text { HAQ- } \\
\text { DI }\end{array}$ & $\begin{array}{l}\text { IXE Q2W: } 1.2(0.57) \\
\text { IXE Q4W: } 1.2(0.54) \\
\text { PCB: } 1.2(0.60) \\
\text { ADA: } 1.1(0.59)\end{array}$ & $\begin{array}{c}\Delta \text { score at Week 12 } \\
\text { LS Mean [SE] (calculated SD) } \\
\text { IXE Q2W: }-0.47[0.05](0.51) \\
\text { IXE Q4W: }-0.37[0.05](0.52) \\
\text { PCB: }-0.13[0.05](0.51) \\
\text { ADA: }-0.35[0.05](0.50) \\
\\
\Delta \text { score at Week } 24 \\
\text { (primary endpoint) } \\
\text { LS Mean [SE] (calculated SD) } \\
\text { IXE Q2W: }-0.50[0.05](0.51) \\
\text { IXE Q4W: }-0.44[0.05](0.52) \\
\text { PCB: }-0.18[0.05](0.51) \\
\text { ADA: }-0.37[0.05](0.50)\end{array}$ & $\begin{array}{c}\text { Week } 12 \\
<0.001 \text { for } \\
\text { IXE } \\
\text { Q4W/Q2W } \\
<0.01 \text { for } \\
\text { ADA } \\
\text { Week } 24 \\
<0.001 \text { for } \\
\text { IXE } \\
\text { Q4W/Q2W } \\
<0.01 \text { for } \\
\text { ADA }\end{array}$ & $\begin{array}{c}\text { SRM at Week 12: } \\
\text { IXE Q2W = -0.92 } \\
\text { IXE Q4W =-0.71 } \\
\text { PCB }=-0.25 \\
\text { ADA }=-0.70 \\
\\
\text { SRM at Week 24: } \\
\text { (primary end-point) } \\
\text { IXE Q2W = -0.98 } \\
\text { IXE Q4W = -0.85 } \\
\text { PCB }=-0.35 \\
\text { ADA }=-0.74\end{array}$ \\
\hline & & & $\begin{array}{c}\text { SF-36 } \\
\text { PCS }\end{array}$ & $\begin{array}{c}\text { IXE Q2W: } 34.2(8.7) \\
\text { IXE Q4W: } 32.4(10.1) \\
\text { PCB: } 34.0(8.3) \\
\text { ADA: } 33.9(8.8)\end{array}$ & $\begin{array}{c}\Delta \text { score at Week 12 } \\
\text { LS Mean [SE] (calculated SD) } \\
\text { IXE Q2W: } 7.6[0.8](4.08) \\
\text { IXE Q4W: } 5.8[0.8](5.00) \\
\text { PCB: } 2.3[0.8](4.23) \\
\text { ADA: } 5.7[0.8](3.39) \\
\\
\Delta \text { score at Week 24 } \\
\text { (primary endpoint) } \\
\text { LS Mean [SE] (calculated SD) } \\
\text { IXE Q2W: } 8.2[0.9](4.59) \\
\text { IXE Q4W: } 7.5[0.9](5.62) \\
\text { PCB: } 2.9[1.0](5.29) \\
\text { ADA: } 6.8[0.9](3.82)\end{array}$ & $\begin{array}{c}\text { Week } 12 \\
<0.001 \text { for } \\
\text { IXE } \\
\text { Q4W/Q2W } \\
<0.01 \text { for } \\
\text { ADA } \\
\text { Week } 24 \\
\text { IXE } \\
\text { Q4W/Q2W: } \\
<0.001 \\
\text { ADA: }<0.01\end{array}$ & $\begin{array}{c}\text { SRM at Week 12: } \\
\text { IXE Q2W = 1.86 } \\
\text { IXE Q4W = 1.16 } \\
\text { PCB }=0.54 \\
\text { ADA }=1.68 \\
\\
\text { SRM at Week 24: } \\
\text { (primary endpoint) } \\
\text { IXE Q2W = 1.79 } \\
\text { IXE Q4W = } 1.33 \\
\text { PCB }=0.55 \\
\text { ADA }=1.78\end{array}$ \\
\hline $\begin{array}{l}\text { Nash, et al. } \\
2017 \\
\text { (SPIRIT-P2) }\end{array}$ & $\begin{array}{l}\text { IXE ( } 2 \text { doses }) \\
\text { vs. PCB } \\
(\mathrm{N}=363)\end{array}$ & $\begin{array}{c}\text { ACR20/ } \\
\text { Week 24* }\end{array}$ & $\begin{array}{l}\text { HAQ- } \\
\text { DI }\end{array}$ & $\begin{array}{l}\text { IXE Q2W: } 1.2(0.6) \\
\text { IXE Q4W: } 1.2(0.6) \\
\text { PCB: } 1.2(0.7)\end{array}$ & $\begin{array}{c}\frac{\Delta \text { score }}{2} \\
\text { LS Mean [SE] (calculated SD) } \\
\text { IXE Q2W: }-0.4[0.1](1.11) \\
\text { IXE Q4W: }-0.6[0.1](1.10) \\
\text { PCB: }-0.2[0.1](1.09)\end{array}$ & $\begin{array}{l}0.0002 \text { for } \\
\text { IXE Q2W } \\
<0.0001 \text { for } \\
\text { IXE Q4W }\end{array}$ & $\begin{array}{c}\text { SRM at Week 24: } \\
\text { IXE Q2W = -0.36 } \\
\text { IXE Q4W = -0.55 } \\
\text { PCB }=-0.18\end{array}$ \\
\hline
\end{tabular}




\begin{tabular}{|c|c|c|c|c|c|c|c|}
\hline & & & $\begin{array}{l}\text { SF-36 } \\
\text { PCS }\end{array}$ & $\begin{array}{c}\text { IXE Q2W: } 34.3(9.1) \\
\text { IXE Q4W: } 34.8(8.8) \\
\text { PCB: } 33.9(9.0)\end{array}$ & $\begin{array}{c}\underline{\Delta \text { score }} \\
\text { LS Mean [SE] (calculated SD) } \\
\text { IXE Q2W: } 4.0[1.2](13.31) \\
\text { IXE Q4W: } 3.6[1.2](13.25) \\
\text { PCB: } 0.9[1.3](14.12)\end{array}$ & $\begin{array}{c}0.009 \text { for IXE } \\
\text { Q2W } \\
0.02 \text { for IXE } \\
\text { Q4W }\end{array}$ & $\begin{array}{c}\text { SRM at Week 24: } \\
\text { IXE Q2W }=0.30 \\
\text { IXE Q4W }=0.27 \\
\text { PCB }=0.06\end{array}$ \\
\hline \multirow[t]{2}{*}{$\begin{array}{l}\text { Mease, et al. } \\
2014 \\
\text { Phase II }\end{array}$} & \multirow[t]{2}{*}{$\begin{array}{l}\text { BRO (2 doses) } \\
\text { vs. PCB } \\
(\mathrm{N}=168)\end{array}$} & \multirow[t]{2}{*}{$\begin{array}{l}\text { ACR20/ } \\
\text { Week 12 }\end{array}$} & $\begin{array}{c}\text { HAQ- } \\
\text { DI }\end{array}$ & $\begin{array}{l}\text { BRO 280mg: } 1.4(0.6) \\
\text { BRO 140mg: } 1.2(0.7) \\
\text { PCB: } 1.3(0.6)\end{array}$ & $\begin{array}{c}\frac{\% \Delta \text { Median }[\mathrm{IQR}]}{280 \mathrm{mg}:-13.3[-36.4,0.0]} \\
\text { BRO } \\
\text { BRO 140mg -6.5 [-45.0,6.9) } \\
\text { PCB: }-7.7[-23.1,13.3] \\
\text { Calculated mean (SD) } \\
\text { BRO 280mg: }-16.57(27.70) \\
\text { BRO 140mg -14.87 (39.47) } \\
\text { PCB: }-5.83(27.71)\end{array}$ & $\begin{array}{c}\text { NS for all } \\
\text { doses }\end{array}$ & $\begin{array}{c}\mathrm{SRM}^{\ddagger} \text { at Week 12: } \\
\text { BRO 280mg = }-0.60 \\
\text { BRO 140mg }=-0.38 \\
\text { PCB }=-0.21\end{array}$ \\
\hline & & & $\begin{array}{l}\text { SF-36 } \\
\text { PCS }\end{array}$ & NA & $\begin{array}{l}\Delta \text { score } \\
\text { BRO 280mg: } 3.0 \\
\text { BRO 140mg: } 2.0 \\
\text { PCB: } 0.7\end{array}$ & $\begin{array}{c}<0.05 \text { for } \\
280 \mathrm{mg} \\
\text { NS for } 140 \mathrm{mg}\end{array}$ & $\begin{array}{l}\text { Difference from PCB (95\% CI): } \\
\text { BRO 280mg: } 2.4 \text { (0.1 to 4.6) } \\
\text { BRO 140mg: } 1.4 \text { (-0.8 to 3.6) } \\
\text { Insufficient data for effect size } \\
\text { calculation }\end{array}$ \\
\hline \multicolumn{8}{|l|}{ IL12 inhibitors } \\
\hline $\begin{array}{l}\text { Author / } \\
\text { year/ study } \\
\text { acronyms }\end{array}$ & $\begin{array}{l}\text { Intervention/ } \\
\text { comparison } \\
\text { (sample size) }\end{array}$ & $\begin{array}{c}\text { Primary } \\
\text { Outcome/ } \\
\text { Time } \\
\text { point }\end{array}$ & PROM & $\begin{array}{c}\text { Baseline PROM } \\
\text { Mean (SD) } \\
\text { (unless specified) }\end{array}$ & $\begin{array}{c}\text { Endpoint PROM/ } \Delta \text { score } \\
\text { Mean (SD) } \\
\text { (unless specified) }\end{array}$ & $\begin{array}{c}\text { P value of } \Delta \\
\text { scores of } \\
\text { interventions } \\
\text { Vs. PCB } \\
\text { group }\end{array}$ & $\begin{array}{c}\text { Effect sizes at primary endpoint } \\
\text { (unless specified) }\end{array}$ \\
\hline \multirow[t]{2}{*}{$\begin{array}{l}\text { Gottlieb, et al. } \\
2009\end{array}$} & $\begin{array}{l}\text { UST (2 doses }) \\
\text { vs. PCB } \\
(\mathrm{N}=146)\end{array}$ & $\begin{array}{l}\text { ACR20/ } \\
\text { Week 12 }\end{array}$ & $\begin{array}{l}\text { HAQ- } \\
\text { DI }\end{array}$ & $\begin{array}{c}\text { O-12 Week } \\
\text { Median }[\mathrm{IQR}] \\
\text { UST: } 0.9[0.5,1.4] \\
\text { PCB: } 0.8[0.3,1.1]\end{array}$ & $\begin{array}{c}\underline{\Delta \text { score }} \\
\text { Median }[\mathrm{IQR}] \\
\text { UST: }-0.25[-0.5,0.0] \\
\text { PCB: } 0.0[-0.25,0.13] \\
\\
\text { Calculated mean (SD) } \\
\text { UST: }-0.25(0.38) \\
\text { PCB: }-0.04(0.29)\end{array}$ & 0.0005 & $\begin{array}{c}\text { SRM }^{¥} \text { at Week 12: } \\
\text { UST }=-0.66 \\
\text { PCB }=-0.14\end{array}$ \\
\hline & & & $\begin{array}{l}\text { SF-36 } \\
\text { PCS or } \\
\text { PF }\end{array}$ & NA & NA & NA & NA \\
\hline
\end{tabular}




\begin{tabular}{|c|c|c|c|c|c|c|c|}
\hline & & /Week 24* & & & $\begin{array}{l}\text { PCB: } 0.00[-0.38,0.13] \\
\text { Calculated mean (SD) } \\
\text { UST 90mg: }-0.33(0.56) \\
\text { UST 45mg: }-0.29(0.47) \\
\text { PCB: }-0.08(0.38)\end{array}$ & & \\
\hline & & & $\begin{array}{c}\text { SF-36 } \\
\text { PCS }\end{array}$ & $\begin{array}{c}\text { Median [IQR] } \\
\text { UST 90mg: } 36.5[30.2,40.1] \\
\text { UST 45mg: } 35.5[30.6,40.1] \\
\text { PCB: } 35.8[31.8,40.1]\end{array}$ & $\begin{array}{c}\Delta \text { score } \\
\text { Median [IQR }] \\
\text { UST 90mg: } 5.8[0.6,10.9] \\
\text { UST 45mg: } 3.9[-1.3,10.7] \\
\text { PCB: } 1.2[-2.3,5.2] \\
\\
\text { Calculated mean (SD) } \\
\text { UST 90mg: } 5.77(7.69) \\
\text { UST 45mg: } 4.33(8.96) \\
\text { PCB: } 1.37(5.60)\end{array}$ & $\begin{array}{c}<0.0001 \text { for } \\
\text { both UST } \\
\text { doses: }\end{array}$ & $\begin{array}{c}\text { SRM }^{\mp} \text { at Week 24: } \\
\text { UST 90mg }=0.75 \\
\text { UST 45mg }=0.48 \\
\text { PCB }=0.24\end{array}$ \\
\hline $\begin{array}{l}\text { Ritchlin, et al. } \\
2014 \\
\text { (PSUMMIT } \\
\text { II) }\end{array}$ & $\begin{array}{l}\text { UST } \\
(2 \text { doses }) \\
\text { vs. PCB } \\
(\mathrm{N}=312)\end{array}$ & $\begin{array}{c}\text { ACR20, } \\
\text { EULAR } \\
\text { response } \\
\text { PASI75/ } \\
\text { Week 24* }\end{array}$ & $\begin{array}{c}\text { HAQ- } \\
\text { DI }\end{array}$ & $\begin{array}{c}\text { Median [IQR] } \\
\text { UST 90mg: } 1.3[0.8,1.9] \\
\text { UST 45mg: } 1.4[0.8,1.9] \\
\text { PCB: } 1.3[0.8,1.8]\end{array}$ & $\begin{array}{c}\Delta \text { score } \\
\text { Median [IQR] } \\
\text { UST 90mg: }-0.25[-0.50,0.00] \\
\text { UST 45mg: }-0.13[-0.38,0.00] \\
\text { PCB: } 0.00[-0.13,0.13] \\
\text { Calculated mean (SD) } \\
\text { UST 90mg: }-0.25(0.38) \\
\text { UST 45mg: }-0.17(0.29) \\
\text { PCB: } 0.00(0.38) \\
\text { TNFi naïve } \\
\text { Median [IQR }] \\
\text { UST 90mg: }-0.25[-0.50,0.00] \\
\text { UST 45mg: -0.25 [-0.50, } 0.00] \\
\text { PCB: 0.00 [-0.25, 0.25] } \\
\text { Calculated mean (SD) } \\
\text { UST 90mg: }-0.25(0.38) \\
\text { UST 45mg: }-0.25(0.38) \\
\text { PCB: } 0.00(0.38) \\
\text { TNFi exposed } \\
\text { Median [IQR] } \\
\text { UST 90mg: }-0.19[-0.38,0.00] \\
\text { UST 45mg: }-0.13[-0.38,0.00]\end{array}$ & $\begin{array}{l}<0.001 \text { for } \\
\text { UST } 90 \mathrm{mg} \\
<0.01 \text { for UST } \\
\quad 45 \mathrm{mg}\end{array}$ & $\begin{array}{c}\text { SRM }^{¥} \text { at Week } 24: \\
\text { UST 90mg }=-0.66 \\
\text { UST } 45 \mathrm{mg}=-0.59 \\
\text { PCB }=0.00 \\
\text { Subgroups analysis: } \\
\text { SRM } \\
\text { (TNFi at Week } 24 \\
\text { UST 90mg }=-0.66 \text { vs. }-0.66 \\
\text { UST 45mg }=-0.66 \text { vs. }-0.59 \\
\text { PCB }=0 \text { vs. } 0\end{array}$ \\
\hline
\end{tabular}




\begin{tabular}{|c|c|c|c|c|c|c|c|}
\hline & & & & & $\begin{array}{l}\text { PCB: } 0.00[-0.13,0.13] \\
\text { Calculated mean (SD) } \\
\text { UST 90mg: }-0.19(0.29) \\
\text { UST 45mg: }-0.17(0.29) \\
\text { PCB: } 0.00(0.20)\end{array}$ & & \\
\hline & & & $\begin{array}{l}\text { SF-36 } \\
\text { PCS }\end{array}$ & $\begin{array}{c}\text { Median [IQR] } \\
\text { UST 90mg: } 28.2[21.8,33.6] \\
\text { UST 45mg: } 28.0[22.6,34.0] \\
\text { PCB: } 29.4[23.3,36.2]\end{array}$ & $\begin{array}{c}\frac{\Delta \text { score }}{\text { Median }[\mathrm{IQR}]} \\
\text { UST 90mg: } 3.5[-0.2,10.1] \\
\text { UST 45mg: } 2.7[-0.7,9.1] \\
\text { PCB: } 0.00[-0.8,4.0] \\
\\
\text { Calculated mean (SD) } \\
\text { UST 90mg: } 4.67(7.75) \\
\text { UST 45mg: } 3.7(7.37) \\
\text { PCB: } 1.07(3.61)\end{array}$ & $\begin{array}{l}<0.01 \text { for both } \\
\text { UST doses }\end{array}$ & $\begin{array}{l}\text { SRM }^{\ddagger} \text { at Week 24: } \\
\text { UST } 90 \mathrm{mg}=0.60 \\
\text { UST } 45 \mathrm{mg}=0.50 \\
\text { PCB }=0.30\end{array}$ \\
\hline \multirow[t]{2}{*}{$\begin{array}{l}\text { Araugo, et al. } \\
2019 \\
\text { (ECLIPSA) }\end{array}$} & \multirow[t]{2}{*}{$\begin{array}{l}\text { UST }(45 \mathrm{mg} \\
\text { or } 100 \mathrm{mg} \text { if } \\
\text { body weight } \\
>100 \mathrm{~kg}) \\
\text { vs. TNFi } \\
(\mathrm{N}=47)\end{array}$} & \multirow[t]{2}{*}{$\begin{array}{l}\text { Enthesitis } \\
\text { clearance } \\
\text { (SPARCC } \\
=0 \text { ) } \\
\text { at Week } 24\end{array}$} & $\begin{array}{l}\text { HAQ- } \\
\text { DI }\end{array}$ & $\begin{array}{l}\text { UST: } 0.87(0.63) \\
\text { TNFi: } 1.17(0.62)\end{array}$ & $\begin{array}{c}\text { Score at Week 24 } \\
\text { Median }[\mathrm{IQR}] \\
\text { UST: } 0[0.25] \\
\text { TNFi: } 0.3[0.35] \\
\text { Calculated } \Delta \text { score (pooled SD) } \\
\text { UST: }-0.87(0.48) \\
\text { TNFi: }-0.87(0.50) \\
\end{array}$ & NA & $\begin{array}{c}\mathrm{ES}_{2}{ }^{¥} \text { at Week 24: } \\
\mathrm{UST}=-1.81 \\
\mathrm{TNFi}=-1.74\end{array}$ \\
\hline & & & $\begin{array}{l}\text { SF-36 } \\
\text { PCS }\end{array}$ & $\begin{array}{l}\text { UST: } 29.1(9.6) \\
\text { TNFi: } 29.5(9.5)\end{array}$ & $\begin{array}{c}\text { Score at Week 24 } \\
\text { Median [IQR] } \\
\text { UST: } 52.8[6.1) \\
\text { TNFi: } 46[11.6] \\
\text { Calculated } \Delta \text { score (pooled SD) } \\
\text { UST: } 23.7(8.04) \\
\text { TNFi: } 16.5(10.60) \\
\end{array}$ & NA & $\begin{array}{c}\mathrm{ES}_{2}{ }^{¥} \text { at Week 24: } \\
\mathrm{UST}=2.95 \\
\mathrm{TNFi}=1.56\end{array}$ \\
\hline \multicolumn{8}{|l|}{ IL23 inhibitors } \\
\hline $\begin{array}{l}\text { Author / } \\
\text { year/ study } \\
\text { acronyms }\end{array}$ & $\begin{array}{l}\text { Intervention/ } \\
\text { comparison } \\
\text { (sample size) }\end{array}$ & $\begin{array}{c}\text { Primary } \\
\text { Outcome/ } \\
\text { Time } \\
\text { point }\end{array}$ & PROM & $\begin{array}{c}\text { Baseline PROM } \\
\text { Mean (SD) } \\
\text { (unless specified) }\end{array}$ & $\begin{array}{c}\text { End point PROM/ } \Delta \text { score } \\
\text { Mean (SD) } \\
\text { (unless specified) }\end{array}$ & $\begin{array}{l}\text { P value of } \Delta \\
\text { scores of } \\
\text { intervention } \\
\text { vs. PCB } \\
\text { group }\end{array}$ & $\begin{array}{c}\text { Effect sizes at primary end point } \\
\text { (unless specified) }\end{array}$ \\
\hline $\begin{array}{l}\text { Deodhar, et al. } \\
2018\end{array}$ & $\begin{array}{l}\text { GUS 100mg } \\
\text { vs. PCB }\end{array}$ & $\begin{array}{c}\text { ACR20/ } \\
\text { Week24* }\end{array}$ & $\begin{array}{c}\text { HAQ- } \\
\text { DI }\end{array}$ & $\begin{array}{l}\text { GUS: } 1.4(0.6) \\
\text { PCB: } 1.3(0.5)\end{array}$ & $\begin{array}{c}\Delta \text { score } \\
\text { GUS: }-0.42(0.51)\end{array}$ & 0.00025 & $\begin{array}{c}\text { SRM at Week 24: } \\
\text { GUS }=-0.82\end{array}$ \\
\hline
\end{tabular}




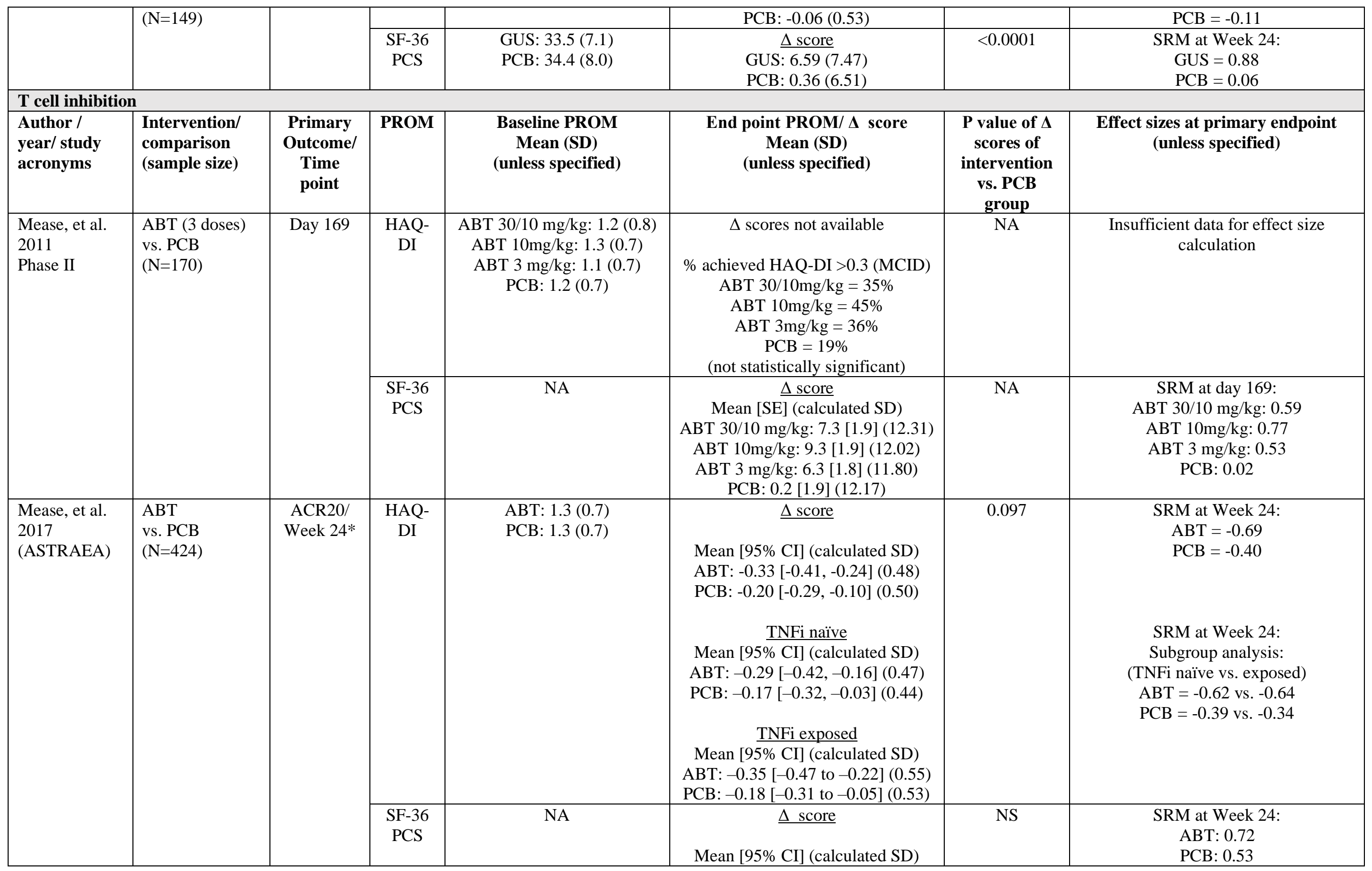




\begin{tabular}{|c|c|c|c|c|c|c|c|}
\hline & & & & & $\begin{array}{l}\text { ABT: } 5.11[3.86,6.36](7.10) \\
\text { PCB: } 3.69[2.30,5.08](6.98)\end{array}$ & & \\
\hline \multirow[t]{3}{*}{$\begin{array}{l}\text { Mease, et al. } \\
2006\end{array}$} & \multirow[t]{3}{*}{$\begin{array}{l}\text { ALC/MTX } \\
\text { vs. PCB/MTX } \\
(\mathrm{N}=185)\end{array}$} & \multirow[t]{3}{*}{$\begin{array}{l}\text { ACR20/ } \\
\text { Week } 12\end{array}$} & $\begin{array}{l}\text { HAQ- } \\
\text { DI }\end{array}$ & $\begin{array}{l}\text { ALC: } 1.0 \\
\text { PCB: } 1.1\end{array}$ & $\begin{array}{l}\underline{\Delta \text { Score }} \\
\text { ALC }-24.3 \\
\text { PCB: }-7.7\end{array}$ & NS & $\begin{array}{c}\% \Delta \text { in HAQ-DI at Week 12: } \\
\text { ALC/MTX }=-24.5 \% \\
\text { PCB/MTX }=-7.7 \% \\
(\mathrm{NS})\end{array}$ \\
\hline & & & & & & & Inadequate data for ES calculations \\
\hline & & & $\begin{array}{l}\text { SF-36 } \\
\text { PCS or } \\
\text { PF }\end{array}$ & NA & NA & NA & NA \\
\hline \multicolumn{8}{|c|}{ JAK inhibitors } \\
\hline $\begin{array}{l}\text { Author / } \\
\text { year/ study } \\
\text { acronyms }\end{array}$ & $\begin{array}{l}\text { Intervention/ } \\
\text { comparator } \\
(\text { sample size, } N)\end{array}$ & $\begin{array}{l}\text { Primary } \\
\text { Outcome/ } \\
\text { Time } \\
\text { point } \\
\end{array}$ & PROM & $\begin{array}{l}\text { Baseline PROM } \\
\text { Mean (SD) } \\
\text { (unless specified) }\end{array}$ & $\begin{array}{c}\text { End point PROM/ } \Delta \text { score } \\
\text { Mean (SD) } \\
\text { (unless specified) }\end{array}$ & $\begin{array}{l}\text { P value of } \Delta \\
\text { scores of } \\
\text { interventions } \\
\text { vs. PCB } \\
\end{array}$ & $\begin{array}{c}\text { Effect sizes at primary endpoint } \\
\text { (unless specified) }\end{array}$ \\
\hline \multirow[t]{2}{*}{$\begin{array}{l}\text { Mease, et al. } \\
2017 \text { (OPAL } \\
\text { Broaden) }\end{array}$} & \multirow[t]{3}{*}{$\begin{array}{l}\text { TOF }(2 \text { doses }) \\
\text { vs. PCB } \\
\text { vs. ADA } \\
(\mathrm{N}=422)\end{array}$} & \multirow[t]{3}{*}{$\begin{array}{l}\text { ACR20/ } \\
3 \text { months }\end{array}$} & $\begin{array}{l}\text { HAQ- } \\
\text { DI }\end{array}$ & $\begin{array}{l}\text { TOF 10mg: } 1.1(0.6) \\
\text { TOF 5mg: } 1.2(0.6) \\
\text { PCB: } 1.1(0.6) \\
\text { ADA: } 1.1(0.6)\end{array}$ & $\begin{array}{c}\underline{\Delta \text { score }} \\
\text { Mean [SE] (calculated SD) } \\
\text { TOF 10mg: }-0.40[0.05](0.51) \\
\text { TOF 5mg: }-0.35[0.05](0.51) \\
\text { PCB: }-0.18[0.05](0.50) \\
\text { ADA: }-0.38[0.05](0.50) \\
\end{array}$ & $\begin{array}{c}<0.001 \text { for } \\
\text { TOF } 10 \mathrm{mg} \\
<0.01 \text { for TOF } \\
\quad 5 \mathrm{mg} \\
\text { NS for ADA } \\
\end{array}$ & $\begin{array}{c}\text { SRM at } 3 \text { month: } \\
\text { TOF 10mg }=-0.78 \\
\text { TOF 5mg }=-0.69 \\
\text { PCB }=-0.36 \\
\text { ADA }=-0.76\end{array}$ \\
\hline & & & $\begin{array}{l}\text { SF-36 } \\
\text { PF }\end{array}$ & NA & $\begin{array}{c}\underline{\Delta \text { score }} \\
\text { Mean [SE] (calculated SD) } \\
\text { TOF 10mg: 5.2 [0.8] (8.12) } \\
\text { TOF 5mg: } 5.2[0.8](8.08) \\
\text { PCB: } 2.1[0.9](9.09) \\
\text { ADA: } 5.2[0.9](9.04)\end{array}$ & All NS & $\begin{array}{c}\text { SRM at } 3 \text { month: } \\
\text { TOF } 10 \mathrm{mg}=0.64 \\
\text { TOF } 5 \mathrm{mg}=0.64 \\
\text { PCB }=0.23 \\
\text { ADA }=0.58\end{array}$ \\
\hline $\begin{array}{l}\text { Strand, et al. } \\
2019 \text { (OPAL } \\
\text { Broaden) }\end{array}$ & & & $\begin{array}{l}\text { SF-36 } \\
\text { PCS }\end{array}$ & $\begin{array}{l}\text { TOF 10mg: } 36.4(7.6) \\
\text { TOF 5mg: } 35.4(7.9) \\
\text { PCB: } 36.0(7.4) \\
\text { ADA: } 35.9(8.6)\end{array}$ & $\begin{array}{c}\Delta \text { score } \\
\text { Mean [SE] (calculated SD) } \\
\text { TOF 10mg: 5.69 [0.74] (7.55) } \\
\text { TOF 5mg: } 5.51[0.73](7.55) \\
\text { PCB: } 2.68[0.79](8.10) \\
\text { ADA: } 6.23[0.75](7.72)\end{array}$ & $\begin{array}{l}<0.01 \text { for both } \\
\text { doses of TOF } \\
<0.001 \text { for } \\
\text { ADA }\end{array}$ & $\begin{array}{c}\text { SRM at } 3 \text { month: } \\
\text { TOF } 10 \mathrm{mg}=0.75 \\
\text { TOF } 5 \mathrm{mg}=0.73 \\
\text { PCB }=0.33 \\
\text { ADA }=0.81\end{array}$ \\
\hline $\begin{array}{l}\text { Gladman, et } \\
\text { al. } \\
2017 \\
\text { (OPAL } \\
\text { Beyond) }\end{array}$ & $\begin{array}{l}\text { TOF }(2 \text { doses }) \\
\text { vs. PCB } \\
(\mathrm{N}=395)\end{array}$ & $\begin{array}{l}\text { ACR20, } \\
\text { in HAQ- } \\
\text { DI/ } \\
3 \text { months }\end{array}$ & $\begin{array}{l}\text { HAQ- } \\
\text { DI }\end{array}$ & $\begin{array}{l}\text { TOF 10mg: } 1.4(0.6) \\
\text { TOF 5mg: } 1.3(0.7) \\
\text { PCB: } 1.3(0.8)\end{array}$ & $\begin{array}{c}\underline{\Delta \text { score }} \\
\text { Mean [SE] (calculated SD) } \\
\text { TOF 10mg: }-0.35[0.05](0.55) \\
\text { TOF 5mg: }-0.39[0.05](0.56) \\
\text { PCB: }-0.14[0.05](0.54) \\
\end{array}$ & $\begin{array}{l}<0.05 \text { for both } \\
\text { doses }\end{array}$ & $\begin{array}{l}\text { SRM at } 3 \text { month: } \\
\text { TOF } 10 \mathrm{mg}=-0.64 \\
\text { TOF } 5 \mathrm{mg}=-0.70 \\
\text { PCB }=-0.26\end{array}$ \\
\hline
\end{tabular}




\begin{tabular}{|c|c|c|c|c|c|c|c|}
\hline & & & $\begin{array}{l}\text { SF-36 } \\
\text { PF }\end{array}$ & $\begin{array}{l}\text { Norm based scores } \\
\text { TOF 10mg: } 32.1(9.9) \\
\text { TOF 5mg: } 33.5(10.4) \\
\text { PCB: } 34.0(11.0)\end{array}$ & $\begin{array}{c}\underline{\Delta \text { score }} \\
\text { Mean [SE] (calculated SD) } \\
\text { TOF 10mg: } 4.1[0.7](7.67) \\
\text { TOF 5mg: } 5.0[0.7](7.79) \\
\text { PCB: } 1.7[0.7](7.57) \\
\end{array}$ & NS & $\begin{array}{l}\text { SRM at } 3 \text { month: } \\
\text { TOF } 10 \mathrm{mg}=0.53 \\
\text { TOF } 5 \mathrm{mg}=0.64 \\
\text { PCB }=0.22\end{array}$ \\
\hline $\begin{array}{l}\text { Strand, et al. } \\
2019 \text { (OPAL } \\
\text { Beyond) }\end{array}$ & & & $\begin{array}{l}\text { SF-36 } \\
\text { PCS }\end{array}$ & $\begin{array}{l}\text { TOF 10mg: } 31.7(8.9) \\
\text { TOF 5mg: } 33.6(8.5) \\
\text { PCB: } 34.7(9.6)\end{array}$ & $\begin{array}{c}\underline{\Delta \text { score }} \\
\text { Mean [SE] (calculated SD) } \\
\text { TOF 10mg: } 5.34[0.69](7.93) \\
\text { TOF 5mg: } 5.18[0.68](7.78) \\
\text { PCB: } 1.77[0.69](7.90) \\
\end{array}$ & $\begin{array}{l}<0.001 \text { for } \\
\text { both doses }\end{array}$ & $\begin{array}{l}\text { SRM at } 3 \text { month: } \\
\text { TOF } 10 \mathrm{mg}=0.67 \\
\text { TOF } 5 \mathrm{mg}=0.67 \\
\text { PCB }=0.22\end{array}$ \\
\hline \multirow{3}{*}{$\begin{array}{l}\text { Mease, et al. } \\
2018 \\
\text { (EQUATOR) } \\
\text { Phase II }\end{array}$} & \multirow[t]{3}{*}{$\begin{array}{l}\text { FIL 200mg } \\
\text { vs. PCB } \\
(\mathrm{N}=131)\end{array}$} & \multirow[t]{3}{*}{$\begin{array}{l}\text { ACR20/ } \\
\text { Week } 16\end{array}$} & $\begin{array}{l}\text { HAQ- } \\
\text { DI }\end{array}$ & $\begin{array}{l}\text { FIL: } 1.43(0.5) \\
\text { PCB: } 1.36(0.6)\end{array}$ & $\begin{array}{l}\text { Score at end point } \\
\text { FIL: } 0.86(0.6) \\
\text { PCB: } 1.09(0.6)\end{array}$ & 0.0009 & $\begin{array}{c}\text { SRM at Week 16: } \\
\text { FIL }=-1.14 \\
\text { PCB }=-0.56\end{array}$ \\
\hline & & & & & $\begin{array}{c}\Delta \text { score } \\
\text { FIL: }-0.57(0.5) \\
\text { PCB: }-0.28(0.5)\end{array}$ & & $\begin{array}{c}\mathrm{ES}_{2} \text { at Week 16: } \\
\mathrm{FIL}=-1.14 \\
\mathrm{PCB}=-0.47\end{array}$ \\
\hline & & & $\begin{array}{l}\text { SF-36 } \\
\text { PF }\end{array}$ & NA & NA & 0.0009 & $\begin{array}{l}\text { Insufficient data for effect size } \\
\text { calculation }\end{array}$ \\
\hline \multicolumn{8}{|l|}{ IL6 inhibitors } \\
\hline $\begin{array}{l}\text { Author / } \\
\text { year/ (study } \\
\text { acronyms) }\end{array}$ & $\begin{array}{l}\text { Intervention/ } \\
\text { comparator } \\
(\text { sample size, } N)\end{array}$ & $\begin{array}{c}\text { Primary } \\
\text { Outcome/ } \\
\text { Time } \\
\text { point }\end{array}$ & PROM & $\begin{array}{c}\text { Baseline PROM } \\
\text { Mean (SD) } \\
\text { (unless specified) }\end{array}$ & $\begin{array}{c}\text { End point PROM/ } \Delta \text { score } \\
\text { Mean (SD) } \\
\text { (unless specified) }\end{array}$ & $\begin{array}{l}\text { P value of } \Delta \\
\text { scores of } \\
\text { intervention } \\
\text { vs. PCB } \\
\text { group } \\
\end{array}$ & $\begin{array}{c}\text { Effect sizes at primary end point } \\
\text { (unless specified) }\end{array}$ \\
\hline \multirow[t]{2}{*}{$\begin{array}{l}\text { Mease, et al. } \\
2016 \\
\text { Phase II }\end{array}$} & \multirow[t]{2}{*}{$\begin{array}{l}\text { CLZ (3 doses) } \\
\text { vs. PCB } \\
(\mathrm{N}=165)\end{array}$} & \multirow[t]{2}{*}{$\begin{array}{l}\text { ACR20/ } \\
\text { Week } 16\end{array}$} & $\begin{array}{l}\text { HAQ- } \\
\text { DI }\end{array}$ & $\begin{array}{l}\text { CLZ 200mg: } 1.4(0.7) \\
\text { CLZ 100mg: } 1.3(0.7) \\
\text { CLZ 25mg: } 1.4(0.6) \\
\text { PCB: } 1.4(0.6)\end{array}$ & $\begin{array}{c}\Delta \text { score } \\
\\
\text { Mean [95\% CI] (calculated SD) } \\
\text { CLZ 200mg: }-0.26[-0.42,-0.09] \\
(0.51) \\
\begin{aligned} & \text { CLZ 100mg: }-0.40[-0.56,-0.24] \\
&(0.52) \\
& \text { CLZ 25mg: }-0.44[-0.61,-0.28] \\
&(0.53) \\
& \text { PCB: }-0.27[-0.43,-0.11](0.52)\end{aligned}\end{array}$ & NS & $\begin{array}{c}\text { SRM at Week 16: } \\
\text { CLZ 200mg }=-0.51 \\
\text { CLZ 100mg }=-0.77 \\
\text { CLZ 25mg }=-0.83 \\
\text { PCB }=-0.52\end{array}$ \\
\hline & & & $\begin{array}{l}\text { SF-36 } \\
\text { PCS }\end{array}$ & NA & $\Delta$ score & NS & $\begin{array}{l}\text { SRM at Week 16: } \\
\text { CLZ 200mg = } 0.52\end{array}$ \\
\hline
\end{tabular}




\begin{tabular}{|c|c|c|c|c|c|c|c|}
\hline & & & & & $\begin{array}{c}\text { Mean [95\% CI] (calculated SD) } \\
\text { CLZ 200mg: } 4.1[1.6,6.5](7.81) \\
\text { CLZ 100mg: } 4.6[2.2,7.0](7.84) \\
\text { CLZ 25mg: } 6.5[4.0,8.9](7.91) \\
\text { PCB: } 4.4[2.0,6.8](7.74)\end{array}$ & & $\begin{array}{c}\text { CLZ 100mg }=0.59 \\
\text { CLZ 25mg }=0.82 \\
\text { PCB }=0.57\end{array}$ \\
\hline \multicolumn{8}{|l|}{ Others } \\
\hline $\begin{array}{l}\text { Author / } \\
\text { year/ (study } \\
\text { acronyms) }\end{array}$ & $\begin{array}{l}\text { Intervention/ } \\
\text { comparator } \\
(\text { sample size, } \mathbf{N})\end{array}$ & $\begin{array}{c}\text { Primary } \\
\text { Outcome/ } \\
\text { Time } \\
\text { point }\end{array}$ & PROM & $\begin{array}{c}\text { Baseline PROM } \\
\text { Mean (SD) } \\
\text { (unless specified) }\end{array}$ & $\begin{array}{c}\text { End point PROM/ } \Delta \text { score } \\
\text { Mean (SD) } \\
\text { (unless specified) }\end{array}$ & $\begin{array}{l}\text { P value of } \Delta \\
\text { scores of } \\
\text { interventions } \\
\text { vs. PCB } \\
\text { group }\end{array}$ & $\begin{array}{c}\text { Effect sizes at primary end point } \\
\text { (unless specified) }\end{array}$ \\
\hline \multirow[t]{2}{*}{$\begin{array}{l}\text { Mease, et al. } \\
2018 \\
\text { Phase II }\end{array}$} & \multirow[t]{2}{*}{$\begin{array}{l}\text { ABT-122 } \\
\text { ( } 2 \text { doses) } \\
\text { vs. PCB } \\
\text { vs. ADA } \\
(\mathrm{N}=240)\end{array}$} & \multirow[t]{2}{*}{$\begin{array}{l}\text { ACR20/ } \\
\text { Week } 12\end{array}$} & HAQ-S & $\begin{array}{c}\text { ABT-122 240mg: } 1.3(0.6) \\
\text { ABT-122 120mg: } 1.3(0.6) \\
\text { PCB: } 1.2(0.6) \\
\text { ADA: } 1.3(0.6)\end{array}$ & $\begin{array}{c}\frac{\Delta \text { score }}{2} \\
\text { ABT-122 240mg: }-0.56 \\
\text { ABT-122 120mg: }-0.55 \\
\text { PCB: }-0.28 \\
\text { ADA: }-0.58 \\
\\
\text { Improved HAQ-S } \geq 0.5 \\
\text { ABT-122 240mg: } 54.8 \% \\
\text { ABT-122 120mg: } 43.7 \% \\
\text { PCB: } 25.0 \% \\
\text { ADA: } 41.7 \%\end{array}$ & 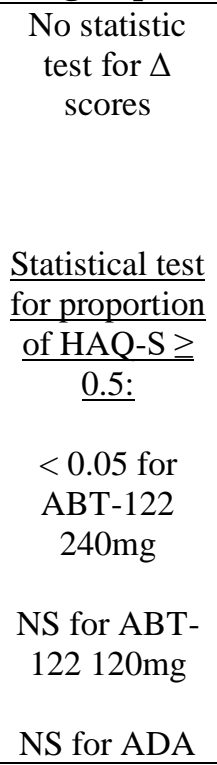 & $\begin{array}{c}\text { ES }_{1} \text { at Week 12: } \\
\text { ABT-122 240mg: }-0.93 \\
\text { ABT-122 120mg: }-0.92 \\
\text { PCB: }-0.47 \\
\text { ADA: }-0.97\end{array}$ \\
\hline & & & $\begin{array}{l}\text { SF-36 } \\
\text { PCS or } \\
\text { PF }\end{array}$ & NA & NA & NA & NA \\
\hline
\end{tabular}

$\mathrm{ES}_{1}$ : Effect size 1 (the mean difference divided by standard deviation of baseline score; $\mathrm{ES}_{2}$ : Effect size 2 (the mean difference divided by the pooled standard deviation, i.e. Cohen's $d$ ); SRM: Standardized response mean (mean difference divided by the standard deviation of the differences between baseline and assessment end point); " $\mathrm{SD}$ of percentage $\Delta ;{ }^{\delta} \mathrm{SRM}$ for improvement, a negative value indicate deterioration; ${ }^{*}$ Effect sizes estimated based on mean and SD of change scores calculated from median and IQR from original publication;* early escape for patients with inadequate response in the PCB group to active treatment group at Week 16; ** option to switch to treatment group at Week 24; $\Delta$ : change. Abbreviations: ACR: American College of Rheumatology Response criteria; ABT: abatacept; ADA: adalimumab; ALC: alefacept; BIW: twice a week; BRO: brodalumab; CI: confidence interval; CLZ: clazakizumab; CZP: certolizumab; ETN: etanercept; EULAR: European League Against Rheumatic Diseases; FIL: filgotinib; GOL: golimumab; GUS: guselkumab; HAQ-DI: Health Assessment Questionnaire - Disability index; IFX: infliximab; IXE: Ixekizumab; ivi: intravenous IQR: interquartile range; LS: least squares; MCID: minimally clinically important difference; MTX: methotrexate; NA: not available; NS: not significant; PsARC: Psoriatic arthritis Response Criteria; PASI: psoriasis area and severity index; PCB: placebo; PCS: physical component summary of SF-36; PF: physical functioning domain of SF-36; QW: once a week; Q2W: once every 2-week; SD: standard deviation; SE: standard error; SEC: secukinumab; SF-36: 


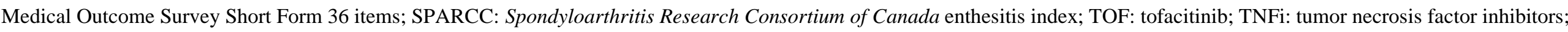
Ustekinumab: UST; Vs.: versus. 
Table A.3. Quality assessment using OMERACT good method checklist for HAQ-DI

\begin{tabular}{|c|c|c|c|c|c|c|}
\hline $1^{\text {st }}$ Author / year/ (study acronyms/ drug) & $\begin{array}{l}\text { Appropriate } \\
\text { time interval }\end{array}$ & $\begin{array}{c}\text { Expected } \\
\text { change in one/ } \\
\text { both groups }\end{array}$ & $\begin{array}{c}\text { Hypothesis of } \\
\text { change stated } \\
\text { a priori } \\
\end{array}$ & $\begin{array}{l}\text { Adequate statistical } \\
\text { methods for } \\
\text { hypothesis testing }\end{array}$ & $\begin{array}{c}\text { Otherwise } \\
\text { good methods }\end{array}$ & $\begin{array}{l}\text { Quality assessment } \\
\text { rating }\end{array}$ \\
\hline Antoni, 2005 (IMPACT) & Green & Green & Amber & Amber & Green & AMBER (+) \\
\hline Antoni, 2005 (IMPACT2) & Green & Green & Green & Amber & Green & AMBER (+) \\
\hline Mease, 2005 (ADEPT) & Green & Green & Green & Green & Green & GREEN (+) \\
\hline Genovese, 2007 (adalimumab) & Green & Green & Amber & Green & Green & GREEN (+) \\
\hline Mease, 2000 (etanercept) & Green & Green & Amber & Amber & Green & AMBER (+) \\
\hline Mease. 2010 (etanercept) & Green & Green & Amber & Green & Green & GREEN (+) \\
\hline Gniadecki, 2012 (PRESTA) & Green & Green & Amber & Green & Green & GREEN (+) \\
\hline Mease. 2019 (SEAM-PsA) & Amber & Green & Amber & Amber & Amber & AMBER (+/-) \\
\hline Kavanaugh, 2009 (GO-REVEAL) & Green & Green & Amber & Green & Green & GREEN (+) \\
\hline Kavanaugh, 2017 (GO-VIBRANT) & Green & Green & Amber & Green & Green & GREEN (+) \\
\hline Gladman, 2014 (RAPID-PsA) & Green & Green & Green & Green & Green & GREEN (+) \\
\hline McInnes, 2014 (secukinumab) & Green & Green & Amber & Amber & Green & AMBER (+) \\
\hline Mease, 2015 (FUTURE I) & Green & Green & Amber & Green & Green & GREEN (+) \\
\hline McInnes, 2015 FUTURE II) & Green & Green & Amber & Green & Green & GREEN (+) \\
\hline Kavanaugh, 2016 (FUTURE II) -subgroup analysis & Green & Green & Amber & Green & Green & GREEN (+) \\
\hline Nash P, 2018 (FUTURE III) & Green & Green & Amber & Green & Green & GREEN (+) \\
\hline Mease, 2017 (SPIRIT-P1) & Green & Green & Amber & Green & Green & GREEN (+) \\
\hline Nash, 2017 (SPIRIT-P2) & Green & Green & Amber & Green & Green & GREEN (+) \\
\hline Mease, 2014 (brodalumab) & Green & Green & Amber & Amber & Green & AMBER (+) \\
\hline Gottlieb, 2009 (ustekinumab) & Green & Green & Amber & Amber & Green & AMBER (+) \\
\hline McInnes, 2013 (PSUMMIT I) & Green & Green & Amber & Amber & Green & AMBER (+) \\
\hline Ritchlin, 2014 (PSUMMIT II) & Green & Green & Amber & Amber & Green & AMBER (+) \\
\hline Araugo, 2019 (ECLIPSA) & Green & Green & Amber & Amber & Green & AMBER (+) \\
\hline Deodhar, 2018 (guselkumab) & Green & Green & Amber & Green & Green & GREEN (+) \\
\hline Mease, 2011 (abatacept) & Green & Green & Amber & Red & Green & RED (+/-) \\
\hline Mease, 2017 (ASTRAEA) & Green & Green & Amber & Green & Green & GREEN (+) \\
\hline Mease, 2006 (alefacept) & Green & Green & Amber & Red & Amber & RED (+/-) \\
\hline Mease, 2017 (OPAL Broaden) & Green & Green & Green & Green & Green & GREEN (+) \\
\hline Gladman, 2017 (OPAL Beyond) & Green & Green & Green & Green & Green & GREEN (+) \\
\hline Mease, 2018 (EQUATOR) & Green & Green & Amber & Green & Green & GREEN (+) \\
\hline Mease, 2016 (clazakizumab) & Green & Green & Amber & Green & Green & GREEN (+) \\
\hline
\end{tabular}


Table A.4. Quality assessment using OMERACT good method checklist for HAQ-S

\begin{tabular}{|c|c|c|c|c|c|c|}
\hline $1^{\text {st }}$ Author / year/ (study acronyms/ drug) & $\begin{array}{c}\text { Appropriate } \\
\text { time interval }\end{array}$ & $\begin{array}{c}\text { Expected } \\
\text { change in one/ } \\
\text { both groups }\end{array}$ & $\begin{array}{l}\text { Hypothesis of } \\
\text { change stated a } \\
\text { priori }\end{array}$ & $\begin{array}{c}\text { Adequate } \\
\text { statistical } \\
\text { methods for } \\
\text { hypothesis } \\
\text { testing }\end{array}$ & $\begin{array}{l}\text { Otherwise good } \\
\text { methods }\end{array}$ & $\begin{array}{c}\text { Quality } \\
\text { assessment } \\
\text { rating }\end{array}$ \\
\hline Mease, 2018 (ABT-122) & Green & Green & Amber & Green & Amber & AMBER (+) \\
\hline
\end{tabular}


Table A.5. Quality assessment using OMERACT good method checklist for SF-36 PCS

\begin{tabular}{|c|c|c|c|c|c|c|}
\hline $1^{\text {st }}$ Author / year/ (study acronyms/ drug) & $\begin{array}{l}\text { Appropriate } \\
\text { time interval }\end{array}$ & $\begin{array}{c}\text { Expected } \\
\text { change in one/ } \\
\text { both groups }\end{array}$ & $\begin{array}{l}\text { Hypothesis of } \\
\text { change stated } \\
\text { a priori }\end{array}$ & $\begin{array}{l}\text { Adequate statistical } \\
\text { methods for } \\
\text { hypothesis testing }\end{array}$ & $\begin{array}{l}\text { Otherwise good } \\
\text { methods }\end{array}$ & $\begin{array}{c}\text { Quality } \\
\text { assessment } \\
\text { rating } \\
\end{array}$ \\
\hline Antoni, 2005 (IMPACT2) & Green & Green & Green & Amber & Green & AMBER (+) \\
\hline Mease, 2005 (ADEPT) & Green & Green & Green & Green & Green & GREEN (+) \\
\hline Genovese, 2007 (adalimumab) & Green & Green & Green & Green & Green & GREEN (+) \\
\hline Mease, 2000 (etanercept) & Green & Green & Amber & Amber & Green & AMBER (+) \\
\hline Mease. 2010 (etanercept) & Green & Green & Amber & Green & Green & GREEN (+) \\
\hline Mease. 2019 (SEAM-PsA) & Amber & Green & Amber & Green & Amber & AMBER (+) \\
\hline Kavanaugh, 2009 (GO-REVEAL) & Green & Green & Amber & Green & Green & GREEN (+) \\
\hline Kavanaugh, 2017 (GO-VIBRANT) & Green & Green & Amber & Green & Green & GREEN (+) \\
\hline Gladman, 2014 (RAPID-PsA) & Green & Green & Green & Green & Green & GREEN (+) \\
\hline McInnes, 2014 (secukinumab) & Green & Green & Amber & Green & Green & GREEN (+) \\
\hline Mease, 2015 (FUTURE I) & Green & Green & Amber & Green & Green & GREEN (+) \\
\hline McInnes, 2015 FUTURE II) & Green & Green & Amber & Green & Green & GREEN (+) \\
\hline Kavanaugh, 2016 (FUTURE II) -subgroup analysis & Green & Green & Amber & Green & Green & GREEN (+) \\
\hline Nash P, 2018 (FUTURE III) & Green & Green & Amber & Green & Green & GREEN (+) \\
\hline Mease, 2017 (SPIRIT-P1) & Green & Green & Amber & Green & Green & GREEN (+) \\
\hline Nash, 2017 (SPIRIT-P2) & Green & Green & Amber & Green & Green & GREEN (+) \\
\hline Mease, 2014 (brodalumab) & Green & Green & Amber & Red & Amber & RED (+) \\
\hline McInnes, 2013 (PSUMMIT I) & Green & Green & Amber & Amber & Green & AMBER (+) \\
\hline Ritchlin, 2014 (PSUMMIT II) & Green & Green & Amber & Amber & Green & AMBER (+) \\
\hline Araugo, 2019 (ECLIPSA) & Green & Green & Amber & Amber & Green & AMBER (+) \\
\hline Deodhar, 2018 (guselkumab) & Green & Green & Amber & Green & Green & GREEN (+) \\
\hline Mease, 2011 (abatacept) & Green & Green & Amber & Green & Amber & AMBER (+) \\
\hline Mease, 2017 (ASTRAEA) & Green & Green & Amber & Green & Amber & AMBER (+) \\
\hline Strand, 2019 (OPAL Broaden) & Green & Green & Green & Green & Green & GREEN (+) \\
\hline Strand, 2019 (OPAL Beyond) & Green & Green & Green & Green & Green & GREEN (+) \\
\hline Mease, 2016 (clazakizumab) & Green & Green & Amber & Green & Green & GREEN (+) \\
\hline
\end{tabular}


Table A.6. Quality assessment using OMERACT good method checklist for SF-36 PF

\begin{tabular}{|c|c|c|c|c|c|c|}
\hline $1^{\text {st }}$ Author / year/ (study acronyms/ drug) & $\begin{array}{l}\text { Appropriate time } \\
\text { interval }\end{array}$ & $\begin{array}{l}\text { Expected change in } \\
\text { one/ both groups }\end{array}$ & $\begin{array}{l}\text { Hypothesis of } \\
\text { change stated a } \\
\text { priori } \\
\end{array}$ & $\begin{array}{l}\text { Adequate statistical } \\
\text { methods for hypothesis } \\
\text { testing }\end{array}$ & $\begin{array}{l}\text { Otherwise good } \\
\text { methods }\end{array}$ & $\begin{array}{c}\text { Quality } \\
\text { assessment } \\
\text { rating } \\
\end{array}$ \\
\hline Kavanaugh, 2006 (IMPACT2) & Green & Amber & Amber & Red & Amber & RED (+) \\
\hline Mease, 2017 (OPAL Broaden) & Green & Green & Amber & Green & Amber & AMBER (+) \\
\hline Gladman, 2017 (OPAL Beyond) & Green & Green & Amber & Green & Amber & AMBER (+) \\
\hline Mease, 2018 (EQUATOR) & Green & Green & Amber & Red & Amber & RED (+) \\
\hline
\end{tabular}

